\title{
A convergent evolving finite element algorithm for mean curvature flow of closed surfaces
}

\author{
Balázs Kovács · Buyang Li • \\ Christian Lubich
}

This paper is dedicated to Gerhard Dziuk on the occasion of his 70th birthday and to Gerhard Huisken on the occasion of his 60th birthday.

\begin{abstract}
A proof of convergence is given for semi- and full discretizations of mean curvature flow of closed two-dimensional surfaces. The numerical method proposed and studied here combines evolving finite elements, whose nodes determine the discrete surface like in Dziuk's method, and linearly implicit backward difference formulae for time integration. The proposed method differs from Dziuk's approach in that it discretizes Huisken's evolution equations for the normal vector and mean curvature and uses these evolving geometric quantities in the velocity law projected to the finite element space. This numerical method admits a convergence analysis in the case of finite elements of polynomial degree at least two and backward difference formulae of orders two to five. The error analysis combines stability estimates and consistency estimates to yield optimal-order $H^{1}$-norm error bounds for the computed surface position, velocity, normal vector and mean curvature. The stability analysis is based on the matrix-vector formulation of the finite element method and does not use geometric arguments. The geometry enters only into the consistency estimates. Numerical experiments illustrate and complement the theoretical results.
\end{abstract}

Keywords mean curvature flow $\cdot$ geometric evolution equations · evolving surface finite elements - linearly implicit backward difference formula . stability $\cdot$ convergence analysis

Mathematics Subject Classification (2000) 35R01 - 65M60 - 65M15 • $65 \mathrm{M} 12$

B. Kovács and Ch. Lubich

Mathematisches Institut, Universität Tübingen,

Auf der Morgenstelle 10, 72076 Tübingen, Germany

E-mail: \{kovacs,lubich\}@na.uni-tuebingen.de

B. Li

Department of Applied Mathematics, Hong Kong Polytechnic University,

Kowloon, Hong Kong

E-mail: buyang.li@polyu.edu.hk 


\section{Introduction}

Mean curvature flow is a geometric evolution equation that has been studied intensively in geometric analysis in the last decades, as is evidenced by the recent monographs by Ecker [25] and Mantegazza [37] and the numerous references therein, going back to the pioneering work by Brakke [9] and Huisken [30]. As White [42] puts it succinctly in his review article: "There are many processes by which a curve or surface can evolve, but among them one is arguably the most natural: the mean curvature flow."

Approximating the mean curvature flow by numerical methods was first addressed by Dziuk [19] in 1990. He proposed a finite element method based on a weak formulation of the mean curvature flow as a (formally) heat-like partial differential equation, in which the moving nodes of the finite element mesh determine the approximate evolving surface. However, proving convergence of Dziuk's method or of other evolving finite element methods, such as the method proposed by Barrett, Garcke \& Nürnberg [5], has remained an open problem for the mean curvature flow of closed two-dimensional surfaces (or higher-dimensional hypersurfaces).

Convergence of an evolving finite element method was shown for the mean curvature flow of closed curves (known as curve-shortening flow) in [20,14]; see also [3]. For a non-evolving finite element discretization of the nonlinear parabolic equation describing the mean curvature flow of a two-dimensional surface that is a graph (with the evolving height over a fixed domain as the unknown variable), convergence of optimal order was shown in [13,15]. To our knowledge, the only convergence result of a numerical method for mean curvature flow of closed surfaces in the literature is for a level set method [12], under very weak regularity assumptions but consequently with very low order of convergence and only under very restrictive conditions between the mesh size and the regularization parameter that appears in the method. We further refer to Deckelnick, Dziuk \& Elliott [16] for an excellent review of numerical approaches to mean curvature flow and related problems, including applications in various fields of science.

In the present paper we formulate a novel evolving finite element method for mean curvature flow of closed two-dimensional surfaces and prove optimalorder convergence over time intervals on which the evolving surface remains sufficiently regular. We study stability and convergence for both the finite element semi-discretization and the full discretization obtained with a linearly implicit backward difference time discretization. Our approach shares with Dziuk's method the property that the moving nodes of a finite element mesh determine the approximate evolving surface. However, the method presented here discretizes equations that are different from the equation discretized by Dziuk. In his approach, a weak formulation of the quasi-heat equation describing mean curvature flow is discretized, whereas in the present work evolution equations for the normal vector and the mean curvature are discretized, which then yield the velocity of the surface evolving under mean curvature flow. Evolution equations for geometric quantities on a surface evolving under mean 
curvature flow have been an important tool in the analysis of mean curvature flow ever since Huisken's 1984 paper [30], but apparently they have so far not been used in the numerical approximation of mean curvature flow.

The numerical method based on the discretization of evolution equations of geometric quantities, as presented here, is computationally more expensive than Dziuk's method (roughly by about a factor 2), but on the other hand it provides full-order approximations to basic geometric quantities — the normal vector and curvature - in addition to the position and velocity of the surface.

Our numerical approach is related to our previous paper [33], where we study the convergence of finite elements on an evolving surface driven by diffusion on the surface. The convergence analysis of the present paper relies on techniques developed in that previous paper. As in [33], the stability analysis works with the matrix-vector formulation of the method and does not use geometric arguments. The geometry only enters into the analysis of the consistency error.

The paper is organized as follows:

In Section 2 we recall Huisken's evolution equations for the normal vector and mean curvature of surfaces under mean curvature flow and formulate the system of equations that will be discretized in the following.

In Section 3 we formulate the semi-discretization in space by the evolving surface finite element method, and in Section 4 we state and discuss the convergence result for the semi-discretization (Theorem 4.1).

In Section 5 we formulate the full discretization with linearly implicit backward difference formulae, and in Section 6 we state the convergence result for the full discretization (Theorem 6.1).

In Sections 7 to 12 we give the proofs of the convergence results. Theorem 4.1 is proved in Sections 7, 8 and 9, where we study stability, consistency and their combination, respectively, for the semi-discretization. Theorem 6.1 is proved in Sections 10, 11 and 12, where again we study stability, consistency and their combination, respectively, this time for the full discretization.

In Section 13 we present results of some numerical experiments.

Finally, Section 14 collects some conclusions and adds comments on related further topics that are not addressed in the main text.

We use the notational convention to denote vectors in $\mathbb{R}^{3}$ by italic letters, but to denote finite element nodal vectors in $\mathbb{R}^{N}$ and $\mathbb{R}^{3 N}$ by boldface lowercase letters and finite element mass and stiffness matrices by boldface capitals. All boldface symbols in this paper will thus be related to the matrix-vector formulation of the finite element method. 


\section{Evolution equations for mean curvature flow}

2.1 Basic notions and notation

We consider the evolving two-dimensional closed surface $\Gamma(t) \subset \mathbb{R}^{3}$ as the image

$$
\Gamma(t)=\left\{X(p, t): p \in \Gamma^{0}\right\}
$$

of a smooth mapping $X: \Gamma^{0} \times[0, T] \rightarrow \mathbb{R}^{3}$ such that $X(\cdot, t)$ is an embedding for every $t$. Here, $\Gamma^{0}$ is a smooth closed initial surface, and $X(p, 0)=p$. In view of the subsequent numerical discretization, it is convenient to think of $X(p, t)$ as the position at time $t$ of a moving particle with label $p$, and of $\Gamma(t)$ as a collection of such particles. To indicate the dependence of the surface on $X$, we will write

$$
\Gamma(t)=\Gamma[X(\cdot, t)], \quad \text { or briefly } \quad \Gamma[X]
$$

when the time $t$ is clear from the context. The velocity $v(x, t) \in \mathbb{R}^{3}$ at a point $x=X(p, t) \in \Gamma(t)$ equals

$$
\partial_{t} X(p, t)=v(X(p, t), t)
$$

For a known velocity field $v$, the position $X(p, t)$ at time $t$ of the particle with label $p$ is obtained by solving the ordinary differential equation (2.1) from 0 to $t$ for a fixed $p$.

For a function $u(x, t)(x \in \Gamma(t), 0 \leq t \leq T)$ we denote the material derivative (with respect to the parametrization $X$ ) as

$$
\partial^{\bullet} u(x, t)=\frac{\mathrm{d}}{\mathrm{d} t} u(X(p, t), t) \quad \text { for } \quad x=X(p, t) .
$$

On any regular surface $\Gamma \subset \mathbb{R}^{3}$, we denote by $\nabla_{\Gamma} u: \Gamma \rightarrow \mathbb{R}^{3}$ the tangential gradient of a function $u: \Gamma \rightarrow \mathbb{R}$, and in the case of a vector-valued function $u=\left(u_{1}, u_{2}, u_{3}\right)^{T}: \Gamma \rightarrow \mathbb{R}^{3}$, we let $\nabla_{\Gamma} u=\left(\nabla_{\Gamma} u_{1}, \nabla_{\Gamma} u_{2}, \nabla_{\Gamma} u_{3}\right)$. We thus use the convention that the gradient of $u$ has the gradient of the components as column vectors. We denote by $\nabla_{\Gamma} \cdot f$ the surface divergence of a vector field $f$ on $\Gamma$, and by $\Delta_{\Gamma} u=\nabla_{\Gamma} \cdot \nabla_{\Gamma} u$ the Laplace-Beltrami operator applied to $u$; see the review [16] or [25, Appendix A] or any textbook on differential geometry for these notions.

We denote the unit outer normal vector field to $\Gamma$ by $\nu: \Gamma \rightarrow \mathbb{R}^{3}$. Its surface gradient contains the (extrinsic) curvature data of the surface $\Gamma$. At every $x \in \Gamma$, the matrix of the extended Weingarten map,

$$
A(x)=\nabla_{\Gamma} \nu(x),
$$

is a symmetric $3 \times 3$ matrix (see, e.g., [41, Proposition 20]). Apart from the eigenvalue 0 with eigenvector $\nu(x)$, its other two eigenvalues are the principal curvatures $\kappa_{1}$ and $\kappa_{2}$ at the point $x$ on the surface. They determine the fundamental quantities

$$
H:=\operatorname{tr}(A)=\kappa_{1}+\kappa_{2}, \quad|A|^{2}=\kappa_{1}^{2}+\kappa_{2}^{2},
$$


where $|A|$ denotes the Frobenius norm of the matrix $A$. Here, $H$ is called the mean curvature (as in most of the literature, we do not put a factor $1 / 2$ ).

2.2 Evolution equations for normal vector and mean curvature of a surface moving under mean curvature flow

Mean curvature flow sets the velocity $(2.1)$ of the surface $\Gamma[X]$ to

$$
v=-H \nu \text {. }
$$

The geometric quantities on the right-hand side are known to satisfy the following evolution equations.

Lemma 2.1 (Huisken [30]) For a regular surface $\Gamma[X]$ moving under mean curvature flow, the normal vector and the mean curvature satisfy

$$
\begin{aligned}
\partial^{\bullet} \nu & =\Delta_{\Gamma[X]} \nu+|A|^{2} \nu, \\
\partial^{\bullet} H & =\Delta_{\Gamma[X]} H+|A|^{2} H .
\end{aligned}
$$

Proof The evolution equation for $H$ is given in [30, Corollary 3.5]. In [30, Lemma 3.3], the following evolution equation for the normal vector is derived:

$$
\partial^{\bullet} \nu=\nabla_{\Gamma[X]} H .
$$

On any surface $\Gamma$, it holds true that (see [25, (A.9)] or [41, Proposition 24])

$$
\nabla_{\Gamma[X]} H=\Delta_{\Gamma[X]} \nu+|A|^{2} \nu
$$

which then gives the stated evolution equation for $\nu$; see also [25, (B.11)].

\subsection{The system of equations used for discretization}

Collecting the above equations, we have reformulated mean curvature flow as the system of semilinear parabolic equations (2.4)-(2.5) on the surface coupled to the velocity law (2.3). The numerical discretization is based on a weak formulation of (2.3)-(2.5): we use the velocity equation (2.1) together with

$$
\begin{aligned}
\int_{\Gamma[X]} \nabla_{\Gamma[X]} v & \nabla_{\Gamma[X]} \varphi^{v}+\int_{\Gamma[X]} v \cdot \varphi^{v} \\
= & -\int_{\Gamma[X]} \nabla_{\Gamma[X]}(H \nu) \cdot \nabla_{\Gamma[X]} \varphi^{v}-\int_{\Gamma[X]} H \nu \cdot \varphi^{v} \\
\int_{\Gamma[X]} \partial^{\bullet} \nu \cdot \varphi^{\nu} & +\int_{\Gamma[X]} \nabla_{\Gamma[X]} \nu \cdot \nabla_{\Gamma[X]} \varphi^{\nu}=\int_{\Gamma[X]}\left|\nabla_{\Gamma[X]} \nu\right|^{2} \nu \cdot \varphi^{\nu} \\
\int_{\Gamma[X]} \partial^{\bullet} H \varphi^{H} & +\int_{\Gamma[X]} \nabla_{\Gamma[X]} H \cdot \nabla_{\Gamma[X]} \varphi^{H}=\int_{\Gamma[X]}\left|\nabla_{\Gamma[X]} \nu\right|^{2} H \varphi^{H}
\end{aligned}
$$


for all test functions $\varphi^{v} \in H^{1}(\Gamma[X])^{3}$ and $\varphi^{\nu} \in H^{1}(\Gamma[X])^{3}, \varphi^{H} \in H^{1}(\Gamma[X])$. Here, we use the Sobolev space $H^{1}(\Gamma)=\left\{u \in L^{2}(\Gamma): \nabla_{\Gamma} u \in L^{2}(\Gamma)\right\}$. This system is complemented with the initial data $X^{0}, \nu^{0}$ and $H^{0}$.

Throughout the paper both the usual Euclidean scalar product for vectors and the Frobenius inner product for matrices (which equals to the Euclidean product using an arbitrary vectorisation) are denoted by a dot.

It is instructive to compare this system with the equations on which Dziuk's discretization in [19] is based. There, he uses that $-H \nu=\Delta_{\Gamma} x_{\Gamma}$, with $x_{\Gamma}$ denoting the identity map on $\Gamma$, and employs the velocity equation (2.1) together with the weak formulation of the velocity law $v=\Delta_{\Gamma[X]} x_{\Gamma[X]}$ :

$$
\int_{\Gamma[X]} v \cdot \varphi=-\int_{\Gamma[X]} \nabla_{\Gamma[X]} x_{\Gamma[X]} \cdot \nabla_{\Gamma[X]} \varphi
$$

for all test functions $\varphi \in H^{1}(\Gamma[X])^{3}$. This weak formulation clearly has the charm of greater simplicity than (2.6), but no convergence proof for a discretization based on this formulation is known to date.

\section{Evolving finite element semi-discretization}

\subsection{Evolving surface finite elements}

We formulate the evolving surface finite element (ESFEM) discretization for the velocity law coupled with evolution equations on the evolving surface, following the description in [33], which is based on [18] and [17]. We use simplicial finite elements and continuous piecewise polynomial basis functions of degree $k$, as defined in [17, Section 2.5].

We triangulate the given smooth initial surface $\Gamma^{0}$ by an admissible family of triangulations $\mathcal{T}_{h}$ of decreasing maximal element diameter $h$; see [21] for the notion of an admissible triangulation, which includes quasi-uniformity and shape regularity. For a momentarily fixed $h$, we denote by $\mathbf{x}^{0}$ the vector in $\mathbb{R}^{3 N}$ that collects all nodes $p_{j}(j=1, \ldots, N)$ of the initial triangulation. By piecewise polynomial interpolation of degree $k$, the nodal vector defines an approximate surface $\Gamma_{h}^{0}$ that interpolates $\Gamma^{0}$ in the nodes $p_{j}$. We will evolve the $j$ th node in time, denoted $x_{j}(t)$ with $x_{j}(0)=p_{j}$, and collect the nodes at time $t$ in a column vector

$$
\mathbf{x}(t) \in \mathbb{R}^{3 N} .
$$

We just write $\mathbf{x}$ for $\mathbf{x}(t)$ when the dependence on $t$ is not important.

By piecewise polynomial interpolation on the plane reference triangle that corresponds to every curved triangle of the triangulation, the nodal vector $\mathbf{x}$ defines a closed surface denoted by $\Gamma_{h}[\mathbf{x}]$. We can then define globally continuous finite element basis functions

$$
\phi_{i}[\mathbf{x}]: \Gamma_{h}[\mathbf{x}] \rightarrow \mathbb{R}, \quad i=1, \ldots, N,
$$


which have the property that on every triangle their pullback to the reference triangle is polynomial of degree $k$, and which satisfy at the node $x_{j}$

$$
\phi_{i}[\mathbf{x}]\left(x_{j}\right)=\delta_{i j} \quad \text { for all } i, j=1, \ldots, N
$$

These functions span the finite element space on $\Gamma_{h}[\mathbf{x}]$,

$$
S_{h}[\mathbf{x}]=S_{h}\left(\Gamma_{h}[\mathbf{x}]\right)=\operatorname{span}\left\{\phi_{1}[\mathbf{x}], \phi_{2}[\mathbf{x}], \ldots, \phi_{N}[\mathbf{x}]\right\}
$$

For a finite element function $u_{h} \in S_{h}[\mathbf{x}]$, the tangential gradient $\nabla_{\Gamma_{h}[\mathbf{x}]} u_{h}$ is defined piecewise on each element.

The discrete surface at time $t$ is parametrized by the initial discrete surface via the map $X_{h}(\cdot, t): \Gamma_{h}^{0} \rightarrow \Gamma_{h}[\mathbf{x}(t)]$ defined by

$$
X_{h}\left(p_{h}, t\right)=\sum_{j=1}^{N} x_{j}(t) \phi_{j}[\mathbf{x}(0)]\left(p_{h}\right), \quad p_{h} \in \Gamma_{h}^{0},
$$

which has the properties that $X_{h}\left(p_{j}, t\right)=x_{j}(t)$ for $j=1, \ldots, N$, that $X_{h}\left(p_{h}, 0\right)$ $=p_{h}$ for all $p_{h} \in \Gamma_{h}^{0}$, and

$$
\Gamma_{h}[\mathbf{x}(t)]=\Gamma\left[X_{h}(\cdot, t)\right]
$$

where the right-hand side equals $\left\{X_{h}\left(p_{h}, t\right): p_{h} \in \Gamma_{h}^{0}\right\}$ like in Section 2.1.

The discrete velocity $v_{h}(x, t) \in \mathbb{R}^{3}$ at a point $x=X_{h}\left(p_{h}, t\right) \in \Gamma\left[X_{h}(\cdot, t)\right]$ is given by

$$
\partial_{t} X_{h}\left(p_{h}, t\right)=v_{h}\left(X_{h}\left(p_{h}, t\right), t\right) .
$$

In view of the transport property of the basis functions [21],

$$
\frac{\mathrm{d}}{\mathrm{d} t}\left(\phi_{j}[\mathbf{x}(t)]\left(X_{h}\left(p_{h}, t\right)\right)\right)=0
$$

the discrete velocity equals, for $x \in \Gamma_{h}[\mathbf{x}(t)]$,

$$
v_{h}(x, t)=\sum_{j=1}^{N} v_{j}(t) \phi_{j}[\mathbf{x}(t)](x) \quad \text { with } v_{j}(t)=\dot{x}_{j}(t),
$$

where the dot denotes the time derivative $\mathrm{d} / \mathrm{d} t$. Hence, the discrete velocity $v_{h}(\cdot, t)$ is in the finite element space $S_{h}[\mathbf{x}(t)]$, with nodal vector $\mathbf{v}(t)=\dot{\mathbf{x}}(t)$.

The discrete material derivative of a finite element function $u_{h}(x, t)$ with nodal values $u_{j}(t)$ is

$$
\partial_{h}^{\bullet} u_{h}(x, t)=\frac{\mathrm{d}}{\mathrm{d} t} u_{h}\left(X_{h}\left(p_{h}, t\right)\right)=\sum_{j=1}^{N} \dot{u}_{j}(t) \phi_{j}[\mathbf{x}(t)](x) \quad \text { at } \quad x=X_{h}\left(p_{h}, t\right) .
$$


3.2 ESFEM spatial semi-discretization

The finite element spatial semi-discretization of the weak coupled parabolic system (2.6) reads as follows: Find the unknown nodal vector $\mathbf{x}(t) \in \mathbb{R}^{3 N}$ and the unknown finite element functions $v_{h}(\cdot, t) \in S_{h}[\mathbf{x}(t)]^{3}$ and $\nu_{h}(\cdot, t) \in$ $S_{h}[\mathbf{x}(t)]^{3}, H_{h}(\cdot, t) \in S_{h}[\mathbf{x}(t)]$ such that

$$
\begin{gathered}
\int_{\Gamma_{h}[\mathbf{x}]} \nabla_{\Gamma_{h}[\mathbf{x}]} v_{h} \cdot \nabla_{\Gamma_{h}[\mathbf{x}]} \varphi_{h}^{v}+\int_{\Gamma_{h}[\mathbf{x}]} v_{h} \cdot \varphi_{h}^{v} \\
=-\int_{\Gamma_{h}[\mathbf{x}]} \nabla_{\Gamma_{h}[\mathbf{x}]}\left(H_{h} \nu_{h}\right) \cdot \nabla_{\Gamma_{h}[\mathbf{x}]} \varphi_{h}^{v}-\int_{\Gamma_{h}[\mathbf{x}]} H_{h} \nu_{h} \cdot \varphi_{h}^{v} \\
\int_{\Gamma_{h}[\mathbf{x}]} \partial_{h}^{\bullet} \nu_{h} \cdot \varphi_{h}^{\nu}+\int_{\Gamma_{h}[\mathbf{x}]} \nabla_{\Gamma_{h}[\mathbf{x}]} \nu_{h} \cdot \nabla_{\Gamma_{h}[\mathbf{x}]} \varphi_{h}^{\nu}=\int_{\Gamma_{h}[\mathbf{x}]}\left|\nabla_{\Gamma_{h}[\mathbf{x}]} \nu_{h}\right|^{2} \nu_{h} \cdot \varphi_{h}^{\nu} \\
\int_{\Gamma_{h}[\mathbf{x}]} \partial_{h}^{\bullet} H_{h} \varphi_{h}^{H}+\int_{\Gamma_{h}[\mathbf{x}]} \nabla_{\Gamma_{h}[\mathbf{x}]} H_{h} \cdot \nabla_{\Gamma_{h}[\mathbf{x}]} \varphi_{h}^{H}=\int_{\Gamma_{h}[\mathbf{x}]}\left|\nabla_{\Gamma_{h}[\mathbf{x}]} \nu_{h}\right|^{2} H_{h} \varphi_{h}^{H}
\end{gathered}
$$

for all $\varphi_{h}^{v} \in S_{h}[\mathbf{x}(t)]^{3}, \varphi_{h}^{\nu} \in S_{h}[\mathbf{x}(t)]^{3}$, and $\varphi_{h}^{H} \in S_{h}[\mathbf{x}(t)]$, with the surface $\Gamma_{h}[\mathbf{x}(t)]=\Gamma\left[X_{h}(\cdot, t)\right]$ given by the differential equation

$$
\partial_{t} X_{h}\left(p_{h}, t\right)=v_{h}\left(X_{h}\left(p_{h}, t\right), t\right), \quad p_{h} \in \Gamma_{h}^{0} .
$$

The initial values for the nodal vector $\mathbf{x}$ are taken as the positions of the nodes of the triangulation of the given initial surface $\Gamma^{0}$. The initial data for $\nu_{h}$ and $H_{h}$ are determined by Lagrange interpolation of $\nu^{0}$ and $H^{0}$, respectively.

In the above approach, the discretization of the evolution equations for $\nu$ and $H$ is done in the usual way of evolving surface finite elements. The velocity law (2.3) is enforced by a Ritz projection of $-H_{h} \nu_{h}$ to the finite element space on $\Gamma_{h}[\mathbf{x}]$. Taking instead just the finite element interpolation of $-H_{h} \nu_{h}$ does not appear sufficient for a convergence analysis. Note that the finite element functions $\nu_{h}$ and $H_{h}$ are not the normal vector and the mean curvature of the discrete surface $\Gamma_{h}[\mathbf{x}(t)]$.

For comparison, Dziuk's discretization [19] uses the differential equation (3.2) together with the ESFEM discretization of (2.7): for all $\varphi_{h} \in S_{h}[\mathbf{x}(t)]^{3}$,

$$
\int_{\Gamma_{h}[\mathbf{x}]} v_{h} \cdot \varphi_{h}=-\int_{\Gamma_{h}[\mathbf{x}]} \nabla_{\Gamma_{h}[\mathbf{x}]} x_{\Gamma_{h}[\mathbf{x}]} \cdot \nabla_{\Gamma_{h}[\mathbf{x}]} \varphi_{h}
$$

\subsection{Matrix-vector formulation}

We collect the nodal values in column vectors $\mathbf{v}=\left(v_{j}\right) \in \mathbb{R}^{3 N}, \mathbf{H}=\left(H_{j}\right) \in \mathbb{R}^{N}$ and $\mathbf{n}=\left(\nu_{j}\right) \in \mathbb{R}^{3 N}$, and denote

$$
\mathbf{u}=\left(\begin{array}{c}
\mathbf{n} \\
\mathbf{H}
\end{array}\right) \in \mathbb{R}^{4 N}
$$


We define the surface-dependent mass matrix $\mathbf{M}(\mathbf{x})$ and stiffness matrix $\mathbf{A}(\mathbf{x})$ on the surface determined by the nodal vector $\mathbf{x}$ :

$$
\begin{aligned}
\left.\mathbf{M}(\mathbf{x})\right|_{i j} & =\int_{\Gamma_{h}[\mathbf{x}]} \phi_{i}[\mathbf{x}] \phi_{j}[\mathbf{x}], \\
\left.\mathbf{A}(\mathbf{x})\right|_{i j} & =\int_{\Gamma_{h}[\mathbf{x}]} \nabla_{\Gamma_{h}[\mathbf{x}]} \phi_{i}[\mathbf{x}] \cdot \nabla_{\Gamma_{h}[\mathbf{x}]} \phi_{j}[\mathbf{x}],
\end{aligned} \quad i, j=1, \ldots, N,
$$

with the finite element nodal basis functions $\phi_{j}[\mathbf{x}] \in S_{h}[\mathbf{x}]$. We define the nonlinear functions $\mathbf{f}(\mathbf{x}, \mathbf{u}) \in \mathbb{R}^{4 N}$ and $\mathbf{g}(\mathbf{x}, \mathbf{u}) \in \mathbb{R}^{3 N}$ by

$$
\mathbf{f}(\mathbf{x}, \mathbf{u})=\left(\begin{array}{l}
\mathbf{f}_{1}(\mathbf{x}, \mathbf{u}) \\
\mathbf{f}_{2}(\mathbf{x}, \mathbf{u})
\end{array}\right)
$$

with $\mathbf{f}_{1}(\mathbf{x}, \mathbf{u}) \in \mathbb{R}^{3 N}$ and $\mathbf{f}_{2}(\mathbf{x}, \mathbf{u}) \in \mathbb{R}^{N}$, given by

$$
\begin{aligned}
\left.\mathbf{f}_{1}(\mathbf{x}, \mathbf{u})\right|_{j+(\ell-1) N} & =\int_{\Gamma_{h}[\mathbf{x}]} \alpha_{h}^{2}\left(\nu_{h}\right)_{\ell} \phi_{j}[\mathbf{x}], \quad \text { with } \quad \alpha_{h}^{2}=\left|\nabla_{\Gamma_{h}[\mathbf{x}]} \nu_{h}\right|^{2}, \\
\left.\mathbf{f}_{2}(\mathbf{x}, \mathbf{u})\right|_{j} & =\int_{\Gamma_{h}[\mathbf{x}]} \alpha_{h}^{2} H_{h} \phi_{j}[\mathbf{x}], \\
\left.\mathbf{g}(\mathbf{x}, \mathbf{u})\right|_{j+(\ell-1) N} & =-\int_{\Gamma_{h}[\mathbf{x}]} H_{h}\left(\nu_{h}\right)_{\ell} \phi_{j}[\mathbf{x}]-\int_{\Gamma_{h}[\mathbf{x}]} \nabla_{\Gamma_{h}[\mathbf{x}]}\left(H_{h}\left(\nu_{h}\right)_{\ell}\right) \cdot \nabla_{\Gamma_{h}[\mathbf{x}]} \phi_{j}[\mathbf{x}],
\end{aligned}
$$

for $j=1, \ldots, N$, and $\ell=1,2,3$.

We further let, for $d=3$ or 4 (with the identity matrices $I_{d} \in \mathbb{R}^{d \times d}$ )

$\mathbf{M}^{[d]}(\mathbf{x})=I_{d} \otimes \mathbf{M}(\mathbf{x}), \quad \mathbf{A}^{[d]}(\mathbf{x})=I_{d} \otimes \mathbf{A}(\mathbf{x}), \quad \mathbf{K}^{[d]}(\mathbf{x})=I_{d} \otimes(\mathbf{M}(\mathbf{x})+\mathbf{A}(\mathbf{x}))$.

When no confusion can arise, we often write $\mathbf{M}(\mathbf{x})$ for $\mathbf{M}^{[d]}(\mathbf{x}), \mathbf{A}(\mathbf{x})$ for $\mathbf{A}^{[d]}(\mathbf{x})$, and $\mathbf{K}(\mathbf{x})$ for $\mathbf{K}^{[d]}(\mathbf{x})$. form:

Then, the equations (3.1) can be written in the following matrix-vector

$$
\begin{aligned}
\mathbf{K}^{[3]}(\mathbf{x}) \mathbf{v} & =\mathbf{g}(\mathbf{x}, \mathbf{u}), \\
\mathbf{M}^{[4]}(\mathbf{x}) \dot{\mathbf{u}}+\mathbf{A}^{[4]}(\mathbf{x}) \mathbf{u} & =\mathbf{f}(\mathbf{x}, \mathbf{u}),
\end{aligned}
$$

and (3.2) is equivalent to

$$
\dot{\mathbf{x}}=\mathbf{v} \text {. }
$$

For comparison we state the matrix-vector formulation of Dziuk's semidiscretization (3.2)-(3.3), which is (3.5) together with

$$
\mathbf{M}^{[3]}(\mathbf{x}) \mathbf{v}+\mathbf{A}^{[3]}(\mathbf{x}) \mathbf{x}=\mathbf{0} .
$$

This is certainly more elegant than (3.4) and computationally less expensive. However, since the main computational cost is in the computation of the matrices $\mathbf{A}(\mathbf{x})$ and $\mathbf{M}(\mathbf{x})$ and their decompositions, the (fully discretized) scheme (3.4) is not substantially more expensive than the (fully discretized) scheme 
(3.6). On the other hand, the method presented here provides important extra geometrical information (about the normal vector and curvature) - and it comes with a proof of optimal-order convergence. The critical part in the convergence analysis is the proof of stability of the discretization, in the sense of bounding errors in terms of defects in the numerical equations in the appropriate norms. Our proof of stability uses the matrix-vector formulation (3.4) with (3.5), but it does not work for Dziuk's scheme (3.6) with (3.5).

\subsection{Lifts}

We need to compare functions on the exact surface $\Gamma(t)=\Gamma[X(\cdot, t)]$ with functions on the discrete surface $\Gamma_{h}(t)=\Gamma_{h}[\mathbf{x}(t)]$. To this end, we further work with functions on the interpolated surface $\Gamma_{h}^{*}(t)=\Gamma_{h}\left[\mathbf{x}^{*}(t)\right]$, where $\mathbf{x}^{*}(t)$ denotes the nodal vector collecting the grid points $x_{j}^{*}(t)=X\left(p_{j}, t\right)$ on the exact surface.

Any finite element function $w_{h}: \Gamma_{h}(t) \rightarrow \mathbb{R}^{m}(m=1$ or 3$)$ on the discrete surface, with nodal values $w_{j}$, is associated with a finite element function $\widehat{w}_{h}$ on the interpolated surface $\Gamma_{h}^{*}(t)$ that is defined by

$$
\widehat{w}_{h}=\sum_{j=1}^{N} w_{j} \phi_{j}\left[\mathbf{x}^{*}(t)\right] .
$$

This can be further lifted to a function on the exact surface by using the lift operator $l$, which was introduced for linear and higher-order surface approximations in [18] and [17], respectively. The lift operator $l$ maps a function on the interpolated surface $\Gamma_{h}^{*}$ to a function on the exact surface $\Gamma$, provided that $\Gamma_{h}^{*}$ is sufficiently close to $\Gamma$. The exact regular surface $\Gamma$ can be represented, in some neighbourhood of the surface, by a smooth signed distance function $d: \mathbb{R}^{3} \times[0, T] \rightarrow \mathbb{R}$, cf. [21, Section 2.1], such that $\Gamma$ is the zero level set of $d$ (i.e., $x \in \Gamma$ if and only if $d(x)=0$ ), with negative values of $d$ in the interior. Using this distance function, the lift of a continuous function $\eta_{h}: \Gamma_{h}^{*} \rightarrow \mathbb{R}^{m}$ is defined as $\eta_{h}^{l}(y):=\eta_{h}(x)$ for $x \in \Gamma_{h}^{*}$, where for every $x \in \Gamma_{h}^{*}$ the point $y=y(x) \in \Gamma$ is uniquely defined via $y=x-\nu(y) d(x)$.

The composed lift $L$ from finite element functions on the discrete surface $\Gamma_{h}(t)$ to functions on the exact surface $\Gamma(t)$ via the interpolated surface $\Gamma_{h}^{*}(t)$ is denoted by

$$
w_{h}^{L}=\left(\widehat{w}_{h}\right)^{l}
$$

\section{Convergence of the semi-discretization}

We are now in the position to formulate the first main result of this paper, which yields optimal-order error bounds for the finite element semidiscretization (3.1)-(3.2) of the system of mean curvature equations (2.6) with (2.1), for finite elements of polynomial degree $k \geq 2$. We denote by 
$\Gamma(t)=\Gamma[X(\cdot, t)]$ the exact surface and by $\Gamma_{h}(t)=\Gamma\left[X_{h}(\cdot, t)\right]=\Gamma_{h}[\mathbf{x}(t)]$ the discrete surface at time $t$. We introduce the notation

$$
x_{h}^{L}(x, t)=X_{h}^{L}(p, t) \in \Gamma_{h}(t) \quad \text { for } \quad x=X(p, t) \in \Gamma(t) .
$$

Theorem 4.1 Consider the space discretization (3.1)-(3.2) of the coupled mean curvature flow problem (2.6) with (2.1), using evolving surface finite elements of polynomial degree $k \geq 2$. Suppose that the mean curvature flow problem admits an exact solution $(X, v, \nu, H)$ that is sufficiently smooth on the time interval $t \in[0, T]$, and that the flow map $X(\cdot, t): \Gamma^{0} \rightarrow \Gamma(t) \subset \mathbb{R}^{3}$ is non-degenerate so that $\Gamma(t)$ is a regular surface on the time interval $t \in[0, T]$.

Then, there exists a constant $h_{0}>0$ such that for all mesh sizes $h \leq h_{0}$ the following error bounds for the lifts of the discrete position, velocity, normal vector and mean curvature hold over the exact surface $\Gamma(t)=\Gamma[X(\cdot, t)]$ for $0 \leq t \leq T$ :

$$
\begin{aligned}
\left\|x_{h}^{L}(\cdot, t)-\mathrm{id}_{\Gamma(t)}\right\|_{H^{1}(\Gamma(t))^{3}} & \leq C h^{k}, \\
\left\|v_{h}^{L}(\cdot, t)-v(\cdot, t)\right\|_{H^{1}(\Gamma(t))^{3}} & \leq C h^{k}, \\
\left\|\nu_{h}^{L}(\cdot, t)-\nu(\cdot, t)\right\|_{H^{1}(\Gamma(t))^{3}} & \leq C h^{k}, \\
\left\|H_{h}^{L}(\cdot, t)-H(\cdot, t)\right\|_{H^{1}(\Gamma(t))} & \leq C h^{k},
\end{aligned}
$$

and also

$$
\left\|X_{h}^{l}(\cdot, t)-X(\cdot, t)\right\|_{H^{1}\left(\Gamma_{0}\right)^{3}} \leq C h^{k},
$$

where the constant $C$ is independent of $h$ and $t$, but depends on bounds of the $H^{k+1}$ norms of the solution $(X, v, \nu, H)$ of the mean curvature flow and on the length $T$ of the time interval.

Sufficient regularity assumptions are the following: with bounds that are uniform in $t \in[0, T]$, we assume $X(\cdot, t) \in H^{k+1}\left(\Gamma^{0}\right), v(\cdot, t) \in H^{k+1}(\Gamma(X(\cdot, t)))$, and for $u=(\nu, H)$ we have $u(\cdot, t), \partial \bullet u(\cdot, t) \in W^{k+1, \infty}(\Gamma(X(\cdot, t)))^{4}$.

The proof of Theorem 4.1 is presented in Sections 7 to 9 , which are concerned with the stability and consistency of the numerical scheme, and the combination of the estimates. The proof of stability uses techniques that were first developed in our paper [33] on the finite element discretization of an evolving surface driven by the solution of a parabolic equation on the surface.

Several of the observations regarding the convergence result Theorem 3.1 of [33] apply also to Theorem 4.1, and so we restate them here:

A key issue in the proof is to ensure that the $W^{1, \infty}$ norm of the position error of the surfaces remains small. The $H^{1}$ error bound and an inverse estimate yield an $O\left(h^{k-1}\right)$ error bound in the $W^{1, \infty}$ norm. This is small only for $k \geq 2$, which is why we impose the condition $k \geq 2$ in the above result.

Since the exact flow map $X(\cdot, t): \Gamma^{0} \rightarrow \Gamma(t)$ is assumed to be smooth and non-degenerate, it is locally close to an invertible linear transformation, and (using compactness) it therefore preserves the admissibility of grids with sufficiently small mesh width $h \leq h_{0}$. Our assumptions therefore guarantee 
that the triangulations formed by the nodes $x_{j}^{*}(t)=X\left(p_{j}, t\right)$ remain admissible uniformly for $t \in[0, T]$ for sufficiently small $h$ (though the bounds in the admissibility inequalities and the largest possible mesh width may deteriorate with growing time). Since deformation is the gradient of position, the boundedness in $W^{1, \infty}$ of the position error (or equivalently, boundedness of deformation of the numerical flow map), which is ensured since the $O\left(h^{k}\right)$ error bound in $H^{1}$ and an inverse inequality yield an $O\left(h^{k-1}\right)$ error bound in $W^{1, \infty}$, excludes degeneration of the mesh on the numerical surface under the assumptions of the theorem.

The error bound will be proved by clearly separating the issues of consistency and stability. The consistency error is the defect that results from inserting a projection of the exact solution (here we use finite element interpolation) into the discretized equation. The defect bounds involve geometric estimates that were obtained for the time dependent case and for higher order $k \geq 2$ in [32, Section 5], by combining techniques of Dziuk \& Elliott [21,22] and Demlow [17]. This is done within the ESFEM formulation of Section 3.2.

The main difficulty in the proof of Theorem 4.1 is to prove stability in the form of an $h$-independent bound of the error in terms of the defect in appropriate norms. The stability analysis is done in the matrix-vector formulation of Section 3.3. It uses energy estimates and transport formulae that relate the mass and stiffness matrices and the coupling terms for different nodal vectors $\mathbf{x}$. No geometric estimates enter into the proof of stability.

While the basic approach to proving stability is the same as taken in [33], a different kind of energy estimates needs to be used, which allows us to derive $H^{1}$ error bounds uniformly on the time interval (and from this, uniform $W^{1, \infty}$ bounds via the inverse inequality), and not just $L^{2}\left(H^{1}\right)$ estimates as derived in [33]. These sharper estimates are needed because the nonlinearities in our evolution system (2.6) are only locally Lipschitz continuous. (In [33], global Lipschitz continuity of the nonlinearity $f(u, \nabla u)$ with respect to the second argument is assumed. This condition is used explicitly in the proof, although it was not clearly stated as an assumption in [33].)

As is explained in Remark 7.1 below, our techniques do not yield a proof of stability and convergence of Dziuk's method.

\section{Linearly implicit full discretization}

For the time discretization of the system of ordinary differential equations (3.4)-(3.5) we use a $q$-step linearly implicit backward difference formula (BDF) with $q \leq 5$. For a step size $\tau>0$, and with $t_{n}=n \tau \leq T$, we determine the approximations $\mathbf{x}^{n}$ to $\mathbf{x}\left(t_{n}\right), \mathbf{v}^{n}$ to $\mathbf{v}\left(t_{n}\right)$, and $\mathbf{u}^{n}$ to $\mathbf{u}\left(t_{n}\right)$ by the fully discrete 
system of linear equations

$$
\begin{aligned}
\mathbf{K}\left(\widetilde{\mathbf{x}}^{n}\right) \mathbf{v}^{n} & =\mathbf{g}\left(\widetilde{\mathbf{x}}^{n}, \widetilde{\mathbf{u}}^{n}\right), \\
\mathbf{M}\left(\widetilde{\mathbf{x}}^{n}\right) \frac{1}{\tau} \sum_{j=0}^{q} \delta_{j} \mathbf{u}^{n-j}+\mathbf{A}\left(\widetilde{\mathbf{x}}^{n}\right) \mathbf{u}^{n} & =\mathbf{f}\left(\widetilde{\mathbf{x}}^{n}, \widetilde{\mathbf{u}}^{n}\right), \\
\frac{1}{\tau} \sum_{j=0}^{q} \delta_{j} \mathbf{x}^{n-j} & =\mathbf{v}^{n},
\end{aligned}
$$

where $\widetilde{\mathbf{x}}^{n}$ and $\widetilde{\mathbf{u}}^{n}$ are the extrapolated values

$$
\widetilde{\mathbf{x}}^{n}=\sum_{j=0}^{q-1} \gamma_{j} \mathbf{x}^{n-1-j}, \quad \widetilde{\mathbf{u}}^{n}=\sum_{j=0}^{q-1} \gamma_{j} \mathbf{u}^{n-1-j}, \quad n \geq q
$$

The starting values $\mathbf{x}^{i}$ and $\mathbf{u}^{i}(i=0, \ldots, q-1)$ are assumed to be given. They can be precomputed using either a lower order method with smaller step sizes or an implicit Runge-Kutta method.

The method is determined by its coefficients, given by $\delta(\zeta)=\sum_{j=0}^{q} \delta_{j} \zeta^{j}=$ $\sum_{\ell=1}^{q} \frac{1}{\ell}(1-\zeta)^{\ell}$ and $\gamma(\zeta)=\sum_{j=0}^{q-1} \gamma_{j} \zeta^{j}=\left(1-(1-\zeta)^{q}\right) / \zeta$. The classical BDF method is known to be zero-stable for $q \leq 6$ and to have order $q$; see [29, Chapter V]. This order is retained by the linearly implicit variant using the above coefficients $\gamma_{j}$; cf. $[2,1]$.

We note that in the $n$th time step, the method requires solving two linear systems with the symmetric positive definite matrices $\mathbf{K}\left(\widetilde{\mathbf{x}}^{n}\right)$ and $\frac{\delta_{0}}{\tau} \mathbf{M}\left(\widetilde{\mathbf{x}}^{n}\right)+$ $\mathbf{A}\left(\widetilde{\mathbf{x}}^{n}\right)$.

From the vectors $\mathbf{x}^{n}=\left(x_{j}^{n}\right)$ and $\mathbf{v}^{n}=\left(v_{j}^{n}\right)$ we obtain position and velocity approximations to $X\left(\cdot, t_{n}\right), \operatorname{id}_{\Gamma\left[X\left(\cdot, t_{n}\right)\right]}$, and $v\left(\cdot, t_{n}\right)$ as

$$
\begin{array}{rlrl}
X_{h}^{n}\left(p_{h}\right) & =\sum_{j=1}^{N} x_{j}^{n} \phi_{j}[\mathbf{x}(0)]\left(p_{h}\right) \quad \text { for } p_{h} \in \Gamma_{h}^{0}, \\
x_{h}^{n} & =\operatorname{id}_{\Gamma\left[X_{h}^{n}\right]}, & \\
v_{h}^{n}(x) & =\sum_{j=1}^{N} v_{j}^{n} \phi_{j}\left[\mathbf{x}^{n}\right](x) & \text { for } x \in \Gamma_{h}\left[\mathbf{x}^{n}\right],
\end{array}
$$

and from the vectors $\mathbf{u}^{n}=\left(u_{j}^{n}\right)$ with $u_{j}^{n}=\left(\nu_{j}^{n}, H_{j}^{n}\right) \in \mathbb{R}^{3} \times \mathbb{R}$ we obtain approximations to the normal vector and the mean curvature at time $t_{n}$ as

$$
\begin{aligned}
& \nu_{h}^{n}(x)=\sum_{j=1}^{N} \nu_{j}^{n} \phi_{j}\left[\mathbf{x}^{n}\right](x) \quad \text { for } x \in \Gamma_{h}\left[\mathbf{x}^{n}\right], \\
& H_{h}^{n}(x)=\sum_{j=1}^{N} H_{j}^{n} \phi_{j}\left[\mathbf{x}^{n}\right](x) \quad \text { for } x \in \Gamma_{h}\left[\mathbf{x}^{n}\right] .
\end{aligned}
$$




\section{Convergence of the full discretization}

We are now in the position to formulate the second main result of this paper, which yields optimal-order error bounds for the combined ESFEM-BDF full discretization (5.1)-(5.4) of the system of mean curvature equations (2.6) with (2.1), for finite elements of polynomial degree $k \geq 2$ and BDF methods of order $q \leq 5$.

Theorem 6.1 Consider the ESFEM-BDF full discretization (5.1)-(5.4) of the coupled mean curvature flow problem (2.6) with (2.1), using evolving surface finite elements of polynomial degree $k \geq 2$ and linearly implicit BDF time discretization of order $q$ with $2 \leq q \leq 5$. Suppose that the mean curvature flow problem admits an exact solution $(X, v, \nu, H)$ that is sufficiently smooth on the time interval $t \in[0, T]$, and that the flow map $X(\cdot, t): \Gamma^{0} \rightarrow \Gamma(t) \subset \mathbb{R}^{3}$ is non-degenerate so that $\Gamma(t)$ is a regular surface on the time interval $t \in[0, T]$.

Then, there exist $h_{0}>0, \tau_{0}>0$, and $c_{0}>0$ such that for all mesh sizes $h \leq h_{0}$ and time step sizes $\tau \leq \tau_{0}$ satisfying the step size restriction

$$
\tau \leq C_{0} h
$$

(where $C_{0}>0$ can be chosen arbitrarily), the following error bounds for the lifts of the discrete position, velocity, normal vector and mean curvature hold over the exact surface: provided that the starting values are $O\left(h^{k}+\tau^{q+1 / 2}\right)$ accurate in the $H^{1}$ norm at time $t_{i}=i \tau$ for $i=0, \ldots, q-1$, we have at time $t_{n}=n \tau \leq T$

$$
\begin{aligned}
\left\|\left(x_{h}^{n}\right)^{L}-\operatorname{id}_{\Gamma\left(t_{n}\right)}\right\|_{H^{1}\left(\Gamma\left(t_{n}\right)\right)^{3}} & \leq C\left(h^{k}+\tau^{q}\right), \\
\left\|\left(v_{h}^{n}\right)^{L}-v\left(\cdot, t_{n}\right)\right\|_{H^{1}\left(\Gamma\left(t_{n}\right)\right)^{3}} & \leq C\left(h^{k}+\tau^{q}\right), \\
\left\|\left(\nu_{h}^{n}\right)^{L}-\nu\left(\cdot, t_{n}\right)\right\|_{H^{1}\left(\Gamma\left(t_{n}\right)\right)^{3}} & \leq C\left(h^{k}+\tau^{q}\right), \\
\left\|\left(H_{h}^{n}\right)^{L}-H\left(\cdot, t_{n}\right)\right\|_{H^{1}\left(\Gamma\left(t_{n}\right)\right)} & \leq C\left(h^{k}+\tau^{q}\right),
\end{aligned}
$$

and also

$$
\left\|\left(X_{h}^{n}\right)^{l}-X\left(\cdot, t_{n}\right)\right\|_{H^{1}\left(\Gamma_{0}\right)^{3}} \leq C\left(h^{k}+\tau^{q}\right),
$$

where the constant $C$ is independent of $h, \tau$ and $n$ with $n \tau \leq T$, but depends on bounds of higher derivatives of the solution $(X, v, \nu, H)$ of the mean curvature flow and on the length $T$ of the time interval, and on $C_{0}$.

Sufficient regularity assumptions are the following: uniformly in $t \in[0, T]$ and for $j=1, \ldots, q+1$,

$$
\begin{aligned}
& X(\cdot, t) \in H^{k+1}\left(\Gamma^{0}\right), \partial_{t}^{j} X(\cdot, t) \in H^{1}\left(\Gamma^{0}\right), \\
& v(\cdot, t) \in H^{k+1}(\Gamma(X(\cdot, t))), \partial^{\bullet j} v(\cdot, t) \in H^{2}(\Gamma(X(\cdot, t))), \\
\text { for } u=(\nu, H), \quad u(\cdot, t), \partial^{\bullet} u(\cdot, t) \in W^{k+1, \infty}(\Gamma(X(\cdot, t)))^{4} & \\
& \partial^{\bullet j} u(\cdot, t) \in H^{2}(\Gamma(X(\cdot, t)))^{4} .
\end{aligned}
$$


The proof of Theorem 6.1 is given in Sections 10 to 12. It uses results by Dahlquist [11] and Nevanlinna \& Odeh [38] in combination with the transfer of techniques of the proof of Theorem 4.1 to the time-discrete situation.

\section{Stability of the semi-discretization}

7.1 Preparation: Estimates relating different finite element surfaces

In our previous work [33, Section 4] we proved some technical results relating different finite element surfaces, which we recapitulate here. We use the following setting.

The finite element matrices of Section 3.3 induce discrete versions of Sobolev norms. Let $\mathbf{x} \in \mathbb{R}^{3 N}$ be a nodal vector defining the discrete surface $\Gamma_{h}[\mathbf{x}]$. For any nodal vector $\mathbf{w}=\left(w_{j}\right) \in \mathbb{R}^{N}$, with the corresponding finite element function $w_{h}=\sum_{j=1}^{N} w_{j} \phi_{j}[\mathbf{x}] \in S_{h}[\mathbf{x}]$, we define the following norms:

$$
\begin{aligned}
& \|\mathbf{w}\|_{\mathbf{M}(\mathbf{x})}^{2}=\mathbf{w}^{T} \mathbf{M}(\mathbf{x}) \mathbf{w}=\left\|w_{h}\right\|_{L^{2}\left(\Gamma_{h}[\mathbf{x}]\right)}^{2} \\
& \|\mathbf{w}\|_{\mathbf{A}(\mathbf{x})}^{2}=\mathbf{w}^{T} \mathbf{A}(\mathbf{x}) \mathbf{w}=\left\|\nabla_{\Gamma_{h}[\mathbf{x}]} w_{h}\right\|_{L^{2}\left(\Gamma_{h}[\mathbf{x}]\right)}^{2}, \\
& \|\mathbf{w}\|_{\mathbf{K}(\mathbf{x})}^{2}=\mathbf{w}^{T} \mathbf{K}(\mathbf{x}) \mathbf{w}=\left\|w_{h}\right\|_{H^{1}\left(\Gamma_{h}[\mathbf{x}]\right)}^{2}
\end{aligned}
$$

When $\mathbf{w} \in \mathbb{R}^{d N}$ so that the corresponding finite element function $w_{h}$ maps into $\mathbb{R}^{d}$, we write in the following $\left\|w_{h}\right\|_{L^{2}(\Gamma)}$ for $\left\|w_{h}\right\|_{L^{2}(\Gamma)^{d}}$ and $\left\|w_{h}\right\|_{H^{1}(\Gamma)}$ for $\left\|w_{h}\right\|_{H^{1}(\Gamma)^{d}}$.

Let now $\mathbf{x}, \mathbf{y} \in \mathbb{R}^{3 N}$ be two nodal vectors defining discrete surfaces $\Gamma_{h}[\mathbf{x}]$ and $\Gamma_{h}[\mathbf{y}]$, respectively. We denote the difference by $\mathbf{e}=\left(e_{j}\right)=\mathbf{x}-\mathbf{y} \in \mathbb{R}^{3 N}$. For $\theta \in[0,1]$, we consider the intermediate surface $\Gamma_{h}^{\theta}=\Gamma_{h}[\mathbf{y}+\theta \mathbf{e}]$ and the corresponding finite element functions given as

$$
e_{h}^{\theta}=\sum_{j=1}^{N} e_{j} \phi_{j}[\mathbf{y}+\theta \mathbf{e}]
$$

and in the same way, for any vectors $\mathbf{w}, \mathbf{z} \in \mathbb{R}^{N}$,

$$
w_{h}^{\theta}=\sum_{j=1}^{N} w_{j} \phi_{j}[\mathbf{y}+\theta \mathbf{e}] \quad \text { and } \quad z_{h}^{\theta}=\sum_{j=1}^{N} z_{j} \phi_{j}[\mathbf{y}+\theta \mathbf{e}]
$$

Figure 7.1 illustrates the described construction.

The following formulae relate the mass and stiffness matrices for the discrete surfaces $\Gamma_{h}[\mathbf{x}]$ and $\Gamma_{h}[\mathbf{y}]$. They result from the Leibniz rule and are given in Lemma 4.1 of [33]. 


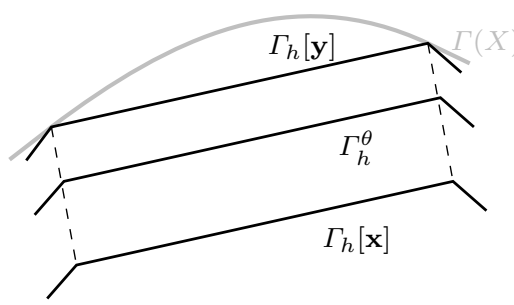

Fig. 7.1 The construction of the intermediate surfaces $\Gamma_{h}^{\theta}$

Lemma 7.1 In the above setting, the following identities hold true:

$$
\begin{aligned}
\mathbf{w}^{T}(\mathbf{M}(\mathbf{x})-\mathbf{M}(\mathbf{y})) \mathbf{z} & =\int_{0}^{1} \int_{\Gamma_{h}^{\theta}} w_{h}^{\theta}\left(\nabla_{\Gamma_{h}^{\theta}} \cdot e_{h}^{\theta}\right) z_{h}^{\theta} d \theta \\
\mathbf{w}^{T}(\mathbf{A}(\mathbf{x})-\mathbf{A}(\mathbf{y})) \mathbf{z} & =\int_{0}^{1} \int_{\Gamma_{h}^{\theta}} \nabla_{\Gamma_{h}^{\theta}} w_{h}^{\theta} \cdot\left(D_{\Gamma_{h}^{\theta}} e_{h}^{\theta}\right) \nabla_{\Gamma_{h}^{\theta}} z_{h}^{\theta} d \theta
\end{aligned}
$$

where $D_{\Gamma_{h}^{\theta}} e_{h}^{\theta}=\operatorname{tr}\left(E^{\theta}\right) I_{3}-\left(E^{\theta}+\left(E^{\theta}\right)^{T}\right)$ with $E^{\theta}=\nabla_{\Gamma_{h}^{\theta}} e_{h}^{\theta} \in \mathbb{R}^{3 \times 3}$.

The following lemma combines Lemmas 4.2 and 4.3 of [33].

Lemma 7.2 In the above setting, if

$$
\left\|\nabla_{\Gamma_{h}[\mathbf{y}]} e_{h}^{0}\right\|_{L^{\infty}\left(\Gamma_{h}[\mathbf{y}]\right)} \leq \frac{1}{2},
$$

then, for $0 \leq \theta \leq 1$ and $1 \leq p \leq \infty$, the finite element function $w_{h}^{\theta}=\sum_{j=1}^{N} w_{j} \phi_{j}[\mathbf{y}+\theta \mathbf{e}]$ on $\Gamma_{h}^{\theta}=\Gamma_{h}[\mathbf{y}+\theta \mathbf{e}]$ is bounded by

$$
\begin{aligned}
& \left\|w_{h}^{\theta}\right\|_{L^{p}\left(\Gamma_{h}^{\theta}\right)} \leq c_{p}\left\|w_{h}^{0}\right\|_{L^{p}\left(\Gamma_{h}^{0}\right)} \\
& \left\|\nabla_{\Gamma_{h}^{\theta}} w_{h}^{\theta}\right\|_{L^{p}\left(\Gamma_{h}^{\theta}\right)} \leq c_{p}\left\|\nabla_{\Gamma_{h}^{0}} w_{h}^{0}\right\|_{L^{p}\left(\Gamma_{h}^{0}\right)}
\end{aligned}
$$

where $c_{p}$ is an absolute constant (in particular, independent of $0 \leq \theta \leq 1$ and $h$ ). Moreover, $c_{\infty}=2$.

The first estimate is not stated explicitly in [33], but follows immediately with the proof of Lemma 4.3 in [33].

If $\left\|\nabla_{\Gamma_{h}[\mathbf{y}]} e_{h}^{0}\right\|_{L^{\infty}\left(\Gamma_{h}[\mathbf{y}]\right)} \leq \frac{1}{4}$, using the lemma for $w_{h}^{\theta}=e_{h}^{\theta}$ shows that

$$
\left\|\nabla_{\Gamma_{h}^{\theta}} e_{h}^{\theta}\right\|_{L^{\infty}\left(\Gamma_{h}^{\theta}\right)} \leq \frac{1}{2}, \quad 0 \leq \theta \leq 1,
$$

and then the lemma with $p=2$ and the definition of the norms (7.1) and (7.2) (and interchanging the roles of $\mathbf{y}$ and $\mathbf{y}+\theta \mathbf{e}$ ) show that

the norms $\|\cdot\|_{\mathbf{M}(\mathbf{y}+\theta \mathbf{e})}$ are $h$-uniformly equivalent for $0 \leq \theta \leq 1$, and so are the norms $\|\cdot\|_{\mathbf{A}(\mathbf{y}+\theta \mathbf{e})}$. 
Under the condition that $\varepsilon:=\left\|\nabla_{\Gamma_{h}[\mathbf{y}]} e_{h}^{0}\right\|_{L^{\infty}\left(\Gamma_{h}[\mathbf{y}]\right)} \leq \frac{1}{4}$, using (7.6) in Lemma 7.1 and applying the Cauchy-Schwarz inequality yields the bounds, with $c=c_{\infty} c_{2}^{2}$,

$$
\begin{gathered}
\mathbf{w}^{T}(\mathbf{M}(\mathbf{x})-\mathbf{M}(\mathbf{y})) \mathbf{z} \leq c \varepsilon\|\mathbf{w}\|_{\mathbf{M}(\mathbf{y})}\|\mathbf{z}\|_{\mathbf{M}(\mathbf{y})}, \\
\mathbf{w}^{T}(\mathbf{A}(\mathbf{x})-\mathbf{A}(\mathbf{y})) \mathbf{z} \leq c \varepsilon\|\mathbf{w}\|_{\mathbf{A}(\mathbf{y})}\|\mathbf{z}\|_{\mathbf{A}(\mathbf{y})} .
\end{gathered}
$$

We will also use similar bounds where we use the $L^{\infty}$ norm of $w_{h}$ or its gradient and the $L^{2}$ norm of the gradient of $e_{h}$.

Consider now a continuously differentiable function $\mathbf{x}:[0, T] \rightarrow \mathbb{R}^{3 N}$ that defines a finite element surface $\Gamma_{h}[\mathbf{x}(t)]$ for every $t \in[0, T]$, and assume that its time derivative $\mathbf{v}(t)=\dot{\mathbf{x}}(t)$ is the nodal vector of a finite element function $v_{h}(\cdot, t)$ that satisfies

$$
\left\|\nabla_{\Gamma_{h}[\mathbf{x}(t)]} v_{h}(\cdot, t)\right\|_{L^{\infty}\left(\Gamma_{h}[\mathbf{x}(t)]\right)} \leq K, \quad 0 \leq t \leq T .
$$

With $\mathbf{e}=\mathbf{x}(t)-\mathbf{x}(s)=\int_{s}^{t} \mathbf{v}(r) d r$, the bounds (7.8) then yield the following bounds, which were first shown in Lemma 4.1 of [24]:

for $0 \leq s, t \leq T$ with $K|t-s| \leq \frac{1}{4}$, we have with $C=c K$

$$
\begin{gathered}
\mathbf{w}^{T}(\mathbf{M}(\mathbf{x}(t))-\mathbf{M}(\mathbf{x}(s))) \mathbf{z} \leq C|t-s|\|\mathbf{w}\|_{\mathbf{M}(\mathbf{x}(t))}\|\mathbf{z}\|_{\mathbf{M}(\mathbf{x}(t))} \\
\mathbf{w}^{T}(\mathbf{A}(\mathbf{x}(t))-\mathbf{A}(\mathbf{x}(s))) \mathbf{z} \leq C|t-s|\|\mathbf{w}\|_{\mathbf{A}(\mathbf{x}(t))}\|\mathbf{z}\|_{\mathbf{A}(\mathbf{x}(t))}
\end{gathered}
$$

Letting $s \rightarrow t$, this implies the bounds stated in Lemma 4.6 of [33]:

$$
\begin{gathered}
\mathbf{w}^{T} \frac{\mathrm{d}}{\mathrm{d} t} \mathbf{M}(\mathbf{x}(t)) \mathbf{z} \leq C\|\mathbf{w}\|_{\mathbf{M}(\mathbf{x}(t))}\|\mathbf{z}\|_{\mathbf{M}(\mathbf{x}(t))}, \\
\mathbf{w}^{T} \frac{\mathrm{d}}{\mathrm{d} t} \mathbf{A}(\mathbf{x}(t)) \mathbf{z} \leq C\|\mathbf{w}\|_{\mathbf{A}(\mathbf{x}(t))}\|\mathbf{z}\|_{\mathbf{A}(\mathbf{x}(t))} .
\end{gathered}
$$

Moreover, by patching together finitely many intervals over which $K|t-s| \leq \frac{1}{4}$, we obtain that

the norms $\|\cdot\|_{\mathbf{M}(\mathbf{x}(t))}$ are $h$-uniformly equivalent for $0 \leq t \leq T$, and so are the norms $\|\cdot\|_{\mathbf{A}(\mathbf{x}(t))}$.

\subsection{Defects and errors}

We choose nodal vectors $\mathbf{x}^{*}(t) \in \mathbb{R}^{3 N}, \mathbf{v}^{*}(t) \in \mathbb{R}^{3 N}$ and $\mathbf{u}^{*}(t) \in \mathbb{R}^{4 N}$ that are related to the exact solution $X, v$ and $u=(\nu, H)$ as follows: $\mathbf{x}^{*}(t)$ and $\mathbf{v}^{*}(t)$ collect the values at the finite element nodes of $X(\cdot, t)$ and $v(\cdot, t)$, respectively. The vector $\mathbf{u}^{*}(t)$ contains the nodal values of the finite element function 
$u_{h}^{*}(\cdot, t) \in S_{h}\left[\mathbf{x}^{*}(t)\right]^{4}$ that is defined on the interpolated surface $\Gamma_{h}\left[\mathbf{x}^{*}(t)\right]$ by a Ritz map: omitting the ubiquitous argument $t$,

$$
\int_{\Gamma_{h}\left[\mathbf{x}^{*}\right]} \nabla_{\Gamma_{h}\left[\mathbf{x}^{*}\right]} u_{h}^{*} \cdot \nabla_{\Gamma_{h}\left[\mathbf{x}^{*}\right]} \varphi_{h}+\int_{\Gamma_{h}\left[\mathbf{x}^{*}\right]} u_{h}^{*} \cdot \varphi_{h}=\int_{\Gamma[X]} \nabla_{\Gamma[X]} u \cdot \nabla_{\Gamma[X]} \varphi_{h}^{l}+\int_{\Gamma[X]} u \cdot \varphi_{h}^{l}
$$

for all $\varphi_{h} \in S_{h}\left[\mathbf{x}^{*}\right]^{4}$, where again $\varphi_{h}^{l}$ denotes the lift to a function on $\Gamma[X]$.

The nodal vectors $\mathbf{x}^{*}(t), \mathbf{v}^{*}(t), \mathbf{u}^{*}(t)$ satisfy the equations (3.4) up to some defects $\mathbf{d}_{\mathbf{v}}$ and $\mathbf{d}_{\mathbf{u}}$ that will be studied in Section 8:

$$
\begin{aligned}
\mathbf{K}^{[3]}\left(\mathbf{x}^{*}\right) \mathbf{v}^{*} & =\mathbf{g}\left(\mathbf{x}^{*}, \mathbf{u}^{*}\right)+\mathbf{M}^{[3]}\left(\mathbf{x}^{*}\right) \mathbf{d}_{\mathbf{v}}, \\
\mathbf{M}^{[4]}\left(\mathbf{x}^{*}\right) \dot{\mathbf{u}}^{*}+\mathbf{A}^{[4]}\left(\mathbf{x}^{*}\right) \mathbf{u}^{*} & =\mathbf{f}\left(\mathbf{x}^{*}, \mathbf{u}^{*}\right)+\mathbf{M}^{[4]}\left(\mathbf{x}^{*}\right) \mathbf{d}_{\mathbf{u}},
\end{aligned}
$$

together with the differential equation

$$
\dot{\mathbf{x}}^{*}=\mathbf{v}^{*} .
$$

In the following we omit the dimension superscripts [3] and [4] on the matrices $\mathbf{M}, \mathbf{A}$ and $\mathbf{K}=\mathbf{M}+\mathbf{A}$.

The errors between the nodal values of the numerical solutions and the exact values are denoted by $\mathbf{e}_{\mathbf{x}}=\mathbf{x}-\mathbf{x}^{*}, \mathbf{e}_{\mathbf{v}}=\mathbf{v}-\mathbf{v}^{*}, \mathbf{e}_{\mathbf{u}}=\mathbf{u}-\mathbf{u}^{*}$ and their corresponding finite element functions on the interpolated surface $\Gamma_{h}\left[\mathbf{x}^{*}\right]$ by $e_{x}, e_{v}, e_{u}$, respectively. We obtain the error equations by subtracting (7.14) from (3.4) and (7.15) from (3.5):

$$
\begin{aligned}
\mathbf{K}(\mathbf{x}) \mathbf{e}_{\mathbf{v}}= & -\left(\mathbf{K}(\mathbf{x})-\mathbf{K}\left(\mathbf{x}^{*}\right)\right) \mathbf{v}^{*} \\
& +\left(\mathbf{g}(\mathbf{x}, \mathbf{u})-\mathbf{g}\left(\mathbf{x}^{*}, \mathbf{u}^{*}\right)\right)-\mathbf{M}\left(\mathbf{x}^{*}\right) \mathbf{d}_{\mathbf{v}}, \\
\mathbf{M}(\mathbf{x}) \dot{\mathbf{e}}_{\mathbf{u}}+\mathbf{A}(\mathbf{x}) \mathbf{e}_{\mathbf{u}}= & -\left(\mathbf{M}(\mathbf{x})-\mathbf{M}\left(\mathbf{x}^{*}\right)\right) \dot{\mathbf{u}}^{*} \\
& -\left(\mathbf{A}(\mathbf{x})-\mathbf{A}\left(\mathbf{x}^{*}\right)\right) \mathbf{u}^{*} \\
& +\left(\mathbf{f}(\mathbf{x}, \mathbf{u})-\mathbf{f}\left(\mathbf{x}^{*}, \mathbf{u}^{*}\right)\right)-\mathbf{M}\left(\mathbf{x}^{*}\right) \mathbf{d}_{\mathbf{u}}, \\
\dot{\mathbf{e}}_{\mathbf{x}}= & \mathbf{e}_{\mathbf{v}} .
\end{aligned}
$$

We note further that $\mathbf{e}_{\mathbf{x}}(0)=0$ and $\mathbf{e}_{\mathbf{v}}(0)=0$, but in general $\mathbf{e}_{\mathbf{u}}(0) \neq 0$.

For estimating the defect $\mathbf{d}_{\mathbf{v}}$, we use the norm given by

$$
\left\|\mathbf{d}_{\mathbf{v}}\right\|_{*, \mathbf{x}^{*}}^{2}:=\mathbf{d}_{\mathbf{v}}^{T} \mathbf{M}\left(\mathbf{x}^{*}\right) \mathbf{K}\left(\mathbf{x}^{*}\right)^{-1} \mathbf{M}\left(\mathbf{x}^{*}\right) \mathbf{d}_{\mathbf{v}} .
$$

By [33, Eq. (5.5)], this equals the following dual norm for the corresponding finite element function $d_{v} \in S_{h}\left[\mathbf{x}^{*}\right]^{3}$, which has the vector of nodal values $\mathbf{d}_{\mathbf{v}}$,

$$
\left\|\mathbf{d}_{\mathbf{v}}\right\|_{\star, \mathbf{x}^{*}}=\left\|d_{v}\right\|_{H_{h}^{-1}\left(\Gamma_{h}\left[\mathbf{x}^{*}\right]\right)}:=\sup _{0 \neq \varphi_{h} \in S_{h}\left[\mathbf{x}^{*}\right]^{3}} \frac{\int_{\Gamma_{h}\left[\mathbf{x}^{*}\right]} d_{v} \cdot \varphi_{h}}{\left\|\varphi_{h}\right\|_{H^{1}\left(\Gamma_{h}\left[\mathbf{x}^{*}\right]\right)}} .
$$

For estimating the defect $\mathbf{d}_{\mathbf{u}}$, which is the nodal vector of a finite element function $d_{u} \in S_{h}\left(\mathbf{x}^{*}\right)^{4}$, we use the $L^{2}$ norm

$$
\left\|\mathbf{d}_{\mathbf{u}}\right\|_{\mathbf{M}\left(\mathbf{x}^{*}\right)}^{2}=\mathbf{d}_{\mathbf{u}}^{T} \mathbf{M}\left(\mathbf{x}^{*}\right) \mathbf{d}_{\mathbf{u}}=\left\|d_{u}\right\|_{L^{2}\left(\Gamma_{h}\left[\mathbf{x}^{*}\right]\right)}^{2} .
$$

The errors will be estimated in the $H^{1}$ norm:

$$
\|\mathbf{e}\|_{\mathbf{K}\left(\mathbf{x}^{*}\right)}^{2}=\mathbf{e}^{T} \mathbf{K}\left(\mathbf{x}^{*}\right) \mathbf{e}=\|e\|_{H^{1}\left(\Gamma_{h}\left[\mathbf{x}^{*}\right]\right)}^{2} .
$$


7.3 Stability estimate

The following result provides the key stability estimate.

Proposition 7.1 Assume that, for some $\kappa$ with $1<\kappa \leq k$, the defects are bounded by

$$
\begin{aligned}
\left\|\mathbf{d}_{\mathbf{v}}(t)\right\|_{\star, \mathbf{x}^{*}(t)} & \leq c h^{\kappa}, \quad \text { for } \quad 0 \leq t \leq T, \\
\left\|\mathbf{d}_{\mathbf{u}}(t)\right\|_{\mathbf{M}\left(\mathbf{x}^{*}(t)\right)} & \leq c h^{\kappa},
\end{aligned}
$$

and that also the errors in the initial values satisfy

$$
\left\|\mathbf{e}_{\mathbf{u}}(0)\right\|_{\mathbf{K}\left(\mathbf{x}^{*}(0)\right)} \leq c h^{\kappa}
$$

Then, there exists $h_{0}>0$ such that the following stability estimate holds for all $h \leq h_{0}$ and $0 \leq t \leq T$ :

$$
\begin{aligned}
& \left\|\mathbf{e}_{\mathbf{x}}(t)\right\|_{\mathbf{K}\left(\mathbf{x}^{*}(t)\right)}^{2}+\left\|\mathbf{e}_{\mathbf{v}}(t)\right\|_{\mathbf{K}\left(\mathbf{x}^{*}(t)\right)}^{2}+\left\|\mathbf{e}_{\mathbf{u}}(t)\right\|_{\mathbf{K}\left(\mathbf{x}^{*}(t)\right)}^{2} \\
& \leq C\left\|\mathbf{e}_{\mathbf{u}}(0)\right\|_{\mathbf{K}\left(\mathbf{x}^{*}(0)\right)}^{2}+C \max _{0 \leq s \leq t}\left\|\mathbf{d}_{\mathbf{v}}(s)\right\|_{\star, \mathbf{x}^{*}(s)}^{2}+C \int_{0}^{t}\left\|\mathbf{d}_{\mathbf{u}}(s)\right\|_{\mathbf{M}\left(\mathbf{x}^{*}(s)\right)}^{2} d s,
\end{aligned}
$$

where $C$ is independent of $h$ and $t$, but depends on the final time $T$.

From the interpolation error bound of Proposition 2.7 in [17] and the Ritz map error bound of Theorem 6.3 in [32] we know that $\left\|\mathbf{e}_{\mathbf{u}}(0)\right\|_{\mathbf{K}\left(\mathbf{x}^{*}(0)\right)}=O\left(h^{k}\right)$. We note that therefore the error functions $e_{x}(\cdot, t), e_{v}(\cdot, t), e_{u}(\cdot, t) \in S_{h}\left[\mathbf{x}^{*}(t)\right]$ corresponding to the nodal values $\mathbf{e}_{\mathbf{x}}, \mathbf{e}_{\mathbf{v}}, \mathbf{e}_{\mathbf{u}}$, respectively, are bounded by

$$
\begin{aligned}
\left\|e_{x}(\cdot, t)\right\|_{H^{1}\left(\Gamma_{h}\left[\mathbf{x}^{*}(t)\right]\right)} & +\left\|e_{v}(\cdot, t)\right\|_{H^{1}\left(\Gamma_{h}\left[\mathbf{x}^{*}(t)\right]\right)} \\
& +\left\|e_{u}(\cdot, t)\right\|_{H^{1}\left(\Gamma_{h}\left[\mathbf{x}^{*}(t)\right]\right)} \leq C h^{\kappa} .
\end{aligned}
$$

In Section 8 we will show that the assumed defect bounds (7.17) are indeed satisfied with $\kappa=k \geq 2$, provided the exact solution is sufficiently smooth.

Proof The basic procedure of the proof follows that of [33]: it uses energy estimates for the error equations in the matrix-vector formulation and relies on the technical lemmas of Section 7.1. Since we need uniform-in-time $H^{1}$ norm error bounds (in order to control the $W^{1, \infty}$ norm of the errors in $u$ via an inverse inequality), we test with the time derivative of the error vector. In contrast, in [33] we just tested with the error vector as this allowed us to obtain $L^{2}\left(H^{1}\right)$-norm estimates for $e_{u}$, which was sufficient for a nonlinearity $f\left(u, \nabla_{\Gamma} u\right)$ that is globally Lipschitz-bounded in the second variable. This global Lipschitz continuity is, however, not satisfied for the nonlinearity considered here.

We test (7.16a) with $\mathbf{e}_{\mathbf{v}}$, which yields

$$
\begin{aligned}
\left\|\mathbf{e}_{\mathbf{v}}\right\|_{\mathbf{K}(\mathbf{x})}^{2}=\mathbf{e}_{\mathbf{v}}^{T} \mathbf{K}(\mathbf{x}) \mathbf{e}_{\mathbf{v}}= & -\mathbf{e}_{\mathbf{v}}^{T}\left(\mathbf{K}(\mathbf{x})-\mathbf{K}\left(\mathbf{x}^{*}\right)\right) \mathbf{v}^{*} \\
& +\mathbf{e}_{\mathbf{v}}^{T}\left(\mathbf{g}(\mathbf{x}, \mathbf{u})-\mathbf{g}\left(\mathbf{x}^{*}, \mathbf{u}^{*}\right)\right)-\mathbf{e}_{\mathbf{v}}^{T} \mathbf{M}\left(\mathbf{x}^{*}\right) \mathbf{d}_{\mathbf{v}},
\end{aligned}
$$


while we test (7.16b) with $\dot{\mathbf{e}}_{\mathbf{u}}$, which yields

$$
\begin{aligned}
\dot{\mathbf{e}}_{\mathbf{u}}^{T} \mathbf{M}(\mathbf{x}) \dot{\mathbf{e}}_{\mathbf{u}}+\dot{\mathbf{e}}_{\mathbf{u}}^{T} \mathbf{A}(\mathbf{x}) \mathbf{e}_{\mathbf{u}}= & -\dot{\mathbf{e}}_{\mathbf{u}}^{T}\left(\mathbf{M}(\mathbf{x})-\mathbf{M}\left(\mathbf{x}^{*}\right)\right) \dot{\mathbf{u}}^{*} \\
& -\dot{\mathbf{e}}_{\mathbf{u}}^{T}\left(\mathbf{A}(\mathbf{x})-\mathbf{A}\left(\mathbf{x}^{*}\right)\right) \mathbf{u}^{*} \\
& +\dot{\mathbf{e}}_{\mathbf{u}}^{T}\left(\mathbf{f}(\mathbf{x}, \mathbf{u})-\mathbf{f}\left(\mathbf{x}^{*}, \mathbf{u}^{*}\right)\right) \\
& -\dot{\mathbf{e}}_{\mathbf{u}}^{T} \mathbf{M}\left(\mathbf{x}^{*}\right) \mathbf{d}_{\mathbf{u}} .
\end{aligned}
$$

Let $t^{*} \in(0, T]$ be the maximal time such that the following inequalities hold:

$$
\begin{aligned}
\left\|e_{x}(\cdot, t)\right\|_{W^{1, \infty}\left(\Gamma_{h}\left[\mathbf{x}^{*}(t)\right]\right)} & \leq h^{(\kappa-1) / 2}, \\
\left\|e_{v}(\cdot, t)\right\|_{W^{1, \infty}\left(\Gamma_{h}\left[\mathbf{x}^{*}(t)\right]\right)} \leq h^{(\kappa-1) / 2}, & \text { for } \quad t \in\left[0, t^{*}\right] . \\
\left\|e_{u}(\cdot, t)\right\|_{W^{1, \infty}\left(\Gamma_{h}\left[\mathbf{x}^{*}(t)\right]\right)} \leq h^{(\kappa-1) / 2}, &
\end{aligned}
$$

Note that $t^{*}>0$ since initially $e_{x}(\cdot, 0)=0, e_{v}(\cdot, 0)=0$ and, by an inverse inequality and the higher-order error bound for the Ritz map [32], we have $\left\|e_{u}(\cdot, 0)\right\|_{W^{1, \infty}\left(\Gamma_{h}\left[\mathbf{x}^{*}(0)\right]\right)} \leq c h^{-1}\left\|e_{u}(\cdot, 0)\right\|_{H^{1}\left(\Gamma_{h}\left[\mathbf{x}^{*}(0)\right]\right)} \leq C h^{k-1}$. We first prove the stated error bounds for $0 \leq t \leq t^{*}$. At the end of the proof we will show that in fact $t^{*}$ coincides with $T$.

Since the exact surface functions $X, v, u$ are assumed smooth, the associated finite element functions $x_{h}^{*}(\cdot, t), v_{h}^{*}(\cdot, t), u_{h}^{*}(\cdot, t)$ on the interpolated surface $\Gamma_{h}\left[\mathbf{x}^{*}(t)\right]$ have $W^{1, \infty}$ norms that are bounded independently of $h$, for all $t \in[0, T]$. The bounds (7.21) together with Lemma 7.2 then imply that the $W^{1, \infty}$ norms of the ESFEM functions $x_{h}(\cdot, t), v_{h}(\cdot, t), u_{h}(\cdot, t)$ on the discrete surface $\Gamma_{h}[\mathbf{x}(t)]$ are also bounded independently of $h$ and $t \in\left[0, t^{*}\right]$, and so are their lifts to the interpolated surface $\Gamma_{h}\left[\mathbf{x}^{*}(t)\right]$. In particular, it will be important that the discrete velocity $v_{h}$ with nodal vector $\mathbf{v}=\dot{\mathbf{x}}$ satisfies $(7.9)$.

In the following $c$ and $C$ are generic constants that take different values on different occurrences.

(A) Estimates for the surface PDE: We estimate the terms of (7.20) separately, with Lemmas 7.1 and 7.2 and the ensuing bounds (7.7)-(7.11) as our main tools. The condition of Lemma 7.2 follows from the first estimate in (7.21), $\left\|e_{x}(\cdot, t)\right\|_{W^{1, \infty}\left(\Gamma_{h}\left[\mathbf{x}^{*}(t)\right]\right)} \leq h^{(\kappa-1) / 2} \leq \frac{1}{2}$ for $h \leq h_{0}$ sufficiently small.

(i) By the definition of the $\mathbf{M}$-norm we have

$$
\dot{\mathbf{e}}_{\mathbf{u}}^{T} \mathbf{M}(\mathbf{x}) \dot{\mathbf{e}}_{\mathbf{u}}=\left\|\dot{\mathbf{e}}_{\mathbf{u}}\right\|_{\mathbf{M}(\mathbf{x})}^{2} .
$$

(ii) The symmetry of $\mathbf{A}(\mathbf{x})$ and the bound (7.11) yield

$$
\begin{aligned}
\dot{\mathbf{e}}_{\mathbf{u}}^{T} \mathbf{A}(\mathbf{x}) \mathbf{e}_{\mathbf{u}} & =-\frac{1}{2} \mathbf{e}_{\mathbf{u}}^{T} \frac{\mathrm{d}}{\mathrm{d} t}(\mathbf{A}(\mathbf{x})) \mathbf{e}_{\mathbf{u}}+\frac{1}{2} \frac{\mathrm{d}}{\mathrm{d} t}\left(\mathbf{e}_{\mathbf{u}}^{T} \mathbf{A}(\mathbf{x}) \mathbf{e}_{\mathbf{u}}\right) \\
& \geq-c\left\|\mathbf{e}_{\mathbf{u}}\right\|_{\mathbf{A}(\mathbf{x})}^{2}+\frac{1}{2} \frac{\mathrm{d}}{\mathrm{d} t}\left\|\mathbf{e}_{\mathbf{u}}\right\|_{\mathbf{A}(\mathbf{x})}^{2} .
\end{aligned}
$$

(iii) Using Lemmas 7.1 and 7.2, we now show for the first term on the right-hand side that

$$
-\dot{\mathbf{e}}_{\mathbf{u}}^{T}\left(\mathbf{M}(\mathbf{x})-\mathbf{M}\left(\mathbf{x}^{*}\right)\right) \dot{\mathbf{u}}^{*} \leq C\left\|\dot{\mathbf{e}}_{\mathbf{u}}\right\|_{\mathbf{M}\left(\mathbf{x}^{*}\right)}\left\|\mathbf{e}_{\mathbf{x}}\right\|_{\mathbf{A}\left(\mathbf{x}^{*}\right)}
$$


with a constant $C$ that is independent of $h$.

By Lemma 7.1 we have with $\Gamma_{h}^{\theta}=\Gamma_{h}\left[\mathbf{x}^{*}+\theta \mathbf{e}_{\mathbf{x}}\right]$

$$
\begin{aligned}
-\dot{\mathbf{e}}_{\mathbf{u}}^{T}\left(\mathbf{M}(\mathbf{x})-\mathbf{M}\left(\mathbf{x}^{*}\right)\right) \dot{\mathbf{u}}^{*} & =-\int_{0}^{1} \int_{\Gamma_{h}^{\theta}} \dot{e}_{u}^{\theta}\left(\nabla_{\Gamma_{h}^{\theta}} \cdot e_{x}^{\theta}\right) \partial_{h}^{\bullet} u_{h}^{*, \theta} \mathrm{d} \theta \\
& \leq \int_{0}^{1}\left\|\dot{e}_{u}^{\theta}\right\|_{L^{2}\left(\Gamma_{h}^{\theta}\right)}\left\|\nabla_{\Gamma_{h}^{\theta}} \cdot e_{x}^{\theta}\right\|_{L^{2}\left(\Gamma_{h}^{\theta}\right)}\left\|\partial_{h}^{\bullet} u_{h}^{*, \theta}\right\|_{L^{\infty}\left(\Gamma_{h}^{\theta}\right)} \mathrm{d} \theta .
\end{aligned}
$$

Here we note that by Lemma 7.2 together with the bound (7.21),

$$
\begin{aligned}
\left\|\nabla_{\Gamma_{h}^{\theta}} \cdot e_{x}^{\theta}\right\|_{L^{2}\left(\Gamma_{h}^{\theta}\right)} & \leq\left\|\nabla_{\Gamma_{h}^{\theta}} e_{x}^{\theta}\right\|_{L^{2}\left(\Gamma_{h}^{\theta}\right)} \\
& \leq c_{2}\left\|\nabla_{\Gamma_{h}^{0}} e_{x}^{0}\right\|_{L^{2}\left(\Gamma_{h}^{0}\right)}=c_{2}\left\|\nabla_{\Gamma_{h}\left[\mathbf{x}^{*}\right]} e_{x}\right\|_{L^{2}\left(\Gamma_{h}\left[\mathbf{x}^{*}\right]\right)}
\end{aligned}
$$

and also

$$
\left\|\dot{e}_{u}^{\theta}\right\|_{L^{2}\left(\Gamma_{h}^{\theta}\right)} \leq c_{2}\left\|\dot{e}_{u}^{0}\right\|_{L^{2}\left(\Gamma_{h}^{0}\right)}=c_{2}\left\|\dot{e}_{u}\right\|_{L^{2}\left(\Gamma_{h}\left[\mathbf{x}^{*}\right]\right)}
$$

and

$$
\left\|\partial_{h}^{\bullet} u_{h}^{*, \theta}\right\|_{L^{\infty}\left(\Gamma_{h}^{\theta}\right)} \leq c_{\infty}\left\|\partial_{h}^{\bullet} u_{h}^{*, 0}\right\|_{L^{\infty}\left(\Gamma_{h}^{0}\right)}=c_{\infty}\left\|\partial_{h}^{\bullet} u_{h}^{*}\right\|_{L^{\infty}\left(\Gamma_{h}\left[\mathbf{x}^{*}\right]\right)} .
$$

We now show that the last term is bounded by a constant that depends only on the solution regularity. For the material derivative of the Ritz map we have

$$
\begin{aligned}
& \left\|\partial_{h}^{\bullet} u_{h}^{*}\right\|_{L^{\infty}\left(\Gamma_{h}\left[\mathbf{x}^{*}\right]\right)} \leq c\left\|\left(\partial_{h}^{\bullet} u_{h}^{*}\right)^{l}\right\|_{L^{\infty}(\Gamma[X(\cdot, t)])} \\
& \leq c\left\|\left(\partial_{h}^{\bullet} u_{h}^{*}\right)^{l}-I_{h} \partial^{\bullet} u\right\|_{L^{\infty}(\Gamma[X(\cdot, t)])} \\
& \quad+c\left\|I_{h} \partial^{\bullet} u-\partial^{\bullet} u\right\|_{L^{\infty}(\Gamma[X(\cdot, t)])}+c\left\|\partial^{\bullet} u\right\|_{L^{\infty}(\Gamma[X(\cdot, t)])} \\
& \leq \frac{c}{h}\left\|\left(\partial_{h}^{\bullet} u_{h}^{*}\right)^{l}-I_{h} \partial^{\bullet} u\right\|_{L^{2}(\Gamma[X(\cdot, t)])} \\
& \quad+c\left\|I_{h} \partial^{\bullet} u-\partial^{\bullet} u\right\|_{L^{\infty}(\Gamma[X(\cdot, t)])}+c\left\|\partial^{\bullet} u\right\|_{L^{\infty}(\Gamma[X(\cdot, t)])} \\
& \leq \frac{c}{h}\left\|\left(\partial_{h}^{\bullet} u_{h}^{*}\right)^{l}-\partial^{\bullet} u\right\|_{L^{2}(\Gamma[X(\cdot, t)])}+\frac{c}{h}\left\|\partial^{\bullet} u-I_{h} \partial \bullet u\right\|_{L^{2}(\Gamma[X(\cdot, t)])} \\
& \quad+c\left\|I_{h} \partial^{\bullet} u-\partial^{\bullet} u\right\|_{L^{\infty}(\Gamma[X(\cdot, t)])}+c\left\|\partial^{\bullet} u\right\|_{L^{\infty}(\Gamma[X(\cdot, t)])} \\
& \leq C h^{k-1}+C h^{k}+C h^{k}+C,
\end{aligned}
$$

where we used the norm equivalence for the lift operator (see [17]) in the first inequality, an inverse inequality in the second inequality, and the known error bounds for interpolation (see [17, Proposition 2]) and for the Ritz map (see [32, Theorem 6.3]) in the last inequality. Combining the estimates above yields

$$
\begin{aligned}
-\dot{\mathbf{e}}_{\mathbf{u}}^{T}\left(\mathbf{M}(\mathbf{x})-\mathbf{M}\left(\mathbf{x}^{*}\right)\right) \dot{\mathbf{u}}^{*} & \leq C\left\|\dot{e}_{u}\right\|_{L^{2}\left(\Gamma_{h}\left[\mathbf{x}^{*}\right]\right)}\left\|\nabla_{\Gamma_{h}\left[\mathbf{x}^{*}\right]} e_{x}\right\|_{L^{2}\left(\Gamma_{h}\left[\mathbf{x}^{*}\right]\right)} \\
& =C\left\|\dot{\mathbf{e}}_{\mathbf{u}}\right\|_{\mathbf{M}\left(\mathbf{x}^{*}\right)}\left\|\mathbf{e}_{\mathbf{x}}\right\|_{\mathbf{A}\left(\mathbf{x}^{*}\right)}
\end{aligned}
$$

which is $(7.22)$.

(iv) Direct estimation of the second term on the right-hand side of (7.20), like in (iii), would yield a bound with a factor $\left\|\dot{\mathbf{e}}_{\mathbf{u}}\right\|_{\mathbf{A}\left(\mathbf{x}^{*}\right)}$, which cannot be 
controlled as required. The second term is therefore first rewritten, using the product rule of differentiation as

$$
\begin{aligned}
-\dot{\mathbf{e}}_{\mathbf{u}}^{T}\left(\mathbf{A}(\mathbf{x})-\mathbf{A}\left(\mathbf{x}^{*}\right)\right) \mathbf{u}^{*}= & -\frac{\mathrm{d}}{\mathrm{d} t}\left(\mathbf{e}_{\mathbf{u}}^{T}\left(\mathbf{A}(\mathbf{x})-\mathbf{A}\left(\mathbf{x}^{*}\right)\right) \mathbf{u}^{*}\right) \\
& +\mathbf{e}_{\mathbf{u}}^{T}\left(\mathbf{A}(\mathbf{x})-\mathbf{A}\left(\mathbf{x}^{*}\right)\right) \dot{\mathbf{u}}^{*}+\mathbf{e}_{\mathbf{u}}^{T} \frac{\mathrm{d}}{\mathrm{d} t}\left(\mathbf{A}(\mathbf{x})-\mathbf{A}\left(\mathbf{x}^{*}\right)\right) \mathbf{u}^{*}
\end{aligned}
$$

Among these terms, the first term on the second line is estimated via Lemmas 7.1 and 7.2, like in (iii), as

$$
\mathbf{e}_{\mathbf{u}}^{T}\left(\mathbf{A}(\mathbf{x})-\mathbf{A}\left(\mathbf{x}^{*}\right)\right) \dot{\mathbf{u}}^{*} \leq C\left\|\mathbf{e}_{\mathbf{u}}\right\|_{\mathbf{A}\left(\mathbf{x}^{*}\right)}\left\|\mathbf{e}_{\mathbf{x}}\right\|_{\mathbf{A}\left(\mathbf{x}^{*}\right)} .
$$

The last term is rewritten, using Lemma 7.1 and the Leibniz formula, as

$$
\begin{aligned}
\mathbf{w}^{T}\left(\frac{\mathrm{d}}{\mathrm{d} t}\left(\mathbf{A}(\mathbf{x})-\mathbf{A}\left(\mathbf{x}^{*}\right)\right)\right) \mathbf{z}= & \frac{\mathrm{d}}{\mathrm{d} t} \int_{0}^{1} \int_{\Gamma_{h}^{\theta}} \nabla_{\Gamma_{h}^{\theta}} w_{h}^{\theta} \cdot\left(D_{\Gamma_{h}^{\theta}} e_{x}^{\theta}\right) \nabla_{\Gamma_{h}^{\theta}} z_{h}^{\theta} \mathrm{d} \theta \\
= & \int_{0}^{1} \int_{\Gamma_{h}^{\theta}} \partial_{\Gamma_{h}^{\theta}}^{\bullet}\left(\nabla_{\Gamma_{h}^{\theta}} w_{h}^{\theta}\right) \cdot\left(D_{\Gamma_{h}^{\theta}} e_{x}^{\theta}\right) \nabla_{\Gamma_{h}^{\theta}} z_{h}^{\theta} \mathrm{d} \theta \\
& +\int_{0}^{1} \int_{\Gamma_{h}^{\theta}} \nabla_{\Gamma_{h}^{\theta}} w_{h}^{\theta} \cdot \partial_{\Gamma_{h}^{\theta}}^{\bullet}\left(D_{\Gamma_{h}^{\theta}} e_{x}^{\theta}\right) \nabla_{\Gamma_{h}^{\theta}} z_{h}^{\theta} \mathrm{d} \theta \\
& +\int_{0}^{1} \int_{\Gamma_{h}^{\theta}} \nabla_{\Gamma_{h}^{\theta}} w_{h}^{\theta} \cdot\left(D_{\Gamma_{h}^{\theta}} e_{x}^{\theta}\right) \partial_{\Gamma_{h}^{\theta}}^{\bullet}\left(\nabla_{\Gamma_{h}^{\theta}} z_{h}^{\theta}\right) \mathrm{d} \theta \\
& +\int_{0}^{1} \int_{\Gamma_{h}^{\theta}} \nabla_{\Gamma_{h}^{\theta}} w_{h}^{\theta} \cdot\left(D_{\Gamma_{h}^{\theta}} e_{x}^{\theta}\right) \nabla_{\Gamma_{h}^{\theta}} z_{h}^{\theta}\left(\nabla_{\Gamma_{h}^{\theta}} \cdot v_{\Gamma_{h}^{\theta}}\right) \mathrm{d} \theta \\
= & \int_{0}^{1}\left(J_{1}^{\theta}+J_{2}^{\theta}+J_{3}^{\theta}+J_{4}^{\theta}\right) \mathrm{d} \theta .
\end{aligned}
$$

Here, $v_{\Gamma_{h}^{\theta}}(\cdot, t)$ is the velocity of $\Gamma_{h}^{\theta}(t)$ (as a function of $t$ ), which is the finite element function in $S_{h}\left[\mathbf{x}^{*}(t)+\theta \mathbf{e}_{\mathbf{x}}(t)\right]$ with nodal vector $\dot{\mathbf{x}}^{*}(t)+\theta \dot{\mathbf{e}}_{\mathbf{x}}(t)=$ $\mathbf{v}^{*}(t)+\theta \mathbf{e}_{\mathbf{v}}(t)$. Related to this velocity, $\partial_{\Gamma_{h}^{\theta}}^{\bullet}$ denotes the material derivative on $\Gamma_{h}^{\theta}$. We denote by $w_{h}^{\theta}(\cdot, t)$ and $z_{h}^{\theta}(\cdot, t)$ the finite element functions on $\Gamma_{h}^{\theta}(t)$ with the time-independent nodal vectors $\mathbf{w}$ and $\mathbf{z}$, respectively. We thus have

$$
v_{\Gamma_{h}^{\theta}}=v_{h}^{*, \theta}+\theta e_{v}^{\theta}
$$

By using the identity (see [23, Lemma 2.6])

$$
\begin{aligned}
\partial_{\Gamma_{h}^{\theta}}^{\bullet}\left(\nabla_{\Gamma_{h}^{\theta}} w_{h}^{\theta}\right) & =\nabla_{\Gamma_{h}^{\theta}} \partial_{\Gamma_{h}^{\theta}}^{\bullet} w_{h}^{\theta}-\left(\nabla_{\Gamma_{h}^{\theta}} v_{\Gamma_{h}^{\theta}}-\nu_{h}^{\theta}\left(\nu_{h}^{\theta}\right)^{T}\left(\nabla_{\Gamma_{h}^{\theta}} v_{\Gamma_{h}^{\theta}}\right)^{T}\right) \nabla_{\Gamma_{h}^{\theta}} w_{h}^{\theta} \\
& =-\left(\nabla_{\Gamma_{h}^{\theta}} v_{\Gamma_{h}^{\theta}}-\nu_{h}^{\theta}\left(\nu_{h}^{\theta}\right)^{T}\left(\nabla_{\Gamma_{h}^{\theta}} v_{\Gamma_{h}^{\theta}}\right)^{T}\right) \nabla_{\Gamma_{h}^{\theta}} w_{h}^{\theta},
\end{aligned}
$$


where we have used the property $\partial_{\Gamma_{h}^{\theta}}^{\bullet} w_{h}^{\theta}=0$ in the last equality, we obtain

$$
\begin{aligned}
\left|J_{1}^{\theta}\right| & \leq \int_{0}^{1} \int_{\Gamma_{h}^{\theta}}\left|\left(\left(\nabla_{\Gamma_{h}^{\theta}} v_{\Gamma_{h}^{\theta}}-\nu_{h}^{\theta}\left(\nu_{h}^{\theta}\right)^{T}\left(\nabla_{\Gamma_{h}^{\theta}} v_{\Gamma_{h}^{\theta}}\right)^{T}\right) \nabla_{\Gamma_{h}^{\theta}} w_{h}^{\theta}\right) \cdot\left(D_{\Gamma_{h}^{\theta}} e_{x}^{\theta}\right) \nabla_{\Gamma_{h}^{\theta}} z_{h}^{\theta}\right| \mathrm{d} \theta \\
& \leq c \int_{0}^{1}\left\|\nabla_{\Gamma_{h}^{\theta}} w_{h}^{\theta}\right\|_{L^{2}\left(\Gamma_{h}^{\theta}\right)}\left\|\nabla_{\Gamma_{h}^{\theta}} v_{h}^{*, \theta}\right\|_{L^{\infty}\left(\Gamma_{h}^{\theta}\right)}\left\|D_{\Gamma_{h}^{\theta}} e_{x}^{\theta}\right\|_{L^{2}\left(\Gamma_{h}^{\theta}\right)}\left\|\nabla_{\Gamma_{h}^{\theta}} z_{h}^{\theta}\right\|_{L^{\infty}\left(\Gamma_{h}^{\theta}\right)} \mathrm{d} \theta \\
& +c \int_{0}^{1}\left\|\nabla_{\Gamma_{h}^{\theta}} w_{h}^{\theta}\right\|_{L^{2}\left(\Gamma_{h}^{\theta}\right)}\left\|\nabla_{\Gamma_{h}^{\theta}} e_{v}^{\theta}\right\|_{L^{2}\left(\Gamma_{h}^{\theta}\right)}\left\|D_{\Gamma_{h}^{\theta}} e_{x}^{\theta}\right\|_{L^{\infty}\left(\Gamma_{h}^{\theta}\right)}\left\|\nabla_{\Gamma_{h}^{\theta}} z_{h}^{\theta}\right\|_{L^{\infty}\left(\Gamma_{h}^{\theta}\right)} \mathrm{d} \theta \\
& \leq c\left\|\nabla_{\Gamma_{h}\left[\mathbf{x}^{*}\right]} w_{h}\right\|_{L^{2}\left(\Gamma_{h}\left[\mathbf{x}^{*}\right]\right)}\left\|D_{\Gamma_{h}\left[\mathbf{x}^{*}\right]} e_{h}\right\|_{L^{2}\left(\Gamma_{h}\left[\mathbf{x}^{*}\right]\right)}\left\|\nabla_{\Gamma_{h}\left[\mathbf{x}^{*}\right]} z_{h}\right\|_{L^{\infty}\left(\Gamma_{h}\left[\mathbf{x}^{*}\right]\right)} \\
& +c\left\|\nabla_{\Gamma_{h}\left[\mathbf{x}^{*}\right]} w_{h}\right\|_{L^{2}\left(\Gamma_{h}\left[\mathbf{x}^{*}\right]\right)}\left\|\nabla_{\Gamma_{h}\left[\mathbf{x}^{*}\right]} e_{v}\right\|_{L^{2}\left(\Gamma_{h}\left[\mathbf{x}^{*}\right]\right)}\left\|\nabla_{\Gamma_{h}\left[\mathbf{x}^{*}\right]} z_{h}\right\|_{L^{\infty}\left(\Gamma_{h}\left[\mathbf{x}^{*}\right]\right)} \\
& \leq c\|\mathbf{w}\|_{\mathbf{A}\left(\mathbf{x}^{*}\right)}\left(\left\|\mathbf{e}_{\mathbf{x}}\right\|_{\mathbf{A}\left(\mathbf{x}^{*}\right)}+\left\|\mathbf{e}_{\mathbf{v}}\right\|_{\mathbf{A}\left(\mathbf{x}^{*}\right)}\right)\left\|z_{h}\right\|_{W^{1, \infty}\left(\Gamma_{h}\left[\mathbf{x}^{*}\right]\right)},
\end{aligned}
$$

where $z_{h}=z_{h}^{0}$ and where we have used the decomposition (7.25), Lemma 7.2, and the bounds (7.21). Similarly, we obtain

$$
\left|J_{2}^{\theta}\right|+\left|J_{3}^{\theta}\right|+\left|J_{4}^{\theta}\right| \leq c\|\mathbf{w}\|_{\mathbf{A}\left(\mathbf{x}^{*}\right)}\left(\left\|\mathbf{e}_{\mathbf{x}}\right\|_{\mathbf{A}\left(\mathbf{x}^{*}\right)}+\left\|\mathbf{e}_{\mathbf{v}}\right\|_{\mathbf{A}\left(\mathbf{x}^{*}\right)}\right)\left\|z_{h}\right\|_{W^{1, \infty}\left(\Gamma_{h}\left[\mathbf{x}^{*}\right]\right)}
$$

where for the estimate for $J_{2}^{\theta}$ we used the equality analogous to (7.26):

$$
\begin{gathered}
\partial_{\Gamma_{h}^{\theta}}^{\bullet}\left(D_{\Gamma_{h}^{\theta}} e_{x}^{\theta}\right)=\partial_{\Gamma_{h}^{\theta}}^{\bullet}\left(\operatorname{tr}\left(\nabla_{\Gamma_{h}^{\theta}} e_{x}^{\theta}\right)-\left(\nabla_{\Gamma_{h}^{\theta}} e_{x}^{\theta}+\left(\nabla_{\Gamma_{h}^{\theta}} e_{x}^{\theta}\right)^{T}\right)\right) \\
=D_{\Gamma_{h}^{\theta}}\left(\partial_{\Gamma_{h}^{\theta}}^{\bullet} e_{x}^{\theta}\right)+\operatorname{tr}\left(\bar{E}^{\theta}\right)-\left(\bar{E}^{\theta}+\left(\bar{E}^{\theta}\right)^{T}\right),
\end{gathered}
$$

with $\bar{E}^{\theta}=-\left(\nabla_{\Gamma_{h}^{\theta}} v_{\Gamma_{h}^{\theta}}-\nu_{h}^{\theta}\left(\nu_{h}^{\theta}\right)^{T}\left(\nabla_{\Gamma_{h}^{\theta}} v_{\Gamma_{h}^{\theta}}\right)^{T}\right) \nabla_{\Gamma_{h}^{\theta}} e_{x}^{\theta}$, as follows from [23, Lemma 2.6] and the definition of the first order linear differential operator $D_{\Gamma_{h}^{\theta}}$.

Further using $\partial_{\Gamma_{h}^{\theta}}^{\bullet} e_{x}^{\theta}=e_{v}^{\theta}$ (since for the nodal vectors we have $\dot{\mathbf{e}}_{\mathbf{x}}=\mathbf{e}_{\mathbf{v}}$ ), we altogether obtain the bound

$$
\begin{aligned}
& -\dot{\mathbf{e}}_{\mathbf{u}}^{T}\left(\mathbf{A}(\mathbf{x})-\mathbf{A}\left(\mathbf{x}^{*}\right)\right) \mathbf{u}^{*} \\
& \leq-\frac{\mathrm{d}}{\mathrm{d} t}\left(\mathbf{e}_{\mathbf{u}}^{T}\left(\mathbf{A}(\mathbf{x})-\mathbf{A}\left(\mathbf{x}^{*}\right)\right) \mathbf{u}^{*}\right)+c\left\|\mathbf{e}_{\mathbf{u}}\right\|_{\mathbf{A}\left(\mathbf{x}^{*}\right)}\left(\left\|\mathbf{e}_{\mathbf{v}}\right\|_{\mathbf{K}\left(\mathbf{x}^{*}\right)}+\left\|\mathbf{e}_{\mathbf{x}}\right\|_{\mathbf{K}\left(\mathbf{x}^{*}\right)}\right) .
\end{aligned}
$$

(v) The term containing the nonlinearity $\mathbf{f}$ can be written as

$$
\dot{\mathbf{e}}_{\mathbf{u}}^{T}\left(\mathbf{f}(\mathbf{x}, \mathbf{u})-\mathbf{f}\left(\mathbf{x}^{*}, \mathbf{u}^{*}\right)\right)=\int_{\Gamma_{h}^{1}} f\left(u_{h}, \nabla_{\Gamma_{h}^{1}} u_{h}\right) \cdot \dot{e}_{u}^{1}-\int_{\Gamma_{h}^{0}} f\left(u_{h}^{*}, \nabla_{\Gamma_{h}^{0}} u_{h}^{*}\right) \cdot \dot{e}_{u}^{0},
$$

where the function $f$ is given by the right-hand side of $(2.6)$ for $u=(\nu, H)$; $f: \mathbb{R}^{4} \times \mathbb{R}^{3 \times 4} \rightarrow \mathbb{R}^{4}$ is smooth and therefore locally Lipschitz continuous.

We estimate this term in the following way. Using the abbreviation

$$
u_{\Gamma_{h}^{\theta}}:=u_{h}^{*, \theta}+\theta e_{u}^{\theta}=\sum_{j=1}^{N}\left(u_{j}^{*}+\theta\left(\mathbf{e}_{\mathbf{u}}\right)_{j}\right) \phi_{j}\left[\mathbf{x}^{*}+\theta \mathbf{e}_{\mathbf{x}}\right],
$$

we have

$$
\dot{\mathbf{e}}_{\mathbf{u}}^{T}\left(\mathbf{f}(\mathbf{x}, \mathbf{u})-\mathbf{f}\left(\mathbf{x}^{*}, \mathbf{u}^{*}\right)\right)=\int_{0}^{1} \frac{\mathrm{d}}{\mathrm{d} \theta} \int_{\Gamma_{h}^{\theta}} f\left(u_{\Gamma_{h}^{\theta}}, \nabla_{\Gamma_{h}^{\theta}} u_{\Gamma_{h}^{\theta}}\right) \cdot \dot{e}_{u}^{\theta} \mathrm{d} \theta
$$


Here we apply the Leibniz formula, noting that $e_{x}^{\theta}$ is the velocity of the surface $\Gamma_{h}^{\theta}$ considered as a function of $\theta$ and that we have the vanishing material derivative

$$
\partial_{\theta} \dot{e}_{u}^{\theta}=\sum_{j=1}^{N} \frac{\mathrm{d}}{\mathrm{d} \theta}\left(\dot{\mathbf{e}}_{\mathbf{u}}\right)_{j} \phi_{j}\left[\mathbf{x}^{*}+\theta \mathbf{e}_{\mathbf{x}}\right]=0 .
$$

So we obtain

$$
\begin{aligned}
& \dot{\mathbf{e}}_{\mathbf{u}}^{T}\left(\mathbf{f}(\mathbf{x}, \mathbf{u})-\mathbf{f}\left(\mathbf{x}^{*}, \mathbf{u}^{*}\right)\right) \\
& =\int_{0}^{1} \int_{\Gamma_{h}^{\theta}}\left(\partial_{\theta}^{\bullet}\left(f\left(u_{\Gamma_{h}^{\theta}}, \nabla_{\Gamma_{h}^{\theta}} u_{\Gamma_{h}^{\theta}}\right)\right) \cdot \dot{e}_{u}^{\theta}+f\left(u_{\Gamma_{h}^{\theta}}, \nabla_{\Gamma_{h}^{\theta}} u_{\Gamma_{h}^{\theta}}\right) \cdot \dot{e}_{u}^{\theta}\left(\nabla_{\Gamma_{h}^{\theta}} \cdot e_{x}^{\theta}\right)\right) \mathrm{d} \theta .
\end{aligned}
$$

Here we proceed further using the chain rule

$$
\begin{aligned}
\partial_{\theta}^{\bullet} f\left(u_{\Gamma_{h}^{\theta}}, \nabla_{\Gamma_{h}^{\theta}} u_{\Gamma_{h}^{\theta}}\right) & =\partial_{1} f\left(u_{\Gamma_{h}^{\theta}}, \nabla_{\Gamma_{h}^{\theta}} u_{\Gamma_{h}^{\theta}}\right) \partial_{\theta}^{\bullet} u_{\Gamma_{h}^{\theta}} \\
& +\partial_{2} f\left(u_{\Gamma_{h}^{\theta}}, \nabla_{\Gamma_{h}^{\theta}} u_{\Gamma_{h}^{\theta}}\right) \partial_{\theta}^{\bullet}\left(\nabla_{\Gamma_{h}^{\theta}} u_{\Gamma_{h}^{\theta}}\right)
\end{aligned}
$$

and observe the following: by the $W^{1, \infty}$ bound for the exact solution $u^{*}$, and for $e_{u}$ in (7.21) (and hence for $e_{u}^{\theta}$ by Lemma 7.2), we have on recalling (7.28) that $u_{\Gamma_{h}^{\theta}}$ and its gradient take values in a bounded set. Since $f$ is smooth, we therefore have

$$
\left\|\partial_{i} f\left(u_{\Gamma_{h}^{\theta}}, \nabla_{\Gamma_{h}^{\theta}} u_{\Gamma_{h}^{\theta}}\right)\right\|_{L^{\infty}\left(\Gamma_{h}^{\theta}\right)} \leq C, \quad i=1,2 .
$$

From $u_{\Gamma_{h}^{\theta}}=u_{h}^{*}+\theta e_{u}^{\theta}$ and the identity $\partial_{\theta}^{\bullet} e_{u}^{\theta}=0$ (obtained by the same argument as for $\dot{e}_{u}^{\theta}$ above) we note

$$
\partial_{\theta}^{\bullet} u_{\Gamma_{h}^{\theta}}=e_{u}^{\theta} .
$$

We further use the relation, see [23, Lemma 2.6],

$$
\partial_{\theta}^{\bullet}\left(\nabla_{\Gamma_{h}^{\theta}} u_{\Gamma_{h}^{\theta}}\right)=\nabla_{\Gamma_{h}^{\theta}}\left(\partial_{\theta}^{\bullet} u_{\Gamma_{h}^{\theta}}\right)-\left(\nabla_{\Gamma_{h}^{\theta}} e_{x}^{\theta}-\nu_{h}^{\theta}\left(\nu_{h}^{\theta}\right)^{T}\left(\nabla_{\Gamma_{h}^{\theta}} e_{x}^{\theta}\right)^{T}\right) \nabla_{\Gamma_{h}^{\theta}} u_{\Gamma_{h}^{\theta}} .
$$

We then have, on inserting (7.28) and using once again Lemma 7.2 and the bounds in (7.21),

$$
\begin{aligned}
& \quad \dot{\mathbf{e}}_{\mathbf{u}}^{T}\left(\mathbf{f}(\mathbf{x}, \mathbf{u})-\mathbf{f}\left(\mathbf{x}^{*}, \mathbf{u}^{*}\right)\right) \\
& =\int_{0}^{1} \int_{\Gamma_{h}^{\theta}} \dot{e}_{u}^{\theta}\left(f\left(u_{\Gamma_{h}^{\theta}}, \nabla_{\Gamma_{h}^{\theta}} u_{\Gamma_{h}^{\theta}}\right) \cdot\left(\nabla_{\Gamma_{h}^{\theta}} \cdot e_{x}^{\theta}\right)+\partial_{1} f\left(u_{\Gamma_{h}^{\theta}}, \nabla_{\Gamma_{h}^{\theta}} u_{\Gamma_{h}^{\theta}}\right) e_{u}^{\theta}\right. \\
& \left.\quad+\partial_{2} f\left(u_{\Gamma_{h}^{\theta}}, \nabla_{\Gamma_{h}^{\theta}} u_{\Gamma_{h}^{\theta}}\right)\left(\nabla_{\Gamma_{h}^{\theta}} e_{u}^{\theta}-\left(\nabla_{\Gamma_{h}^{\theta}} e_{x}^{\theta}-\nu_{h}^{\theta}\left(\nu_{h}^{\theta}\right)^{T}\left(\nabla_{\Gamma_{h}^{\theta}} e_{x}^{\theta}\right)^{T}\right) \nabla_{\Gamma_{h}^{\theta}} u_{\Gamma_{h}^{\theta}}\right)\right) \mathrm{d} \theta \\
& \leq c\left\|\dot{e}_{u}\right\|_{L^{2}\left(\Gamma_{h}\left[\mathbf{x}^{*}\right]\right)}\left(\left\|e_{u}\right\|_{L^{2}\left(\Gamma_{h}\left[\mathbf{x}^{*}\right]\right)}\right. \\
& \left.\quad+\left\|\nabla_{\left.\Gamma_{h}\left[\mathbf{x}^{*}\right]\right)} e_{u}\right\|_{L^{2}\left(\Gamma_{h}\left[\mathbf{x}^{*}\right]\right)}+\left\|\nabla_{\left.\Gamma_{h}\left[\mathbf{x}^{*}\right]\right)} e_{x}\right\|_{L^{2}\left(\Gamma_{h}\left[\mathbf{x}^{*}\right]\right)}\left\|\nabla_{\left.\Gamma_{h}\left[\mathbf{x}^{*}\right]\right)} u_{h}^{*}\right\|_{L^{\infty}\left(\Gamma_{h}\left[\mathbf{x}^{*}\right]\right)}\right) \\
& \leq c\left\|\dot{\mathbf{e}}_{\mathbf{u}}\right\|_{\mathbf{M}\left(\mathbf{x}^{*}\right)}\left(\left\|\mathbf{e}_{\mathbf{u}}\right\|_{\mathbf{K}\left(\mathbf{x}^{*}\right)}+\left\|\mathbf{e}_{\mathbf{x}}\right\|_{\mathbf{A}\left(\mathbf{x}^{*}\right)}\right) .
\end{aligned}
$$


(vi) The defect term is bounded using the Cauchy-Schwarz inequality:

$$
-\dot{\mathbf{e}}_{\mathbf{u}}^{T} \mathbf{M}\left(\mathbf{x}^{*}\right) \mathbf{d}_{\mathbf{u}} \leq\left\|\dot{\mathbf{e}}_{\mathbf{u}}\right\|_{\mathbf{M}\left(\mathbf{x}^{*}\right)}\left\|\mathbf{d}_{\mathbf{u}}\right\|_{\mathbf{M}\left(\mathbf{x}^{*}\right)} .
$$

The combination of the above inequalities and the norm equivalence (7.7) yield the bound

$$
\begin{aligned}
& \left\|\dot{\mathbf{e}}_{\mathbf{u}}\right\|_{\mathbf{M}(\mathbf{x})}^{2}+\frac{1}{2} \frac{\mathrm{d}}{\mathrm{d} t}\left\|\mathbf{e}_{\mathbf{u}}\right\|_{\mathbf{A}(\mathbf{x})}^{2} \\
& \leq c\left\|\mathbf{e}_{\mathbf{u}}\right\|_{\mathbf{A}\left(\mathbf{x}^{*}\right)}^{2}+c\left\|\dot{\mathbf{e}}_{\mathbf{u}}\right\|_{\mathbf{M}\left(\mathbf{x}^{*}\right)}\left\|\mathbf{e}_{\mathbf{x}}\right\|_{\mathbf{K}\left(\mathbf{x}^{*}\right)} \\
& \quad+c\left\|\mathbf{e}_{\mathbf{u}}\right\|_{\mathbf{A}\left(\mathbf{x}^{*}\right)}\left(\left\|\mathbf{e}_{\mathbf{v}}\right\|_{\mathbf{K}\left(\mathbf{x}^{*}\right)}+\left\|\mathbf{e}_{\mathbf{x}}\right\|_{\mathbf{K}\left(\mathbf{x}^{*}\right)}\right) \\
& \quad-\frac{\mathrm{d}}{\mathrm{d} t}\left(\mathbf{e}_{\mathbf{u}}^{T}\left(\mathbf{A}(\mathbf{x})-\mathbf{A}\left(\mathbf{x}^{*}\right)\right) \mathbf{u}^{*}\right) \\
& \quad+c\left\|\dot{\mathbf{e}}_{\mathbf{u}}\right\|_{\mathbf{M}\left(\mathbf{x}^{*}\right)}\left(\left\|\mathbf{e}_{\mathbf{u}}\right\|_{\mathbf{K}\left(\mathbf{x}^{*}\right)}+\left\|\mathbf{e}_{\mathbf{x}}\right\|_{\mathbf{K}\left(\mathbf{x}^{*}\right)}\right) \\
& \quad+\left\|\dot{\mathbf{e}}_{\mathbf{u}}\right\|_{\mathbf{M}\left(\mathbf{x}^{*}\right)}\left\|\mathbf{d}_{\mathbf{u}}\right\|_{\mathbf{M}\left(\mathbf{x}^{*}\right)} .
\end{aligned}
$$

Here we note for the first term on the left-hand side that, by (7.11),

$$
\frac{1}{2} \frac{\mathrm{d}}{\mathrm{d} t}\left\|\mathbf{e}_{\mathbf{u}}\right\|_{\mathbf{M}(\mathbf{x})}^{2}=\mathbf{e}_{\mathbf{u}}^{T} \mathbf{M}(\mathbf{x}) \dot{\mathbf{e}}_{\mathbf{u}}+\frac{1}{2} \mathbf{e}_{\mathbf{u}}^{T} \frac{\mathrm{d}}{\mathrm{d} t} \mathbf{M}(\mathbf{x}) \mathbf{e}_{\mathbf{u}} \leq \frac{1}{2}\left\|\dot{\mathbf{e}}_{\mathbf{u}}\right\|_{\mathbf{M}(\mathbf{x})}^{2}+c\left\|\mathbf{e}_{\mathbf{u}}\right\|_{\mathbf{M}(\mathbf{x})}^{2}
$$

Estimating further, using the norm equivalence (7.7) and Young's inequality and absorptions (with $h \leq h_{0}$ for a sufficiently small $h_{0}$ ) into $\left\|\dot{\mathbf{e}}_{\mathbf{u}}\right\|_{\mathbf{M}\left(\mathbf{x}^{*}\right)}^{2}$, we obtain the following estimate:

$$
\begin{aligned}
& \frac{1}{2} \frac{\mathrm{d}}{\mathrm{d} t}\left\|\mathbf{e}_{\mathbf{u}}\right\|_{\mathbf{M}(\mathbf{x})}^{2}+\frac{1}{2} \frac{\mathrm{d}}{\mathrm{d} t}\left\|\mathbf{e}_{\mathbf{u}}\right\|_{\mathbf{A}(\mathbf{x})}^{2} \\
& \leq c\left\|\mathbf{e}_{\mathbf{x}}\right\|_{\mathbf{K}\left(\mathbf{x}^{*}\right)}^{2}+c\left\|\mathbf{e}_{\mathbf{v}}\right\|_{\mathbf{K}\left(\mathbf{x}^{*}\right)}^{2}+c\left\|\mathbf{e}_{\mathbf{u}}\right\|_{\mathbf{K}\left(\mathbf{x}^{*}\right)}^{2} \\
& \quad-\frac{\mathrm{d}}{\mathrm{d} t}\left(\mathbf{e}_{\mathbf{u}}^{T}\left(\mathbf{A}(\mathbf{x})-\mathbf{A}\left(\mathbf{x}^{*}\right)\right) \mathbf{u}^{*}\right) \\
& \quad+c\left\|\mathbf{d}_{\mathbf{u}}\right\|_{\mathbf{M}\left(\mathbf{x}^{*}\right)}^{2} .
\end{aligned}
$$

After integration in time, using the above estimates together with Young's inequality, assumption (7.21) and the norm equivalence (7.7), and recalling 
that $\mathbf{M}\left(\mathrm{x}^{*}\right)+\mathbf{A}\left(\mathrm{x}^{*}\right)=\mathbf{K}\left(\mathrm{x}^{*}\right)$, we obtain

$$
\begin{aligned}
& \frac{1}{2}\left\|\mathbf{e}_{\mathbf{u}}(t)\right\|_{\mathbf{K}\left(\mathbf{x}^{*}(t)\right)}^{2} \leq \frac{1}{2}\left\|\mathbf{e}_{\mathbf{u}}(0)\right\|_{\mathbf{K}\left(\mathbf{x}^{*}(0)\right)}^{2} \\
& \quad+c \int_{0}^{t}\left(\left\|\mathbf{e}_{\mathbf{v}}(s)\right\|_{\mathbf{K}\left(\mathbf{x}^{*}(s)\right)}^{2}+\left\|\mathbf{e}_{\mathbf{u}}(s)\right\|_{\mathbf{K}\left(\mathbf{x}^{*}(s)\right)}^{2}+\left\|\mathbf{e}_{\mathbf{x}}(s)\right\|_{\mathbf{K}\left(\mathbf{x}^{*}(s)\right)}^{2}\right) \mathrm{d} s \\
& \quad-\mathbf{e}_{\mathbf{u}}(t)^{T}\left(\mathbf{A}(\mathbf{x}(t))-\mathbf{A}\left(\mathbf{x}^{*}(t)\right)\right) \mathbf{u}^{*}(t) \\
& \quad+c \int_{0}^{t}\left\|\mathbf{d}_{\mathbf{u}}(s)\right\|_{\mathbf{M}\left(\mathbf{x}^{*}(s)\right)}^{2} \mathrm{~d} s \\
& \leq \frac{1}{2}\left\|\mathbf{e}_{\mathbf{u}}(0)\right\|_{\mathbf{K}\left(\mathbf{x}^{*}(0)\right)}^{2} \\
& \quad+c \int_{0}^{t}\left(\left\|\mathbf{e}_{\mathbf{x}}(s)\right\|_{\mathbf{K}\left(\mathbf{x}^{*}(s)\right)}^{2}+\left\|\mathbf{e}_{\mathbf{v}}(s)\right\|_{\mathbf{K}\left(\mathbf{x}^{*}(s)\right)}^{2}+\left\|\mathbf{e}_{\mathbf{u}}(s)\right\|_{\mathbf{K}\left(\mathbf{x}^{*}(s)\right)}^{2}\right) \mathrm{d} s \\
& \quad+\frac{1}{6}\left\|\mathbf{e}_{\mathbf{u}}(t)\right\|_{\mathbf{A}\left(\mathbf{x}^{*}(t)\right)}^{2}+c\left\|\mathbf{e}_{\mathbf{x}}(t)\right\|_{\mathbf{K}\left(\mathbf{x}^{*}(t)\right)}^{2} \\
& \quad+c \int_{0}^{t}\left\|\mathbf{d}_{\mathbf{u}}(s)\right\|_{\mathbf{M}\left(\mathbf{x}^{*}(s)\right)}^{2} \mathrm{~d} s,
\end{aligned}
$$

where the term involving the stiffness matrices was estimated analogously as (iii), and with the help of the Young inequality.

Absorption of the term containing $\mathbf{e}_{\mathbf{u}}(t)$ (in the case of a sufficiently small h) finally yields

$$
\begin{aligned}
\left\|\mathbf{e}_{\mathbf{u}}(t)\right\|_{\mathbf{K}\left(\mathbf{x}^{*}(t)\right)}^{2} & \leq c \int_{0}^{t}\left(\left\|\mathbf{e}_{\mathbf{x}}(s)\right\|_{\mathbf{K}\left(\mathbf{x}^{*}(s)\right)}^{2}+\left\|\mathbf{e}_{\mathbf{v}}(s)\right\|_{\mathbf{K}\left(\mathbf{x}^{*}(s)\right)}^{2}+\left\|\mathbf{e}_{\mathbf{u}}(s)\right\|_{\mathbf{K}\left(\mathbf{x}^{*}(s)\right)}^{2}\right) \mathrm{d} s \\
& +c\left\|\mathbf{e}_{\mathbf{x}}(t)\right\|_{\mathbf{K}\left(\mathbf{x}^{*}(t)\right)}^{2}+c\left\|\mathbf{e}_{\mathbf{u}}(0)\right\|_{\mathbf{K}\left(\mathbf{x}^{*}(0)\right)}^{2}+c \int_{0}^{t}\left\|\mathbf{d}_{\mathbf{u}}(s)\right\|_{\mathbf{M}\left(\mathbf{x}^{*}(s)\right)}^{2} \mathrm{~d} s .
\end{aligned}
$$

(B) Estimates for the velocity equation: Testing (7.19) with $\mathbf{e}_{\mathbf{v}}$ and using Lemma 7.1, the Cauchy-Schwarz and Young inequalities, and the norm equivalence (7.7) yields the bound

$$
\frac{1}{2}\left\|\mathbf{e}_{\mathbf{v}}\right\|_{\mathbf{K}\left(\mathbf{x}^{*}\right)}^{2} \leq c\left\|\mathbf{e}_{\mathbf{x}}\right\|_{\mathbf{K}\left(\mathbf{x}^{*}\right)}^{2}+\mathbf{e}_{\mathbf{v}}^{T}\left(\mathbf{g}(\mathbf{x}, \mathbf{u})-\mathbf{g}\left(\mathbf{x}^{*}, \mathbf{u}^{*}\right)\right)+c\left\|\mathbf{d}_{\mathbf{v}}\right\|_{\star, \mathbf{x}^{*}}^{2} .
$$

The term with the nonlinearity $\mathbf{g}$ is first rewritten as

$$
\begin{aligned}
& \mathbf{e}_{\mathbf{v}}^{T}\left(\mathbf{g}(\mathbf{x}, \mathbf{u})-\mathbf{g}\left(\mathbf{x}^{*}, \mathbf{u}^{*}\right)\right) \\
& \quad=\int_{\Gamma_{h}^{1}} g\left(u_{h}\right) e_{v}^{1}-\int_{\Gamma_{h}^{0}} g\left(u_{h}^{*}\right) e_{v}^{0} \\
& \quad+\int_{\Gamma_{h}^{1}} G\left(u_{h}, \nabla_{\Gamma_{h}^{1}} u_{h}\right) \cdot \nabla_{\Gamma_{h}^{1}} e_{v}^{1}-\int_{\Gamma_{h}^{0}} G\left(u_{h}^{*}, \nabla_{\Gamma_{h}^{0}} u_{h}^{*}\right) \cdot \nabla_{\Gamma_{h}^{0}} e_{v}^{0},
\end{aligned}
$$

where the functions $g: \mathbb{R}^{4} \rightarrow \mathbb{R}^{3}$ and $G: \mathbb{R}^{4} \times \mathbb{R}^{3 \times 4} \rightarrow \mathbb{R}^{3 \times 3}$ are smooth and hence locally Lipschitz-continuous functions as given by (2.6). 
We now use the same argument as in (v) for the nonlinearity in the equation for the dynamic variables $u$. Using formula (7.28) and the Leibniz formula, recalling that $e_{x}^{\theta}$ is the velocity of $\Gamma_{h}^{\theta}$ as a function of $\theta$, and also using that $\partial_{\theta}^{\bullet} e_{v}^{\theta}=0$, we again obtain

$$
\begin{aligned}
& \mathbf{e}_{\mathbf{v}}^{T}\left(\mathbf{g}(\mathbf{x}, \mathbf{u})-\mathbf{g}\left(\mathbf{x}^{*}, \mathbf{u}^{*}\right)\right) \\
= & \int_{0}^{1} \int_{\Gamma_{h}^{\theta}} \partial_{\theta}^{\bullet}\left(g\left(u_{\Gamma_{h}^{\theta}}\right)\right) \cdot e_{v}^{\theta} \mathrm{d} \theta+\int_{0}^{1} \int_{\Gamma_{h}^{\theta}} \partial_{\theta}^{\bullet}\left(G\left(u_{\Gamma_{h}^{\theta}}, \nabla_{\Gamma_{h}^{\theta}} u_{\Gamma_{h}^{\theta}}\right)\right) \cdot \nabla_{\Gamma_{h}^{\theta}} e_{v}^{\theta} \mathrm{d} \theta \\
& +\int_{0}^{1} \int_{\Gamma_{h}^{\theta}} G\left(u_{\Gamma_{h}^{\theta}}, \nabla_{\Gamma_{h}^{\theta}} u_{\Gamma_{h}^{\theta}}\right) \cdot \partial_{\theta}^{\bullet}\left(\nabla_{\Gamma_{h}^{\theta}} e_{v}^{\theta}\right) \mathrm{d} \theta \\
& +\int_{0}^{1} \int_{\Gamma_{h}^{\theta}}\left(g\left(u_{\Gamma_{h}^{\theta}}\right) \cdot e_{v}^{\theta}+G\left(u_{\Gamma_{h}^{\theta}}, \nabla_{\Gamma_{h}^{\theta}} u_{\Gamma_{h}^{\theta}}\right) \cdot \nabla_{\Gamma_{h}^{\theta}} e_{v}^{\theta}\right)\left(\nabla_{\Gamma_{h}^{\theta}} \cdot e_{x}^{\theta}\right) \mathrm{d} \theta .
\end{aligned}
$$

Here we use the chain rule

$$
\begin{aligned}
\partial_{\theta}^{\bullet} g\left(u_{\Gamma_{h}^{\theta}}\right)= & \partial_{1} g\left(u_{\Gamma_{h}^{\theta}}\right) \partial_{\theta}^{\bullet} u_{\Gamma_{h}^{\theta}} \\
\partial_{\theta}^{\bullet} G\left(u_{\Gamma_{h}^{\theta}}, \nabla_{\Gamma_{h}^{\theta}} u_{\Gamma_{h}^{\theta}}\right) & =\partial_{1} G\left(u_{\Gamma_{h}^{\theta}}, \nabla_{\Gamma_{h}^{\theta}} u_{\Gamma_{h}^{\theta}}\right) \partial_{\theta}^{\bullet} u_{\Gamma_{h}^{\theta}} \\
& +\partial_{2} G\left(u_{\Gamma_{h}^{\theta}}, \nabla_{\Gamma_{h}^{\theta}} u_{\Gamma_{h}^{\theta}}\right) \partial_{\theta}^{\bullet}\left(\nabla_{\Gamma_{h}^{\theta}} u_{\Gamma_{h}^{\theta}}\right) .
\end{aligned}
$$

By the $W^{1, \infty}$ bound for $u_{h}^{*}$ and $e_{u}$ (by (7.21)), and hence for $e_{u}^{\theta}$ by Lemma 7.2, the arguments of $g$ and $G$ take values in a bounded set. By the smoothness of both functions, we therefore have

$$
\left\|\partial_{1} g\left(u_{\Gamma_{h}^{\theta}}\right)\right\|_{L^{\infty}\left(\Gamma_{h}^{\theta}\right)} \leq C \quad \text { and } \quad\left\|\partial_{i} G\left(u_{\Gamma_{h}^{\theta}} \nabla_{\Gamma_{h}^{\theta}} u_{\Gamma_{h}^{\theta}}\right)\right\|_{L^{\infty}\left(\Gamma_{h}^{\theta}\right)} \leq C, \quad i=1,2
$$

Using $\partial_{\theta}^{\bullet} u_{\Gamma_{h}^{\theta}}=e_{u}^{\theta}$ and $\partial_{\theta}^{\bullet} e_{v}^{\theta}=0$, the relations (7.29), (7.27), (7.28), and using once again Lemma 7.2 and the bounds (7.21), we obtain

$$
\begin{aligned}
& \mathbf{e}_{\mathbf{v}}^{T}\left(\mathbf{g}(\mathbf{x}, \mathbf{u})-\mathbf{g}\left(\mathbf{x}^{*}, \mathbf{u}^{*}\right)\right) \\
& =\int_{0}^{1} \int_{\Gamma_{h}^{\theta}} \partial_{1} g\left(u_{\Gamma_{h}^{\theta}}\right) e_{u}^{\theta} \cdot e_{v}^{\theta} \mathrm{d} \theta+\int_{0}^{1} \int_{\Gamma_{h}^{\theta}}\left(\partial_{1} G\left(u_{\Gamma_{h}^{\theta}}, \nabla_{\Gamma_{h}^{\theta}} u_{\Gamma_{h}^{\theta}}\right) e_{u}^{\theta}\right. \\
& +\partial_{2} G\left(u_{\Gamma_{h}^{\theta}}, \nabla_{\Gamma_{h}^{\theta}} u_{\Gamma_{h}^{\theta}}\right)\left(\nabla_{\Gamma_{h}^{\theta}} e_{u}^{\theta}-\left(\nabla_{\Gamma_{h}^{\theta}} e_{x}^{\theta}-\nu_{h}^{\theta}\left(\nu_{h}^{\theta}\right)^{T}\left(\nabla_{\Gamma_{h}^{\theta}} e_{x}^{\theta}\right)^{T}\right) \nabla_{\Gamma_{h}^{\theta}} u_{\Gamma_{h}^{\theta}}\right) \cdot \nabla_{\Gamma_{h}^{\theta}} e_{v}^{\theta} \mathrm{d} \theta \\
& \quad+\int_{0}^{1} \int_{\Gamma_{h}^{\theta}} G\left(u_{\Gamma_{h}^{\theta}}, \nabla_{\Gamma_{h}^{\theta}} u_{\Gamma_{h}^{\theta}}\right) \cdot\left(-\left(\nabla_{\Gamma_{h}^{\theta}} e_{x}^{\theta}-\nu_{h}^{\theta}\left(\nu_{h}^{\theta}\right)^{T}\left(\nabla_{\Gamma_{h}^{\theta}} e_{x}^{\theta}\right)^{T}\right) \nabla_{\Gamma_{h}^{\theta}} e_{v}^{\theta}\right) \mathrm{d} \theta \\
& \quad+\int_{0}^{1} \int_{\Gamma_{h}^{\theta}}\left(g\left(u_{\Gamma_{h}^{\theta}}\right) \cdot e_{v}^{\theta}+G\left(u_{\Gamma_{h}^{\theta}}, \nabla_{\Gamma_{h}^{\theta}} u_{\Gamma_{h}^{\theta}}\right) \cdot \nabla_{\Gamma_{h}^{\theta}} e_{v}^{\theta}\right)\left(\nabla_{\Gamma_{h}^{\theta}} \cdot e_{x}^{\theta}\right) \mathrm{d} \theta
\end{aligned}
$$


so that

$$
\begin{aligned}
& \mathbf{e}_{\mathbf{v}}^{T}\left(\mathbf{g}(\mathbf{x}, \mathbf{u})-\mathbf{g}\left(\mathbf{x}^{*}, \mathbf{u}^{*}\right)\right) \\
& \leq c\left\|e_{u}\right\|_{L^{2}\left(\Gamma_{h}\left[\mathbf{x}^{*}\right]\right)}\left\|e_{v}\right\|_{L^{2}\left(\Gamma_{h}\left[\mathbf{x}^{*}\right]\right)} \\
& \quad+c\left\|\nabla_{\Gamma_{h}\left[\mathbf{x}^{*}\right]} e_{v}\right\|_{L^{2}\left(\Gamma_{h}\left[\mathbf{x}^{*}\right]\right)}\left(\left\|e_{u}\right\|_{L^{2}\left(\Gamma_{h}\left[\mathbf{x}^{*}\right]\right)}+\left\|\nabla_{\Gamma_{h}\left[\mathbf{x}^{*}\right]} e_{u}\right\|_{L^{2}\left(\Gamma_{h}\left[\mathbf{x}^{*}\right]\right)}\right. \\
& \left.\quad+\left\|\nabla_{\Gamma_{h}\left[\mathbf{x}^{*}\right]} e_{x}\right\|_{L^{2}\left(\Gamma_{h}\left[\mathbf{x}^{*}\right]\right)}\left\|\nabla_{\Gamma_{h}\left[\mathbf{x}^{*}\right]} u_{h}^{*}\right\|_{L^{\infty}\left(\Gamma_{h}\left[\mathbf{x}^{*}\right]\right)}+\left\|\nabla_{\Gamma_{h}\left[\mathbf{x}^{*}\right]} e_{x}\right\|_{L^{2}\left(\Gamma_{h}\left[\mathbf{x}^{*}\right]\right)}\right) \\
& \quad+c\left\|e_{v}\right\|_{H^{1}\left(\Gamma_{h}\left[\mathbf{x}^{*}\right]\right)}\left\|\nabla_{\Gamma_{h}\left[\mathbf{x}^{*}\right]} e_{x}\right\|_{L^{2}\left(\Gamma_{h}\left[\mathbf{x}^{*}\right]\right)} \\
& \quad \leq c\left\|\mathbf{e}_{\mathbf{v}}\right\|_{\mathbf{K}\left(\mathbf{x}^{*}\right)}\left(\left\|\mathbf{e}_{\mathbf{u}}\right\|_{\mathbf{K}\left(\mathbf{x}^{*}\right)}+\left\|\mathbf{e}_{\mathbf{x}}\right\|_{\mathbf{K}\left(\mathbf{x}^{*}\right)}\right) .
\end{aligned}
$$

Altogether, after an absorption, we then have

$$
\left\|\mathbf{e}_{\mathbf{v}}\right\|_{\mathbf{K}\left(\mathbf{x}^{*}\right)}^{2} \leq c\left\|\mathbf{e}_{\mathbf{x}}\right\|_{\mathbf{K}\left(\mathbf{x}^{*}\right)}^{2}+c\left\|\mathbf{e}_{\mathbf{u}}\right\|_{\mathbf{K}\left(\mathbf{x}^{*}\right)}^{2}+c\left\|\mathbf{d}_{\mathbf{v}}\right\|_{\star, \mathbf{x}^{*}}^{2} .
$$

(C) Combination: We use the equation $\dot{\mathbf{e}}_{\mathbf{x}}=\mathbf{e}_{\mathbf{v}}$ and the bound (7.11) to show the bound

$$
\begin{aligned}
\left\|\mathbf{e}_{\mathbf{x}}(t)\right\|_{\mathbf{K}\left(\mathbf{x}^{*}\right)}^{2} & =\int_{0}^{t} \frac{\mathrm{d}}{\mathrm{d} s}\left\|\mathbf{e}_{\mathbf{x}}(s)\right\|_{\mathbf{K}\left(\mathbf{x}^{*}\right)}^{2} \mathrm{~d} s \\
= & \int_{0}^{t}\left(2 \mathbf{e}_{\mathbf{x}}(s)^{T} \mathbf{K}\left(\mathbf{x}^{*}\right) \dot{\mathbf{e}}_{\mathbf{x}}(s)+\mathbf{e}_{\mathbf{x}}(s)^{T} \frac{\mathrm{d}}{\mathrm{d} s}\left(\mathbf{K}\left(\mathbf{x}^{*}\right)\right) \mathbf{e}_{\mathbf{x}}(s)\right) \mathrm{d} s \\
& \leq c \int_{0}^{t}\left\|\mathbf{e}_{\mathbf{v}}(s)\right\|_{\mathbf{K}\left(\mathbf{x}^{*}\right)}^{2} \mathrm{~d} s+c \int_{0}^{t}\left\|\mathbf{e}_{\mathbf{x}}(s)\right\|_{\mathbf{K}\left(\mathbf{x}^{*}\right)}^{2} \mathrm{~d} s,
\end{aligned}
$$

which is substituted into the estimates (7.34) and (7.33), the latter again plugged into (7.34). Then we take the linear combination of the three inequalities, and obtain

$$
\begin{aligned}
& \left\|\mathbf{e}_{\mathbf{x}}(t)\right\|_{\mathbf{K}\left(\mathbf{x}^{*}(t)\right)}^{2}+\left\|\mathbf{e}_{\mathbf{v}}(t)\right\|_{\mathbf{K}\left(\mathbf{x}^{*}(t)\right)}^{2}+\left\|\mathbf{e}_{\mathbf{u}}(t)\right\|_{\mathbf{K}\left(\mathbf{x}^{*}(t)\right)}^{2} \\
& \leq c \int_{0}^{t}\left(\left\|\mathbf{e}_{\mathbf{x}}(s)\right\|_{\mathbf{K}\left(\mathbf{x}^{*}(s)\right)}^{2}+\left\|\mathbf{e}_{\mathbf{v}}(s)\right\|_{\mathbf{K}\left(\mathbf{x}^{*}(s)\right)}^{2}+\left\|\mathbf{e}_{\mathbf{u}}(s)\right\|_{\mathbf{K}\left(\mathbf{x}^{*}(s)\right)}^{2}\right) \mathrm{d} s \\
& \quad+c\left\|\mathbf{e}_{\mathbf{u}}(0)\right\|_{\mathbf{K}\left(\mathbf{x}^{*}(0)\right)}^{2}+c\left\|\mathbf{d}_{\mathbf{v}}(t)\right\|_{\star, \mathbf{x}^{*}(t)}^{2}+c \int_{0}^{t}\left\|\mathbf{d}_{\mathbf{u}}(s)\right\|_{\mathbf{M}\left(\mathbf{x}^{*}(s)\right)}^{2} \mathrm{~d} s .
\end{aligned}
$$

Finally, by Gronwall's inequality we obtain the stability bound (7.18) for $t \in$ $\left[0, t^{*}\right]$.

Now it only remains to show that $t^{*}=T$ for $h$ sufficiently small. To this end we use the assumed defect bounds to obtain the error estimates of order $\kappa$ :

$$
\left\|\mathbf{e}_{\mathbf{v}}(t)\right\|_{\mathbf{K}\left(\mathbf{x}^{*}\right)}+\left\|\mathbf{e}_{\mathbf{u}}(t)\right\|_{\mathbf{K}\left(\mathbf{x}^{*}\right)}+\left\|\mathbf{e}_{\mathbf{x}}(t)\right\|_{\mathbf{K}\left(\mathbf{x}^{*}\right)} \leq C h^{\kappa} .
$$

Then, by the inverse inequality, we have for $t \in\left[0, t^{*}\right]$

$$
\begin{aligned}
& \left\|e_{v}(\cdot, t)\right\|_{W^{1, \infty}\left(\Gamma_{h}\left[\mathbf{x}^{*}(t)\right]\right)}+\left\|e_{u}(\cdot, t)\right\|_{W^{1, \infty}\left(\Gamma_{h}\left[\mathbf{x}^{*}(t)\right]\right)}+\left\|e_{x}(\cdot, t)\right\|_{W^{1, \infty}\left(\Gamma_{h}\left[\mathbf{x}^{*}(t)\right]\right)} \\
& \leq c h^{-1}\left(\left\|\mathbf{e}_{\mathbf{v}}(t)\right\|_{\mathbf{K}\left(\mathbf{x}^{*}\right)}+\left\|\mathbf{e}_{\mathbf{u}}(t)\right\|_{\mathbf{K}\left(\mathbf{x}^{*}\right)}+\left\|\mathbf{e}_{\mathbf{x}}(t)\right\|_{\mathbf{K}\left(\mathbf{x}^{*}\right)}\right) \\
& \leq c C h^{\kappa-1} \leq \frac{1}{2} h^{(\kappa-1) / 2}
\end{aligned}
$$


for sufficiently small $h$. Hence we can extend the bounds (7.21) beyond $t^{*}$, which contradicts the maximality of $t^{*}$ unless $t^{*}=T$. Therefore we have the stability bound (7.18) for $t \in[0, T]$.

Remark 7.1 One might be tempted, as we originally were, to use the above arguments also for proving the stability of Dziuk's method. Unfortunately, this does not work out. Let us explain what difficulties arise.

The error equation for Dziuk's method (3.6) looks like (7.16b)-(7.16c), with $\mathbf{e}_{\mathbf{x}}$ in place of $\mathbf{e}_{\mathbf{u}}$ and $\mathbf{f} \equiv 0$. This then leads to an estimate like (7.30), again with $\mathbf{e}_{\mathbf{x}}$ in place of $\mathbf{e}_{\mathbf{u}}$ and with the last but one line dropped, i.e.,

$$
\begin{aligned}
& \left\|\dot{\mathbf{e}}_{\mathbf{x}}\right\|_{\mathbf{M}(\mathbf{x})}^{2}+\frac{1}{2} \frac{\mathrm{d}}{\mathrm{d} t}\left\|\mathbf{e}_{\mathbf{x}}\right\|_{\mathbf{A}(\mathbf{x})}^{2} \\
& \leq c \\
& \quad+\mathbf{e}_{\mathbf{x}}\left\|_{\mathbf{A}\left(\mathbf{x}^{*}\right)}^{2}+c\right\| \dot{\mathbf{e}}_{\mathbf{x}}\left\|_{\mathbf{M}\left(\mathbf{x}^{*}\right)}\right\| \mathbf{e}_{\mathbf{x}}\left\|_{\mathbf{K}\left(\mathbf{x}^{*}\right)}+c h^{(\kappa-1) / 2}\right\| \dot{\mathbf{e}}_{\mathbf{x}} \|_{\mathbf{M}\left(\mathbf{x}^{*}\right)}^{2} \\
& \quad+c\left\|\mathbf{e}_{\mathbf{x}}\right\|_{\mathbf{A}\left(\mathbf{x}^{*}\right)}\left(\left\|\mathbf{e}_{\mathbf{v}}\right\|_{\mathbf{K}\left(\mathbf{x}^{*}\right)}+\left\|\mathbf{e}_{\mathbf{x}}\right\|_{\mathbf{K}\left(\mathbf{x}^{*}\right)}\right) \\
& \quad-\frac{\mathrm{d}}{\mathrm{d} t}\left(\mathbf{e}_{\mathbf{x}}^{T}\left(\mathbf{A}(\mathbf{x})-\mathbf{A}\left(\mathbf{x}^{*}\right)\right) \mathbf{x}^{*}\right) \\
& \quad+\left\|\dot{\mathbf{e}}_{\mathbf{x}}\right\|_{\mathbf{M}\left(\mathbf{x}^{*}\right)}\left\|\mathbf{d}_{\mathbf{u}}\right\|_{\mathbf{M}\left(\mathbf{x}^{*}\right)}
\end{aligned}
$$

The term $\left\|\mathbf{e}_{\mathbf{v}}\right\|_{\mathbf{K}\left(\mathbf{x}^{*}\right)}$ in the inequality above cannot be bounded by the lefthand side, which contains only $\left\|\dot{\mathbf{e}}_{\mathbf{x}}\right\|_{\mathbf{M}\left(\mathbf{x}^{*}\right)}^{2}$ instead of $\left\|\dot{\mathbf{e}}_{\mathbf{x}}\right\|_{\mathbf{K}\left(\mathbf{x}^{*}\right)}^{2}$ (recall that $\mathbf{e}_{\mathbf{v}}=\dot{\mathbf{e}}_{\mathbf{x}}$ by $\left.(7.16 \mathrm{c})\right)$. By considering the enlarged system, we manage to bound the term $\left\|\mathbf{e}_{\mathbf{v}}\right\|_{\mathbf{K}\left(\mathbf{x}^{*}\right)}$ in (7.30) by using (7.34) derived from testing (7.16a) with $\mathbf{e}_{\mathbf{v}}$. But (7.16a) does not exist in the error equations for Dziuk's method (3.6).

On the other hand, the above arguments work well for the dynamic velocity law $\partial^{\bullet} v=\Delta_{\Gamma[X]} v$ instead of the velocity law $v=\Delta_{\Gamma[X]} x_{\Gamma[X]}$ of mean curvature flow; see [33] and [34] where the evolving finite element semi-discretization and full discretization, respectively, of such a dynamic velocity law were studied. The dynamic velocity law $\partial^{\bullet} v=\Delta_{\Gamma[X]} v$ would yield an error equation similar to (7.16a), which can help to bound the term $\left\|\mathbf{e}_{\mathbf{v}}\right\|_{\mathbf{K}\left(\mathbf{x}^{*}\right)}$. However, taking the time derivative in the velocity law $v=\Delta_{\Gamma[X]} x_{\Gamma[X]}$ of mean curvature flow (or in its weak formulation (2.7) or in its finite element discretization (3.3)) does not lead to a dynamic velocity law with a coercive operator on the right-hand side, but instead the differentiation of (3.3) yields

$$
\frac{\mathrm{d}}{\mathrm{d} t} \int_{\Gamma_{h}} v_{h} \cdot \varphi_{h}+\int_{\Gamma_{h}}\left(\nabla_{\Gamma_{h}} v_{h} \cdot \nabla_{\Gamma_{h}} \varphi_{h}+\nabla_{\Gamma_{h}} x_{h} \cdot\left(D_{\Gamma_{h}} v_{h}\right) \nabla_{\Gamma_{h}} \varphi_{h}\right)=0
$$

for all $\varphi_{h} \in S_{h}[\mathbf{x}]^{3}$, where the operator $D_{\Gamma_{h}}$ is defined in Lemma 7.1 and where we note that $\nabla_{\Gamma_{h}} x_{h}=I-\nu_{\Gamma_{h}} \nu_{\Gamma_{h}}^{T}$. The right-hand integral is not given by a $H^{1}$-elliptic bilinear form, in contrast to its first term: a calculation shows that for $\varphi_{h}=v_{h}$ the last term of the integrand is everywhere non-positive, and the complete integrand in the second integral becomes negative in points where $\nabla_{\Gamma_{h}} v_{h}$ has the normal vector $\nu_{\Gamma_{h}}$ in its null-space and has two non-zero 
eigenvalues of different sign (one eigenvalue is always zero, since the columns of $\nabla_{\Gamma_{h}} v_{h}$ are orthogonal to $\left.\nu_{\Gamma_{h}}\right)$.

So we must concede that our techniques do not yield a stability proof for Dziuk's method. It remains an open problem whether a stability result similar to Proposition 7.1 exists at all for Dziuk's method.

\section{Defect bounds for the semi-discretization}

We now turn to estimating the consistency errors defined by (7.14).

Lemma 8.1 Let the surface $X$ evolving under mean curvature flow be sufficiently regular on the time interval $[0, T]$. Then, there exist $h_{0}>0$ and $c>0$ such that for all $h \leq h_{0}$ and for all $t \in[0, T]$, the defects $d_{v}(t) \in S_{h}\left(\Gamma_{h}\left[\mathbf{x}^{*}\right]\right)^{3}$ and $d_{u}(t) \in S_{h}\left(\Gamma_{h}\left[\mathbf{x}^{*}\right]\right)^{4}$ of the kth-degree finite elements, as defined by their nodal vectors $\mathbf{d}_{\mathbf{v}}(t)$ and $\mathbf{d}_{\mathbf{u}}(t)$ in (7.14), are bounded as

$$
\begin{aligned}
\left\|\mathbf{d}_{\mathbf{v}}(t)\right\|_{*, \mathbf{x}^{*}(t)} & =\left\|d_{v}\right\|_{H_{h}^{-1}\left(\Gamma_{h}\left[\mathbf{x}^{*}(t)\right]\right)} \leq c h^{k}, \\
\left\|\mathbf{d}_{\mathbf{u}}(t)\right\|_{\mathbf{M}\left(\mathbf{x}^{*}(t)\right)} & =\left\|d_{u}\right\|_{L^{2}\left(\Gamma_{h}\left[\mathbf{x}^{*}(t)\right]\right)} \leq c h^{k} .
\end{aligned}
$$

The constant $c$ is independent of $h$ and $t \in[0, T]$.

Proof The proof is very similar to the proof of the defect bounds in [33]. The main difference is that we insert the Ritz projection $u_{h}^{*}$ instead of the nodal interpolation into the scheme. This has the benefit that a critical term in estimating the $L^{2}$ norm cancels, as we will see below. On the other hand, the approximation quality of the Ritz projection is the same as that of interpolation: as is shown in [32, Theorem 6.3], we have

$$
\begin{aligned}
& \left\|\left(u_{h}^{*}\right)^{l}(t)-u(t)\right\|_{H^{1}(\Gamma[X(\cdot, t)])} \| \leq C h^{k}, \\
& \left\|\left(\partial_{h}^{\bullet} u_{h}^{*}\right)^{l}(t)-\partial^{\bullet} u(t)\right\|_{H^{1}(\Gamma[X(\cdot, t)])} \| \leq C h^{k} .
\end{aligned}
$$

We begin by rewriting the equations (7.14) defining the defects in weak form. With the functions of $u=(\nu, H)$ defined by $g(u)=-H \nu$ and $f\left(u, \nabla_{\Gamma} u\right)=$ $|A|^{2} u$ for $A=\nabla_{\Gamma} \nu$ we have

$$
\begin{aligned}
& \int_{\Gamma_{h}\left[\mathbf{x}^{*}\right]} \nabla_{\Gamma_{h}\left[\mathbf{x}^{*}\right]} v_{h}^{*} \cdot \nabla_{\Gamma_{h}\left[\mathbf{x}^{*}\right]} \psi_{h}+\int_{\Gamma_{h}\left[\mathbf{x}^{*}\right]} v_{h}^{*} \cdot \psi_{h} \\
& =\int_{\Gamma_{h}\left[\mathbf{x}^{*}\right]} \nabla_{\Gamma_{h}\left[\mathbf{x}^{*}\right]}\left(g\left(u_{h}^{*}\right)\right) \cdot \nabla_{\Gamma_{h}\left[\mathbf{x}^{*}\right]} \psi_{h}+\int_{\Gamma_{h}\left[\mathbf{x}^{*}\right]} g\left(u_{h}^{*}\right) \cdot \psi_{h} \\
& \quad+\int_{\Gamma_{h}\left[\mathbf{x}^{*}\right]} d_{v} \cdot \psi_{h}
\end{aligned}
$$

for all $\psi_{h} \in S_{h}\left[\mathbf{x}^{*}\right]^{3}$, and

$$
\begin{aligned}
& \int_{\Gamma_{h}\left[\mathbf{x}^{*}\right]} \partial \dot{h} u_{h}^{*} \cdot \varphi_{h}+\int_{\Gamma_{h}\left[\mathbf{x}^{*}\right]} \nabla_{\Gamma_{h}\left[\mathbf{x}^{*}\right]} u_{h}^{*} \cdot \nabla_{\Gamma_{h}\left[\mathbf{x}^{*}\right]} \varphi_{h}=\int_{\Gamma_{h}\left[\mathbf{x}^{*}\right]} f\left(u_{h}^{*}, \nabla_{\Gamma_{h}\left[\mathbf{x}^{*}\right]} u_{h}^{*}\right) \cdot \varphi_{h} \\
& \quad+\int_{\Gamma_{h}\left[\mathbf{x}^{*}\right]} d_{u} \cdot \varphi_{h}
\end{aligned}
$$


for all $\varphi_{h} \in S_{h}\left[\mathbf{x}^{*}\right]^{4}$. Subtracting the weak formulation (2.6) of the equations for the exact solution, we thus have

$$
\begin{aligned}
& \int_{\Gamma_{h}\left[\mathbf{x}^{*}\right]} d_{v} \cdot \psi_{h}=\left(\int_{\Gamma_{h}\left[\mathbf{x}^{*}\right]} \nabla_{\Gamma_{h}\left[\mathbf{x}^{*}\right]} v_{h}^{*} \cdot \nabla_{\Gamma_{h}\left[\mathbf{x}^{*}\right]} \psi_{h}-\int_{\Gamma[X]} \nabla_{\Gamma[X]} v \cdot \nabla_{\Gamma[X]} \psi_{h}^{l}\right) \\
& +\left(\int_{\Gamma_{h}\left[\mathbf{x}^{*}\right]} v_{h}^{*} \cdot \psi_{h}-\int_{\Gamma[X]} v \cdot \psi_{h}^{l}\right) \\
& -\left(\int_{\Gamma_{h}\left[\mathbf{x}^{*}\right]} \nabla_{\Gamma_{h}\left[\mathbf{x}^{*}\right]}\left(g\left(u_{h}^{*}\right)\right) \cdot \nabla_{\Gamma_{h}\left[\mathbf{x}^{*}\right]} \psi_{h}-\int_{\Gamma[X]} \nabla_{\Gamma[X]}(g(u)) \cdot \nabla_{\Gamma[X]} \psi_{h}^{l}\right) \\
& -\left(\int_{\Gamma_{h}\left[\mathbf{x}^{*}\right]} g\left(u_{h}^{*}\right) \cdot \psi_{h}-\int_{\Gamma[X]} g(u) \cdot \psi_{h}^{l}\right)
\end{aligned}
$$

for all $\psi_{h} \in S_{h}\left[\mathbf{x}^{*}\right]^{3}$, and

$$
\begin{aligned}
& \int_{\Gamma_{h}\left[\mathbf{x}^{*}\right]} d_{u} \cdot \varphi_{h}=\left(\int_{\Gamma_{h}\left[\mathbf{x}^{*}\right]} \partial_{h}^{\bullet} u_{h}^{*} \cdot \varphi_{h}-\int_{\Gamma[X]} \partial^{\bullet} u \cdot \varphi_{h}^{l}\right) \\
& +\left[\int_{\Gamma_{h}\left[\mathbf{x}^{*}\right]} \nabla_{\Gamma_{h}\left[\mathbf{x}^{*}\right]} u_{h}^{*} \cdot \nabla_{\Gamma_{h}\left[\mathbf{x}^{*}\right]} \varphi_{h}-\int_{\Gamma[X]} \nabla_{\Gamma[X]} u \cdot \nabla_{\Gamma[X]} \varphi_{h}^{l}\right] \\
& -\left(\int_{\Gamma_{h}\left[\mathbf{x}^{*}\right]} f\left(u_{h}^{*}, \nabla_{\Gamma_{h}\left[\mathbf{x}^{*}\right]} u_{h}^{*}\right) \cdot \varphi_{h}-\int_{\Gamma[X]} f\left(u, \nabla_{\Gamma[X]} u\right) \cdot \varphi_{h}^{l}\right)
\end{aligned}
$$

for all $\varphi_{h} \in S_{h}\left[\mathbf{x}^{*}\right]^{4}$. Here we note that the critical term in big square brackets equals

$$
-\left[\int_{\Gamma_{h}\left[\mathbf{x}^{*}\right]} u_{h}^{*} \cdot \varphi_{h}-\int_{\Gamma[X]} u \cdot \varphi_{h}^{l}\right]
$$

thanks to the definition of $u_{h}^{*}$ in (7.13). The remaining terms do not contain the gradient of the test function. This allows us obtain estimates of $d_{u}$ in the $L^{2}$ norm.

From here on, all the terms in brackets can be bounded by the same arguments as for the corresponding terms in [33], using the bound (8.1) instead of the bound for the interpolation error where required. These arguments are based on geometric estimates that were previously proved in [17, 18, 21, 22,32]. We refer to [33] for the details.

\section{Proof of Theorem 4.1}

The errors are decomposed using interpolations for $X$ and $v$ and the Ritz map (7.13) for $u$ and using the definition of the composed lift $L$ from Section 3.4:

$$
\begin{aligned}
X_{h}^{L}-X & =\left(\widehat{X}_{h}-X_{h}^{*}\right)^{l}+\left(\left(X_{h}^{*}\right)^{l}-X\right), \\
v_{h}^{L}-v & =\left(\widehat{v}_{h}-v_{h}^{*}\right)^{l}+\left(\left(v_{h}^{*}\right)^{l}-v\right), \\
u_{h}^{L}-u & =\left(\widehat{u}_{h}-u_{h}^{*}\right)^{l}+\left(\left(u_{h}^{*}\right)^{l}-u\right) .
\end{aligned}
$$


The last terms in these formulas can be bounded in the $H^{1}(\Gamma)$ norm by $C h^{k}$, using the interpolation error bounds from [17, Proposition 2.7] for $X$ and $v$, and the Ritz-map error bound (8.1) for $u$.

To bound the first terms on the right-hand sides, we use the stability estimate of Proposition 7.1 together with the defect bounds of Lemma 8.1 to obtain

$$
\left\|\mathbf{e}_{\mathbf{x}}\right\|_{\mathbf{K}\left(\mathbf{x}^{*}\right)}+\left\|\mathbf{e}_{\mathbf{v}}\right\|_{\mathbf{K}\left(\mathbf{x}^{*}\right)}+\left\|\mathbf{e}_{\mathbf{u}}\right\|_{\mathbf{K}\left(\mathbf{x}^{*}\right)} \leq C h^{k} .
$$

By the equivalence of norms shown in [18, Lemma 3] and by (7.3) we have (omitting the argument $t$ )

$$
\left\|\left(\widehat{u}_{h}-u_{h}^{*}\right)^{l}\right\|_{H^{1}(\Gamma[X])} \leq c\left\|\widehat{u}_{h}-u_{h}^{*}\right\|_{H^{1}\left(\Gamma_{h}\left[\mathbf{x}^{*}\right]\right)}=c\left\|\mathbf{e}_{\mathbf{u}}\right\|_{\mathbf{K}\left(\mathbf{x}^{*}\right)},
$$

and similarly for $\widehat{v}_{h}-v_{h}^{*}$ and $\widehat{X}_{h}-X_{h}^{*}$. This proves the result.

\section{Stability of the full discretization}

\subsection{Auxiliary results by Dahlquist and Nevanlinna \& Odeh}

We recall two important results that enable us to use energy estimates for BDF methods up to order 5: the first result is from Dahlquist's $G$-stability theory, and the second one from the multiplier technique of Nevanlinna and Odeh.

Lemma 10.1 (Dahlquist [11]) Let $\delta(\zeta)=\sum_{j=0}^{q} \delta_{j} \zeta^{j}$ and $\mu(\zeta)=\sum_{j=0}^{q} \mu_{j} \zeta^{j}$ be polynomials of degree at most $q$ (at least one of them of degree $q$ ) that have no common divisor. Let $\langle\cdot, \cdot\rangle$ denote an inner product on $\mathbb{R}^{N}$. If

$$
\operatorname{Re} \frac{\delta(\zeta)}{\mu(\zeta)}>0 \quad \text { for } \quad|\zeta|<1,
$$

then there exists a symmetric positive definite matrix $G=\left(g_{i j}\right) \in \mathbb{R}^{q \times q}$ such that for all $\mathbf{w}_{0}, \ldots, \mathbf{w}_{q} \in \mathbb{R}^{N}$

$$
\left\langle\sum_{i=0}^{q} \delta_{i} \mathbf{w}_{q-i}, \sum_{i=0}^{q} \mu_{i} \mathbf{w}_{q-i}\right\rangle \geq \sum_{i, j=1}^{q} g_{i j}\left\langle\mathbf{w}_{i}, \mathbf{w}_{j}\right\rangle-\sum_{i, j=1}^{q} g_{i j}\left\langle\mathbf{w}_{i-1}, \mathbf{w}_{j-1}\right\rangle .
$$

In view of the following result, the choice $\mu(\zeta)=1-\eta \zeta$ together with the polynomial $\delta(\zeta)$ of the BDF methods will play an important role later on.

Lemma 10.2 (Nevanlinna \& Odeh [38]) If $q \leq 5$, then there exists $0 \leq$ $\eta<1$ such that for $\delta(\zeta)=\sum_{\ell=1}^{q} \frac{1}{\ell}(1-\zeta)^{\ell}$,

$$
\operatorname{Re} \frac{\delta(\zeta)}{1-\eta \zeta}>0 \quad \text { for } \quad|\zeta|<1 .
$$

The smallest possible values of $\eta$ are found to be $\eta=0,0,0.0836,0.2878,0.8160$ for $q=1, \ldots, 5$, respectively. 
These results have previously been applied in the error analysis of BDF methods for various parabolic problems in $[1,2,34,35,36]$, where they were used when testing the error equation with the error. In contrast to these papers, here these results are used for testing the error equation with the discretized time derivative of the error.

\subsection{Defects and errors}

We choose nodal vectors $\mathbf{x}^{*}(t) \in \mathbb{R}^{3 N}, \mathbf{v}^{*}(t) \in \mathbb{R}^{3 N}$ and $\mathbf{u}^{*}(t) \in \mathbb{R}^{4 N}$ determined by the exact solution $X, v$ and $u=(\nu, H)$ as in Section 7.2, and we abbreviate $\mathbf{x}_{*}^{n}=\mathbf{x}^{*}\left(t_{n}\right), \mathbf{v}_{*}^{n}=\mathbf{v}^{*}\left(t_{n}\right)$, and $\mathbf{u}_{*}^{n}=\mathbf{u}^{*}\left(t_{n}\right)$. When we insert these reference values into the numerical scheme, we obtain defects $\mathbf{d}_{\mathbf{v}}^{n}, \mathbf{d}_{\mathbf{u}}^{n}, \mathbf{d}_{\mathbf{x}}^{n}$ that will be studied in the next section: for $n \geq q$,

$$
\begin{aligned}
\mathbf{K}\left(\widetilde{\mathbf{x}}_{*}^{n}\right) \mathbf{v}_{*}^{n} & =\mathbf{g}\left(\widetilde{\mathbf{x}}_{*}^{n}, \widetilde{\mathbf{u}}_{*}^{n}\right)+\mathbf{M}\left(\widetilde{\mathbf{x}}_{*}^{n}\right) \mathbf{d}_{\mathbf{v}}^{n}, \\
\mathbf{M}\left(\widetilde{\mathbf{x}}_{*}^{n}\right) \dot{\mathbf{u}}_{*}^{n}+\mathbf{A}\left(\widetilde{\mathbf{x}}_{*}^{n}\right) \mathbf{u}_{*}^{n} & =\mathbf{f}\left(\widetilde{\mathbf{x}}_{*}^{n}, \widetilde{\mathbf{u}}_{*}^{n}\right)+\mathbf{M}\left(\widetilde{\mathbf{x}}_{*}^{n}\right) \mathbf{d}_{\mathbf{u}}^{n}, \\
\dot{\mathbf{x}}_{*}^{n} & =\mathbf{v}_{*}^{n}+\mathbf{d}_{\mathbf{x}}^{n},
\end{aligned}
$$

where

$$
\begin{aligned}
\widetilde{\mathbf{x}}_{*}^{n}=\sum_{j=0}^{q-1} \gamma_{j} \mathbf{x}_{*}^{n-1-j}, & \widetilde{\mathbf{u}}_{*}^{n}=\sum_{j=0}^{q-1} \gamma_{j} \mathbf{u}_{*}^{n-1-j}, \\
\dot{\mathbf{x}}_{*}^{n}=\frac{1}{\tau} \sum_{j=0}^{q} \delta_{j} \mathbf{x}_{*}^{n-j}, & \dot{\mathbf{u}}_{*}^{n}=\frac{1}{\tau} \sum_{j=0}^{q} \delta_{j} \mathbf{u}_{*}^{n-j} .
\end{aligned}
$$

The errors of the numerical solution values $\mathbf{x}^{n}, \mathbf{v}^{n}$ and $\mathbf{u}^{n}$ are denoted by

$$
\mathbf{e}_{\mathbf{x}}^{n}=\mathbf{x}^{n}-\mathbf{x}_{*}^{n}, \quad \mathbf{e}_{\mathbf{v}}^{n}=\mathbf{v}^{n}-\mathbf{v}_{*}^{n}, \quad \mathbf{e}_{\mathbf{u}}^{n}=\mathbf{u}^{n}-\mathbf{u}_{*}^{n},
$$

and we abbreviate

$$
\dot{\mathbf{e}}_{\mathbf{x}}^{n}=\frac{1}{\tau} \sum_{j=0}^{q} \delta_{j} \mathbf{e}_{\mathbf{x}}^{n-j}, \quad \dot{\mathbf{e}}_{\mathbf{u}}^{n}=\frac{1}{\tau} \sum_{j=0}^{q} \delta_{j} \mathbf{e}_{\mathbf{u}}^{n-j} .
$$

Subtracting (10.1) from (5.1), we obtain the following error equations:

$$
\begin{aligned}
\mathbf{K}\left(\widetilde{\mathbf{x}}^{n}\right) \mathbf{e}_{\mathbf{v}}^{n} & =\mathbf{r}_{\mathbf{v}}^{n}, \\
\mathbf{M}\left(\widetilde{\mathbf{x}}^{n}\right) \dot{\mathbf{e}}_{\mathbf{u}}^{n}+\mathbf{A}\left(\widetilde{\mathbf{x}}^{n}\right) \mathbf{e}_{\mathbf{u}}^{n} & =\mathbf{r}_{\mathbf{u}}^{n}, \\
\dot{\mathbf{e}}_{\mathbf{x}}^{n} & =\mathbf{e}_{\mathbf{v}}^{n}-\mathbf{d}_{\mathbf{x}}^{n},
\end{aligned}
$$

where

$$
\begin{aligned}
\mathbf{r}_{\mathbf{v}}^{n}= & -\left(\mathbf{K}\left(\widetilde{\mathbf{x}}^{n}\right)-\mathbf{K}\left(\widetilde{\mathbf{x}}_{*}^{n}\right)\right) \mathbf{v}_{*}^{n} \\
& +\left(\mathbf{g}\left(\widetilde{\mathbf{x}}^{n}, \widetilde{\mathbf{u}}^{n}\right)-\mathbf{g}\left(\widetilde{\mathbf{x}}_{*}^{n}, \widetilde{\mathbf{u}}_{*}^{n}\right)\right)-\mathbf{M}\left(\widetilde{\mathbf{x}}_{*}^{n}\right) \mathbf{d}_{\mathbf{v}}^{n} \\
\mathbf{r}_{\mathbf{u}}^{n}= & -\left(\mathbf{M}\left(\widetilde{\mathbf{x}}^{n}\right)-\mathbf{M}\left(\widetilde{\mathbf{x}}_{*}^{n}\right)\right) \dot{\mathbf{u}}_{*}^{n} \\
& -\left(\mathbf{A}\left(\widetilde{\mathbf{x}}^{n}\right)-\mathbf{A}\left(\widetilde{\mathbf{x}}_{*}^{n}\right)\right) \mathbf{u}_{*}^{n} \\
& +\left(\mathbf{f}\left(\widetilde{\mathbf{x}}^{n}, \widetilde{\mathbf{u}}^{n}\right)-\mathbf{f}\left(\widetilde{\mathbf{x}}_{*}^{n}, \widetilde{\mathbf{u}}_{*}^{n}\right)\right)-\mathbf{M}\left(\widetilde{\mathbf{x}}_{*}^{n}\right) \mathbf{d}_{\mathbf{u}}^{n}
\end{aligned}
$$


10.3 Stability estimate

The following stability result is the analogue of Proposition 7.1 for the full discretization.

Proposition 10.1 Consider the linearly implicit BDF time discretization of order $q$ with $2 \leq q \leq 5$. Assume that, for step sizes restricted by $\tau \leq C_{0} h$, there exists $\kappa$ with $1<\kappa \leq k$ such that the defects are bounded by

$$
\left\|\mathbf{d}_{\mathbf{x}}^{n}\right\|_{\mathbf{K}\left(\mathbf{x}_{*}^{n}\right)} \leq c h^{\kappa}, \quad\left\|\mathbf{d}_{\mathbf{v}}^{n}\right\|_{\star, \mathbf{x}_{*}^{n}} \leq c h^{\kappa}, \quad\left\|\mathbf{d}_{\mathbf{u}}^{n}\right\|_{\mathbf{M}\left(\mathbf{x}_{*}^{n}\right)} \leq c h^{\kappa}
$$

for $q \tau \leq n \tau \leq T$, and that also the errors of the starting values are bounded by

$$
\left\|\mathbf{e}_{\mathbf{x}}^{i}\right\|_{\mathbf{K}\left(\mathbf{x}_{*}^{n}\right)} \leq c h^{\kappa}, \quad\left\|\mathbf{e}_{\mathbf{v}}^{i}\right\|_{\star, \mathbf{x}_{*}^{n}} \leq c h^{\kappa}, \quad\left\|\mathbf{e}_{\mathbf{u}}^{i}\right\|_{\mathbf{M}\left(\mathbf{x}_{*}^{n}\right)} \leq c h^{\kappa},
$$

for $i=0, \ldots, q-1$. Then, there exist $h_{0}>0$ and $\tau_{0}>0$ such that the following stability estimate holds for all $h \leq h_{0}, \tau \leq \tau_{0}$, and $n$ with $n \tau \leq T$, satisfying $\tau \leq C_{0} h$ (where $C_{0}>0$ can be chosen arbitrarily),

$$
\begin{aligned}
& \left\|\mathbf{e}_{\mathbf{x}}^{n}\right\|_{\mathbf{K}\left(\mathbf{x}_{*}^{n}\right)}^{2}+\left\|\mathbf{e}_{\mathbf{v}}^{n}\right\|_{\mathbf{K}\left(\mathbf{x}_{*}^{n}\right)}^{2}+\left\|\mathbf{e}_{\mathbf{u}}^{n}\right\|_{\mathbf{K}\left(\mathbf{x}_{*}^{n}\right)}^{2} \\
& \leq C \sum_{i=0}^{q-1}\left(\left\|\mathbf{e}_{\mathbf{x}}^{i}\right\|_{\mathbf{K}\left(\mathbf{x}_{*}^{i}\right)}^{2}+\left\|\mathbf{e}_{\mathbf{v}}^{i}\right\|_{\mathbf{K}\left(\mathbf{x}_{*}^{i}\right)}^{2}+\left\|\mathbf{e}_{\mathbf{u}}^{i}\right\|_{\mathbf{K}\left(\mathbf{x}_{*}^{i}\right)}^{2}\right) \\
& \quad+C \max _{0 \leq j \leq n}\left\|\mathbf{d}_{\mathbf{v}}^{j}\right\|_{\star, \mathbf{x}_{*}^{j}}^{2}+C \tau \sum_{j=q}^{n}\left\|\mathbf{d}_{\mathbf{u}}^{j}\right\|_{\mathbf{M}\left(\mathbf{x}_{*}^{j}\right)}^{2}+C \tau \sum_{j=q}^{n}\left\|\mathbf{d}_{\mathbf{x}}^{j}\right\|_{\mathbf{K}\left(\mathbf{x}_{*}^{j}\right)}^{2},
\end{aligned}
$$

where $C$ is independent of $h, \tau$ and $n$ with $n \tau \leq T$, but depends on the final time $T$.

In Section 11 we will show that under sufficient smoothness assumptions on the solution, the defects satisfy the bounds

$$
\left\|\mathbf{d}_{\mathbf{x}}^{n}\right\|_{\mathbf{K}\left(\mathbf{x}_{*}^{n}\right)} \leq C \tau^{q}, \quad\left\|\mathbf{d}_{\mathbf{v}}^{n}\right\|_{\star, \mathbf{x}_{*}^{n}} \leq C\left(h^{k}+\tau^{q}\right), \quad\left\|\mathbf{d}_{\mathbf{u}}^{n}\right\|_{\mathbf{K}\left(\mathbf{x}_{*}^{n}\right)} \leq C\left(h^{k}+\tau^{q}\right) .
$$

Hence, condition (10.5) is satisfied under the step-size restriction $\tau \leq C_{0} h$ if $q \geq 2$. We note that the error functions $e_{x}^{n}, e_{v}^{n} \in S_{h}\left[\mathbf{x}_{*}^{n}\right]^{3}$ and $e_{u}^{n} \in S_{h}\left[\mathbf{x}_{*}^{n}\right]^{4}$ with nodal vectors $\mathbf{e}_{\mathbf{x}}^{n}, \mathbf{e}_{\mathbf{v}}^{n}$ and $\mathbf{e}_{\mathbf{u}}^{n}$, respectively, are then bounded by

$$
\begin{aligned}
&\left\|e_{x}^{n}\right\|_{H^{1}\left(\Gamma_{h}\left(\mathbf{x}_{*}^{n}\right)\right)} \leq C\left(h^{k}+\tau^{q}\right), \\
&\left\|e_{v}^{n}\right\|_{H^{1}\left(\Gamma_{h}\left(\mathbf{x}_{*}^{n}\right)\right)} \leq C\left(h^{k}+\tau^{q}\right), \quad \text { for } n \tau \leq T, \\
&\left\|e_{u}^{n}\right\|_{H^{1}\left(\Gamma_{h}\left(\mathbf{x}_{*}^{n}\right)\right)} \leq C\left(h^{k}+\tau^{q}\right),
\end{aligned}
$$

provided the starting values are sufficiently accurate.

Proof The organisation of the proof, including the numbering of its parts, will be the same as that of Proposition 7.1. 
Let $t^{*} \in(0, T]$ (which a priori might depend on $\tau$ and $h$ ) be the maximal time such that the following inequalities hold:

$$
\begin{aligned}
&\left\|e_{x}^{n}\right\|_{W^{1, \infty}\left(\Gamma_{h}\left[\mathbf{x}_{*}^{n}\right]\right)} \leq h^{(\kappa-1) / 2}, \\
&\left\|e_{v}^{n}\right\|_{W^{1, \infty}\left(\Gamma_{h}\left[\mathbf{x}_{*}^{n}\right]\right)} \leq h^{(\kappa-1) / 2}, \quad \text { for } \quad n \tau \leq t^{*} . \\
&\left\|e_{u}^{n}\right\|_{W^{1, \infty}\left(\Gamma_{h}\left[\mathbf{x}_{*}^{n}\right]\right)} \leq h^{(\kappa-1) / 2},
\end{aligned}
$$

In the following we assume that $n \tau \leq t^{*}$, so that the above bounds are valid. At the end of the proof we will show that in fact $t^{*}$ coincides with $T$.

(A) Estimates for the surface PDE: In the time-continuous case, we tested the error equation for $\mathbf{e}_{\mathbf{u}}$ with the time derivative $\dot{\mathbf{e}}_{\mathbf{u}}$. Now, in the time-discrete case, we form the difference of equation (10.3b) for $n$ with $\eta$ times this equation for $n-1$, for $\eta \in[0,1)$ of Lemma 10.2, and then we test this difference with the discrete time derivative $\dot{\mathbf{e}}_{\mathbf{u}}^{n}$ defined by (10.2). This yields, for $n \geq q+1$,

$$
\begin{aligned}
& \left(\dot{\mathbf{e}}_{\mathbf{u}}^{n}\right)^{T} \mathbf{M}\left(\widetilde{\mathbf{x}}^{n}\right) \dot{\mathbf{e}}_{\mathbf{u}}^{n}-\eta\left(\dot{\mathbf{e}}_{\mathbf{u}}^{n}\right)^{T} \mathbf{M}\left(\widetilde{\mathbf{x}}^{n-1}\right) \dot{\mathbf{e}}_{\mathbf{u}}^{n-1}+\left(\dot{\mathbf{e}}_{\mathbf{u}}^{n}\right)^{T} \mathbf{A}\left(\widetilde{\mathbf{x}}^{n}\right)\left(\mathbf{e}_{\mathbf{u}}^{n}-\eta \mathbf{e}_{\mathbf{u}}^{n-1}\right) \\
& =-\eta\left(\dot{\mathbf{e}}_{\mathbf{u}}^{n}\right)^{T}\left(\mathbf{A}\left(\widetilde{\mathbf{x}}^{n}\right)-\mathbf{A}\left(\widetilde{\mathbf{x}}^{n-1}\right)\right) \mathbf{e}_{\mathbf{u}}^{n-1}+\left(\dot{\mathbf{e}}_{\mathbf{u}}^{n}\right)^{T}\left(\mathbf{r}_{\mathbf{u}}^{n}-\eta \mathbf{r}_{\mathbf{u}}^{n-1}\right) .
\end{aligned}
$$

To be able to deal with the second term in the first line and the first term in the second line (and further terms later in the proof), we need to estimate $\widetilde{\mathbf{x}}^{n}-\widetilde{\mathbf{x}}^{n-1}$. Let

$$
\widetilde{\mathbf{V}}^{n}=\partial^{\tau} \widetilde{\mathbf{x}}^{n}=\frac{1}{\tau}\left(\widetilde{\mathbf{x}}^{n}-\widetilde{\mathbf{x}}^{n-1}\right)
$$

and denote by $\widetilde{V}_{h}^{n} \in S_{h}\left[\widetilde{\mathbf{x}}^{n}\right]$ the finite element function on $\Gamma_{h}\left[\widetilde{\mathbf{x}}^{n}\right]$ with nodal vector $\tilde{\mathbf{V}}^{n}$. We will show that under condition (10.8),

$$
\left\|\widetilde{V}_{h}^{n}\right\|_{W^{1, \infty}\left(\Gamma_{h}\left[\widetilde{\mathbf{x}}^{n}\right]\right)} \leq K
$$

with a constant $K$ independent of $h$ and $\tau$ and $n$ with $n \tau \leq t^{*}$. This condition will play the same crucial role as (7.9) in the time-continuous case. We proceed by induction and assume that this bound holds up to $n-1$. In the same way as (7.9) led to the norm equivalence (7.12), this induction hypothesis implies that for $m=n-1$,

the norms $\|\cdot\|_{\mathbf{M}\left(\widetilde{\mathbf{x}}^{j}\right)}$ are $h$ - and $\tau$-uniformly equivalent for $q \leq j \leq m$,

and so are the norms $\|\cdot\|_{\mathbf{A}\left(\widetilde{\mathbf{x}}^{j}\right)}$.

Next we note that the finite element function $\widetilde{v}_{h}^{n}$ on $\Gamma_{h}\left[\widetilde{\mathbf{x}}^{n-1}\right]$ with nodal vector

$$
\widetilde{\mathbf{v}}^{n}=\sum_{j=0}^{q-1} \gamma_{j} \mathbf{v}^{n-j-1}=\frac{1}{\tau} \sum_{j=0}^{q} \delta_{j} \widetilde{\mathbf{x}}^{n-j}
$$


satisfies, by the norm equivalence (10.11), by the bounds (10.8), and by the fact that finite element interpolation is a bounded operation on $W^{1, \infty}$,

$$
\begin{aligned}
& \left\|\widetilde{v}_{h}^{n}\right\|_{W^{1, \infty}\left(\Gamma_{h}\left[\widetilde{\mathbf{x}}^{n-1}\right]\right)} \leq \sum_{j=0}^{q-1}\left|\gamma_{j}\right|\left\|v_{h}^{n-j-1}\right\|_{W^{1, \infty}\left(\Gamma_{h}\left[\widetilde{\mathbf{x}}^{n-1}\right]\right)} \\
& \leq \sum_{j=0}^{q-1}\left|\gamma_{j}\right|\left\|v_{h}^{n-j-1}\right\|_{W^{1, \infty}\left(\Gamma_{h}\left[\widetilde{\mathbf{x}}^{n-j-1}\right]\right)} \leq \sum_{j=0}^{q-1}\left|\gamma_{j}\right|\left\|v_{h}^{n-j-1}\right\|_{W^{1, \infty}\left(\Gamma_{h}\left[\mathbf{x}_{*}^{n-j-1}\right]\right)} \\
& \leq \sum_{j=0}^{q-1}\left|\gamma_{j}\right|\left(\left\|v_{h, *}^{n-j-1}\right\|_{W^{1, \infty}\left(\Gamma_{h}\left[\mathbf{x}_{*}^{n-j-1}\right]\right)}+\left\|e_{v}^{n-j-1}\right\|_{W^{1, \infty}\left(\Gamma_{h}\left[\mathbf{x}_{*}^{n-j-1}\right]\right)}\right) \\
& \leq \sum_{j=0}^{q-1}\left|\gamma_{j}\right|\left(c\left\|v\left(\cdot, t_{n-j-1}\right)\right\|_{W^{1, \infty}\left(\Gamma_{h}\left[\mathbf{x}_{*}^{n-j-1}\right]\right)}+\left\|e_{v}^{n-j-1}\right\|_{W^{1, \infty}\left(\Gamma_{h}\left[\mathbf{x}_{*}^{n-j-1}\right]\right)}\right) \leq C .
\end{aligned}
$$

We factorize the generating polynomial $\delta(\zeta)=\sum_{j=0}^{q} \delta_{j} \zeta^{j}=\sum_{\ell=1}^{q} \frac{1}{\ell}(1-\zeta)^{\ell}$ as

$$
\delta(\zeta)=(1-\zeta) \sigma(\zeta)
$$

The polynomial $\sigma(\zeta)=\sum_{j=0}^{q-1} \sigma_{j} \zeta^{j}$ has degree $q-1$ and has no zeros in the closed unit disk (this property expresses the zero-stability of the BDF method, which holds true for $q \leq 6$; see [28, Section III.3]). Therefore, there exists $\varrho<1$ such that

$$
\frac{1}{\sigma(\zeta)}=: \chi(\zeta)=\sum_{j=0}^{\infty} \chi_{j} \zeta^{j} \quad \text { with }\left|\chi_{j}\right| \leq c \varrho^{j}
$$

We rewrite, for $n \geq q$,

$$
\widetilde{\mathbf{v}}^{n}=\sum_{j=0}^{q-1} \sigma_{j} \widetilde{\mathbf{V}}^{n-j}=\sum_{j=q}^{n} \sigma_{n-j} \widetilde{\mathbf{V}}^{j}+\mathbf{s}^{n}
$$

with $\sigma_{j}=0$ for $j \geq q$ and $\mathbf{s}^{n}=\sum_{i=1}^{q-1} \sigma_{n-i} \partial^{\tau} \widetilde{\mathbf{x}}^{i}$, for which we note that $\mathbf{s}^{n}=0$ for $n \geq 2 q-2$. We then obtain

$$
\widetilde{\mathbf{V}}^{n}=\sum_{j=q}^{n} \chi_{n-j}\left(\widetilde{\mathbf{v}}^{j}-\mathbf{s}^{j}\right)
$$

because we have (using the associativity of convolution)

$$
\sum_{j=q}^{n} \chi_{n-j}\left(\widetilde{\mathbf{v}}^{j}-\mathbf{s}^{j}\right)=\sum_{j=q}^{n} \chi_{n-j} \sum_{k=q}^{j} \sigma_{j-k} \widetilde{\mathbf{V}}^{k}=\sum_{k=q}^{n}\left(\sum_{m=0}^{n-k} \chi_{n-k-m} \sigma_{m}\right) \tilde{\mathbf{V}}^{k}=\tilde{\mathbf{V}}^{n}
$$

With the geometric decay of the coefficients $\chi_{j}$ and the bound for $\widetilde{\mathbf{v}}^{j}$ and the assumed accuracy of the starting values, we conclude from (10.14) to the bound

$$
\left\|\widetilde{V}_{h}^{n}\right\|_{W^{1, \infty}\left(\Gamma_{h}\left[\widetilde{\mathbf{x}}^{n-1}\right]\right)} \leq C
$$


by the same arguments as used for bounding $\widetilde{v}_{h}^{n}$ above. Here, $C$ depends only on a $W^{1, \infty}$ bound of the exact solution on the interval $[0, T]$. Since $\widetilde{\mathbf{x}}^{n}=$ $\widetilde{\mathbf{x}}^{n-1}+\tau \widetilde{\mathbf{V}}^{n}$, using this bound for $\widetilde{V}_{h}^{n}$ in Lemma 7.2 yields that the norm equivalence (10.11) extends up to $m=n$ (and not just $n-1$ ), and so we obtain (10.10) with $K=2 C$.

After these preparations, the terms in (10.9) are now estimated separately.

(i) On the left-hand side of (10.9), the first term is

$$
\left(\dot{\mathbf{e}}_{\mathbf{u}}^{n}\right)^{T} \mathbf{M}\left(\widetilde{\mathbf{x}}^{n}\right) \dot{\mathbf{e}}_{\mathbf{u}}^{n}=\left\|\dot{\mathbf{e}}_{\mathbf{u}}^{n}\right\|_{\mathbf{M}\left(\widetilde{\mathbf{x}}^{n}\right)}^{2} .
$$

The second term is bounded by

$$
\begin{aligned}
& \left(\dot{\mathbf{e}}_{\mathbf{u}}^{n}\right)^{T} \mathbf{M}\left(\widetilde{\mathbf{x}}^{n-1}\right) \dot{\mathbf{e}}_{\mathbf{u}}^{n-1} \\
& \leq\left\|\dot{\mathbf{e}}_{\mathbf{u}}^{n}\right\|_{\mathbf{M}\left(\widetilde{\mathbf{x}}^{n-1}\right)}\left\|\dot{\mathbf{e}}_{\mathbf{u}}^{n-1}\right\|_{\mathbf{M}\left(\widetilde{\mathbf{x}}^{n-1}\right)} \\
& \leq \frac{1}{2}\left\|\dot{\mathbf{e}}_{\mathbf{u}}^{n}\right\|_{\mathbf{M}\left(\widetilde{\mathbf{x}}^{n-1}\right)}^{2}+\frac{1}{2}\left\|\dot{\mathbf{e}}_{\mathbf{u}}^{n-1}\right\|_{\mathbf{M}\left(\widetilde{\mathbf{x}}^{n-1}\right)}^{2} \\
& \leq \frac{1}{2}(1+c \tau)\left\|\dot{\mathbf{e}}_{\mathbf{u}}^{n}\right\|_{\mathbf{M}\left(\widetilde{\mathbf{x}}^{n}\right)}^{2}+\frac{1}{2}\left\|\dot{\mathbf{e}}_{\mathbf{u}}^{n-1}\right\|_{\mathbf{M}\left(\widetilde{\mathbf{x}}^{n-1}\right)}^{2},
\end{aligned}
$$

where we used the bound (10.10) in (7.8) to raise the superscript from $n-1$ to $n$ in the first term of the last line. This yields

$$
\begin{aligned}
\left(\dot{\mathbf{e}}_{\mathbf{u}}^{n}\right)^{T} \mathbf{M}\left(\widetilde{\mathbf{x}}^{n}\right) \dot{\mathbf{e}}_{\mathbf{u}}^{n}-\eta\left(\dot{\mathbf{e}}_{\mathbf{u}}^{n}\right)^{T} \mathbf{M}\left(\widetilde{\mathbf{x}}^{n-1}\right) \dot{\mathbf{e}}_{\mathbf{u}}^{n-1} & \\
& \geq\left(1-\frac{1}{2} \eta(1+c \tau)\right)\left\|\dot{\mathbf{e}}_{\mathbf{u}}^{n}\right\|_{\mathbf{M}\left(\widetilde{\mathbf{x}}^{n}\right)}^{2}-\frac{1}{2} \eta\left\|\dot{\mathbf{e}}_{\mathbf{u}}^{n-1}\right\|_{\mathbf{M}\left(\widetilde{\mathbf{x}}^{n-1}\right)}^{2} .
\end{aligned}
$$

(ii) We now bound the critical third term on the left-hand side of (10.9) from below. Thanks to Lemmas 10.1 and 10.2 we have

$$
\begin{aligned}
& \left(\dot{\mathbf{e}}_{\mathbf{u}}^{n}\right)^{T} \mathbf{A}\left(\widetilde{\mathbf{x}}^{n}\right)\left(\mathbf{e}_{\mathbf{u}}^{n}-\eta \mathbf{e}_{\mathbf{u}}^{n-1}\right)=\left(\frac{1}{\tau} \sum_{i=0}^{q} \delta_{i} \mathbf{e}_{\mathbf{u}}^{n-i}\right)^{T} \mathbf{A}\left(\widetilde{\mathbf{x}}^{n}\right)\left(\mathbf{e}_{\mathbf{u}}^{n}-\eta \mathbf{e}_{\mathbf{u}}^{n-1}\right) \\
& \geq \frac{1}{\tau} \sum_{i, j=1}^{q} g_{i j}\left(\mathbf{e}_{\mathbf{u}}^{n-q+i}\right)^{T} \mathbf{A}\left(\widetilde{\mathbf{x}}^{n}\right) \mathbf{e}_{\mathbf{u}}^{n-q+j}-\frac{1}{\tau} \sum_{i, j=1}^{q} g_{i j}\left(\mathbf{e}_{\mathbf{u}}^{n-q+i-1}\right)^{T} \mathbf{A}\left(\widetilde{\mathbf{x}}^{n}\right) \mathbf{e}_{\mathbf{u}}^{n-q+j-1} .
\end{aligned}
$$

The first term on the right-hand side of (10.9) is bounded as follows: recalling that $\widetilde{\mathbf{x}}^{n}-\widetilde{\mathbf{x}}^{n-1}=\tau \widetilde{\mathbf{V}}^{n}$ with the bound (10.10) and using the bound (7.8) and the Young inequality yields

$$
\begin{aligned}
-\left(\dot{\mathbf{e}}_{\mathbf{u}}^{n}\right)^{T}\left(\mathbf{A}\left(\widetilde{\mathbf{x}}^{n}\right)-\mathbf{A}\left(\widetilde{\mathbf{x}}^{n-1}\right)\right) \mathbf{e}_{\mathbf{u}}^{n-1} & \leq c \tau\left\|\dot{\mathbf{e}}_{\mathbf{u}}^{n}\right\|_{\mathbf{A}\left(\widetilde{\mathbf{x}}^{n}\right)}\left\|\mathbf{e}_{\mathbf{u}}^{n-1}\right\|_{\mathbf{A}\left(\widetilde{\mathbf{x}}^{n}\right)} \\
& \leq \frac{1}{2} c \rho \tau^{2}\left\|\dot{\mathbf{e}}_{\mathbf{u}}^{n}\right\|_{\mathbf{A}\left(\widetilde{\mathbf{x}}^{n}\right)}^{2}+\frac{1}{2} c \rho^{-1}\left\|\mathbf{e}_{\mathbf{u}}^{n-1}\right\|_{\mathbf{A}\left(\widetilde{\mathbf{x}}^{n}\right)}^{2},
\end{aligned}
$$

where $\rho>0$ will be chosen small, but independent of $h$ and $\tau$. By an inverse inequality we have

$$
\left\|\dot{\mathbf{e}}_{\mathbf{u}}^{n}\right\|_{\mathbf{A}\left(\widetilde{\mathbf{x}}^{n}\right)} \leq \frac{c}{h}\left\|\dot{\mathbf{e}}_{\mathbf{u}}^{n}\right\|_{\mathbf{M}\left(\widetilde{\mathbf{x}}^{n}\right)},
$$

and under the step size restriction $\tau \leq C_{0} h$, we thus obtain

$$
-\left(\dot{\mathbf{e}}_{\mathbf{u}}^{n}\right)^{T}\left(\mathbf{A}\left(\widetilde{\mathbf{x}}^{n}\right)-\mathbf{A}\left(\widetilde{\mathbf{x}}^{n-1}\right)\right) \mathbf{e}_{\mathbf{u}}^{n-1} \leq c \rho\left\|\dot{\mathbf{e}}_{\mathbf{u}}^{n}\right\|_{\mathbf{M}\left(\widetilde{\mathbf{x}}^{n}\right)}^{2}+c \rho^{-1}\left\|\mathbf{e}_{\mathbf{u}}^{n-1}\right\|_{\mathbf{A}\left(\widetilde{\mathbf{x}}^{n-1}\right)}^{2},
$$


where we used again the bound (10.10) in (7.8) to reduce the superscript from $n$ to $n-1$ in the last term.

The last expression on the right-hand side of (10.9) is $\left(\dot{\mathbf{e}}_{\mathbf{u}}^{n}\right)^{T}\left(\mathbf{r}_{\mathbf{u}}^{n}-\eta \mathbf{r}_{\mathbf{u}}^{n-1}\right)$, where $\mathbf{r}_{\mathbf{u}}^{n}$ is defined in (10.4b). The terms that contain the stiffness matrix $\mathbf{A}$ will require new arguments, whereas the other terms are bounded similarly to the corresponding terms in the time-continuous case.

(iii) Using (10.8) in justifying the bound (7.8) and using the Young inequality, the terms involving the differences of mass matrices are bounded as, using the notation $\mathbf{e}_{\widetilde{\mathbf{x}}}^{n}=\widetilde{\mathbf{x}}^{n}-\widetilde{\mathbf{x}}_{*}^{n}=\sum_{i=0}^{q-1} \gamma_{i} \mathbf{e}_{\mathbf{x}}^{n-1-i}$,

$$
\begin{aligned}
& -\left(\dot{\mathbf{e}}_{\mathbf{u}}^{n}\right)^{T}\left(\mathbf{M}\left(\widetilde{\mathbf{x}}^{n}\right)-\mathbf{M}\left(\widetilde{\mathbf{x}}_{*}^{n}\right)\right) \mathbf{u}_{*}^{n}+\eta\left(\dot{\mathbf{e}}_{\mathbf{u}}^{n}\right)^{T}\left(\mathbf{M}\left(\widetilde{\mathbf{x}}^{n-1}\right)-\mathbf{M}\left(\widetilde{\mathbf{x}}_{*}^{n-1}\right)\right) \mathbf{u}_{*}^{n-1} \\
& \leq c\left\|\dot{\mathbf{e}}_{\mathbf{u}}^{n}\right\|_{\mathbf{M}\left(\widetilde{\mathbf{x}}^{n}\right)}\left\|\mathbf{e}_{\widetilde{\mathbf{x}}}^{n}\right\|_{\mathbf{K}\left(\widetilde{\mathbf{x}}^{n}\right)}+c\left\|\dot{\mathbf{e}}_{\mathbf{u}}^{n}\right\|_{\mathbf{M}\left(\widetilde{\mathbf{x}}^{n-1}\right)}\left\|\mathbf{e}_{\widetilde{\mathbf{x}}}^{n-1}\right\|_{\mathbf{K}\left(\widetilde{\mathbf{x}}^{n-1}\right)} \\
& \leq \rho\left\|\dot{\mathbf{e}}_{\mathbf{u}}^{n}\right\|_{\mathbf{M}\left(\widetilde{\mathbf{x}}^{n}\right)}^{2}+c \rho^{-1}\left\|\mathbf{e}_{\widetilde{\mathbf{x}}}^{n}\right\|_{\mathbf{K}\left(\widetilde{\mathbf{x}}^{n}\right)}^{2}+c \rho^{-1}\left\|\mathbf{e}_{\widetilde{\mathbf{x}}}^{n-1}\right\|_{\mathbf{K}\left(\widetilde{\mathbf{x}}^{n-1}\right)}^{2}
\end{aligned}
$$

with a small $\rho>0$, which will be chosen later independently of $h, \tau$ and $n$ with $n \tau \leq t^{*}$.

(iv) We next bound the two terms in $\left(\dot{\mathbf{e}}_{\mathbf{u}}^{n}\right)^{T}\left(\mathbf{r}_{\mathbf{u}}^{n}-\eta \mathbf{r}_{\mathbf{u}}^{n-1}\right)$ that involve the stiffness matrix $\mathbf{A}$ :

$$
\begin{aligned}
R_{\mathbf{A}}^{n} & =R_{\mathbf{A}}^{1, n}+R_{\mathbf{A}}^{2, n} \\
& =-\left(\dot{\mathbf{e}}_{\mathbf{u}}^{n}\right)^{T}\left(\mathbf{A}\left(\widetilde{\mathbf{x}}^{n}\right)-\mathbf{A}\left(\widetilde{\mathbf{x}}_{*}^{n}\right)\right) \mathbf{u}_{*}^{n}+\eta\left(\dot{\mathbf{e}}_{\mathbf{u}}^{n}\right)^{T}\left(\mathbf{A}\left(\widetilde{\mathbf{x}}^{n-1}\right)-\mathbf{A}\left(\widetilde{\mathbf{x}}_{*}^{n-1}\right)\right) \mathbf{u}_{*}^{n-1} .
\end{aligned}
$$

These terms will be bounded using a discrete analogue of the arguments in part (iv) of the proof of Proposition 7.1. We give the detailed argument for the first term, the second term is then bounded in the same way.

Part (iv) of the proof of Proposition 7.1 starts from the product rule of differentiation, which is not directly available in the time-discrete case. So we need to come up with a substitute. From the definition (10.2) of $\dot{\mathbf{e}}_{\mathbf{u}}^{n}$ and the factorization (10.12) we obtain the relation

$$
\dot{\mathbf{e}}_{\mathbf{u}}^{n}=\frac{1}{\tau} \sum_{j=0}^{q} \delta_{j} \mathbf{e}_{\mathbf{u}}^{n-j}=\sum_{j=0}^{q-1} \sigma_{j} \frac{1}{\tau}\left(\mathbf{e}_{\mathbf{u}}^{n-j}-\mathbf{e}_{\mathbf{u}}^{n-j-1}\right)=\sum_{j=0}^{q-1} \sigma_{j} \partial^{\tau} \mathbf{e}_{\mathbf{u}}^{n-j}
$$


We rewrite the term $R_{\mathbf{A}}^{1, n}$ using (10.19) and a discrete product rule formula, and obtain the following discrete substitute for (7.23):

$$
\begin{aligned}
R_{\mathbf{A}}^{1, n} & =-\sum_{j=0}^{q-1} \sigma_{j}\left(\partial^{\tau} \mathbf{e}_{\mathbf{u}}^{n-j}\right)^{T}\left(\mathbf{A}\left(\widetilde{\mathbf{x}}^{n}\right)-\mathbf{A}\left(\widetilde{\mathbf{x}}_{*}^{n}\right)\right) \mathbf{u}_{*}^{n} \\
& =-\sum_{j=0}^{q-1} \sigma_{j} \partial^{\tau}\left(\left(\mathbf{e}_{\mathbf{u}}^{n-j}\right)^{T}\left(\mathbf{A}\left(\widetilde{\mathbf{x}}^{n}\right)-\mathbf{A}\left(\widetilde{\mathbf{x}}_{*}^{n}\right)\right) \mathbf{u}_{*}^{n}\right) \\
& +\sum_{j=0}^{q-1} \sigma_{j}\left(\mathbf{e}_{\mathbf{u}}^{n-j-1}\right)^{T} \partial^{\tau}\left(\mathbf{A}\left(\widetilde{\mathbf{x}}^{n}\right)-\mathbf{A}\left(\widetilde{\mathbf{x}}_{*}^{n}\right)\right) \mathbf{u}_{*}^{n} \\
& +\sum_{j=0}^{q-1} \sigma_{j}\left(\mathbf{e}_{\mathbf{u}}^{n-j-1}\right)^{T}\left(\mathbf{A}\left(\widetilde{\mathbf{x}}^{n-1}\right)-\mathbf{A}\left(\widetilde{\mathbf{x}}_{*}^{n-1}\right)\right) \partial^{\tau} \mathbf{u}_{*}^{n} .
\end{aligned}
$$

We rewrite the summands of the term that contains $\partial^{\tau}\left(\mathbf{A}\left(\widetilde{\mathbf{x}}^{n}\right)-\mathbf{A}\left(\widetilde{\mathbf{x}}_{*}^{n}\right)\right)$. Let us define the intermediate surfaces, for a fixed $n$,

$\widetilde{\Gamma}_{h}^{\xi}=\Gamma_{h}\left[\xi \widetilde{\mathbf{x}}^{n}+(1-\xi) \widetilde{\mathbf{x}}^{n-1}\right] \quad$ and $\quad \widetilde{\Gamma}_{*, h}^{\xi}=\Gamma_{h}\left[\xi \widetilde{\mathbf{x}}_{*}^{n}+(1-\xi) \widetilde{\mathbf{x}}_{*}^{n-1}\right] \quad$ for $\xi \in[0,1]$, with velocities $V_{\widetilde{\Gamma}_{h}^{\xi}}$ and $V_{\widetilde{\Gamma}_{*, h}^{\xi}}$ that are independent of $\xi$ and correspond to the nodal vectors $\widetilde{\mathbf{V}}^{n}=\partial^{\tau} \widetilde{\mathbf{x}}^{n}$ and $\widetilde{\mathbf{V}}_{*}^{n}=\partial^{\tau} \widetilde{\mathbf{x}}_{*}^{n}$, respectively. We denote by $e_{u}^{\xi}$ and $u_{*}^{\xi}$ the finite element functions with nodal vectors $\mathbf{e}_{\mathbf{u}}$ and $\mathbf{u}^{*}$, respectively. We then have, momentarily dropping the superscripts of $\mathbf{e}_{\mathbf{u}}$ and $\mathbf{u}_{*}$,

$$
\begin{aligned}
& \mathbf{e}_{\mathbf{u}}^{T} \partial^{\tau}\left(\mathbf{A}\left(\widetilde{\mathbf{x}}^{n}\right)-\mathbf{A}\left(\widetilde{\mathbf{x}}_{*}^{n}\right)\right) \mathbf{u}_{*} \\
& =\frac{1}{\tau}\left(\mathbf{e}_{\mathbf{u}}^{T}\left(\mathbf{A}\left(\widetilde{\mathbf{x}}^{n}\right)-\mathbf{A}\left(\widetilde{\mathbf{x}}^{n-1}\right)\right) \mathbf{u}_{*}-\mathbf{e}_{\mathbf{u}}^{T}\left(\mathbf{A}\left(\widetilde{\mathbf{x}}_{*}^{n}\right)-\mathbf{A}\left(\widetilde{\mathbf{x}}_{*}^{n-1}\right)\right) \mathbf{u}_{*}\right) \\
& =\int_{0}^{1} \int_{\widetilde{\Gamma}_{h}^{\xi}} \nabla_{\widetilde{\Gamma}_{h}^{\xi}} e_{u}^{\xi} \cdot\left(D_{\widetilde{\Gamma}_{h}^{\xi}} V_{\widetilde{\Gamma}_{h}^{\xi}}\right) \nabla_{\widetilde{\Gamma}_{h}^{\xi}} u_{*}^{\xi} \mathrm{d} \xi \\
& \quad-\int_{0}^{1} \int_{\widetilde{\Gamma}_{*, h}^{\xi}} \nabla_{\widetilde{\Gamma}_{*, h}^{\xi}} e_{u}^{\xi} \cdot\left(D_{\widetilde{\Gamma}_{*, h}^{\xi}} V_{\widetilde{\Gamma}_{*, h}^{\xi}}\right) \nabla_{\widetilde{\Gamma}_{*, h}^{\xi}} u_{*}^{\xi} \mathrm{d} \xi \\
& =\int_{0}^{1} \frac{\mathrm{d}}{\mathrm{d} \theta} \int_{0}^{1} \int_{\widetilde{\Gamma}_{h}^{\xi, \theta}} \nabla_{\widetilde{\Gamma}_{h}^{\xi, \theta}} e_{u}^{\xi, \theta} \cdot\left(D_{\widetilde{\Gamma}_{h}^{\xi, \theta}} V_{\widetilde{\Gamma}_{h}^{\xi, \theta}}\right) \nabla_{\widetilde{\Gamma}_{h}^{\xi, \theta}} u_{*}^{\xi, \theta} \mathrm{d} \xi \mathrm{d} \theta,
\end{aligned}
$$

where $\widetilde{\Gamma}_{h}^{\xi, \theta}=\Gamma_{h}\left[\widetilde{\mathbf{x}}_{*}^{\xi}+\theta \mathbf{e}_{\widetilde{\mathbf{x}}}^{\xi}\right]$, with $\widetilde{\mathbf{x}}_{*}^{\xi}=\xi \widetilde{\mathbf{x}}_{*}^{n}+(1-\xi) \widetilde{\mathbf{x}}_{*}^{n-1}$ and analogously for $\mathbf{e}_{\widetilde{\mathbf{x}}}^{\xi}$, and where $D_{\widetilde{\Gamma}_{h}^{\xi, \theta}} V_{\widetilde{\Gamma}_{h}^{\xi, \theta}}=\operatorname{tr}\left(G_{h}^{\xi, \theta}\right) I_{3}-\left(G_{h}^{\xi, \theta}+\left(G_{h}^{\xi, \theta}\right)^{T}\right)$ with $G_{h}^{\xi, \theta}=$ $\nabla_{\widetilde{\Gamma}_{h}^{\xi, \theta}} V_{\widetilde{\Gamma}_{h}^{\xi, \theta}} \in \mathbb{R}^{3 \times 3}$. Furthermore, we let $E_{V}^{\xi, \theta}=V_{\widetilde{\Gamma}_{h}^{\xi}}-V_{\widetilde{\Gamma}_{*, h}^{\xi}}$ (which corresponds to the nodal values $\left.\mathbf{E}_{\widetilde{\mathbf{V}}}^{n}=\widetilde{\mathbf{V}}^{n}-\widetilde{\mathbf{V}}_{*}^{n}\right)$ and $V_{\widetilde{\Gamma}_{h}^{\xi, \theta}}=V_{\widetilde{\Gamma}_{*, h}^{\xi}}+\theta E_{V}^{\xi, \theta}$, and we have the relations $0=\partial_{\theta}^{\bullet} e_{u}^{\xi, \theta}=\partial_{\theta}^{\bullet} u_{*}^{\xi, \theta}$ (since both functions are independent of $\theta$ ) 
and $\partial_{\theta}^{\bullet} V_{\widetilde{\Gamma}_{h}^{\xi, \theta}}=\partial_{\theta}^{\bullet}\left(V_{\widetilde{\Gamma}_{* h}^{\xi}}+\theta E_{V}^{\xi, \theta}\right)=E_{V}^{\xi, \theta}$. After computing the derivative with respect to $\theta$, and using the formula (7.26), we obtain

$$
\begin{aligned}
& \mathbf{e}_{\mathbf{u}}^{T} \partial^{\tau}\left(\mathbf{A}\left(\widetilde{\mathbf{x}}^{n}\right)-\mathbf{A}\left(\widetilde{\mathbf{x}}_{*}^{n}\right)\right) \mathbf{u}_{*} \\
& =\int_{0}^{1} \int_{0}^{1} \int_{\widetilde{\Gamma}_{h}^{\xi, \theta}} \nabla_{\widetilde{\Gamma}_{h}^{\xi, \theta}} e_{u}^{\xi, \theta} \cdot \partial_{\theta}^{\bullet}\left(D_{\widetilde{\Gamma}_{h}^{\xi, \theta}} V_{\widetilde{\Gamma}_{h}^{\xi, \theta}}\right) \nabla_{\widetilde{\Gamma}_{h}^{\xi, \theta}} u_{*}^{\xi, \theta} \mathrm{d} \xi \mathrm{d} \theta \\
& +\int_{0}^{1} \int_{0}^{1} \int_{\widetilde{\Gamma}_{h}^{\xi, \theta}} \nabla_{\widetilde{\Gamma}_{h}^{\xi, \theta}} e_{u}^{\xi, \theta} \cdot\left(D_{\widetilde{\Gamma}_{h}^{\xi, \theta}} e_{\widetilde{x}}^{\xi, \theta}\right)\left(D_{\widetilde{\Gamma}_{h}^{\xi, \theta}} V_{\widetilde{\Gamma}_{h}^{\xi, \theta}}\right) \nabla_{\widetilde{\Gamma}_{h}^{\xi, \theta}} u_{*}^{\xi, \theta} \mathrm{d} \xi \mathrm{d} \theta .
\end{aligned}
$$

Using the formula (7.27), and that the nodal values of $E_{V}^{\xi, \theta}$ are $\mathbf{E}_{\tilde{\mathbf{V}}}^{n}=\widetilde{\mathbf{V}}^{n}-$ $\widetilde{\mathbf{V}}_{*}^{n}=\partial^{\tau}\left(\widetilde{\mathbf{x}}^{n}-\widetilde{\mathbf{x}}_{*}^{n}\right)=\partial^{\tau} \mathbf{e}_{\widetilde{\mathbf{x}}}^{n}$, and $\mathbf{e}_{\widetilde{\mathbf{x}}}^{n}=\sum_{j=0}^{q-1} \gamma_{j} \mathbf{e}_{\mathbf{x}}^{n-1-j}$, we obtain from the lemmas and bounds of Section 7.1

$$
\begin{aligned}
& \left(\mathbf{e}_{\mathbf{u}}^{n}\right)^{T} \partial^{\tau}\left(\mathbf{A}\left(\widetilde{\mathbf{x}}^{n}\right)-\mathbf{A}\left(\widetilde{\mathbf{x}}_{*}^{n}\right)\right) \mathbf{u}_{*}^{n} \\
& \left.\leq c\left\|u_{*}^{n}\right\|_{W^{1, \infty}\left(\Gamma_{h}\left[\mathbf{x}_{*}^{n}\right]\right)}\right) \mathbf{e}_{\mathbf{u}}^{n} \|_{\mathbf{A}\left(\widetilde{\mathbf{x}}^{n}\right)}\left(\left\|\partial^{\tau} \mathbf{e}_{\widetilde{\mathbf{x}}}^{n}\right\|_{\mathbf{K}\left(\widetilde{\mathbf{x}}^{n}\right)}+\left\|\mathbf{e}_{\widetilde{\mathbf{x}}}^{n}\right\|_{\mathbf{K}\left(\widetilde{\mathbf{x}}^{n}\right)}+\left\|\mathbf{e}_{\widetilde{\mathbf{x}}}^{n-1}\right\|_{\mathbf{K}\left(\widetilde{\mathbf{x}}^{n-1}\right)}\right) \\
& \leq c\left\|\mathbf{e}_{\mathbf{u}}^{n}\right\|_{\mathbf{A}\left(\widetilde{\mathbf{x}}^{n}\right)}\left(\left\|\partial^{\tau} \mathbf{e}_{\widetilde{\mathbf{x}}}^{n}\right\|_{\mathbf{K}\left(\widetilde{\mathbf{x}}^{n}\right)}+\left\|\mathbf{e}_{\widetilde{\mathbf{x}}}^{n}\right\|_{\mathbf{K}\left(\widetilde{\mathbf{x}}^{n}\right)}+\left\|\mathbf{e}_{\widetilde{\mathbf{x}}}^{n-1}\right\|_{\mathbf{K}\left(\widetilde{\mathbf{x}}^{n-1}\right)}\right) \\
& \leq c\left\|\mathbf{e}_{\mathbf{u}}^{n}\right\|_{\mathbf{A}\left(\widetilde{\mathbf{x}}^{n}\right)}\left(\sum_{j=n-q}^{n-1}\left\|\partial^{\tau} \mathbf{e}_{\mathbf{x}}^{j}\right\|_{\mathbf{K}\left(\widetilde{\mathbf{x}}^{j}\right)}+\sum_{j=n-q-1}^{n-1}\left\|\mathbf{e}_{\mathbf{x}}^{j}\right\|_{\mathbf{K}\left(\widetilde{\mathbf{x}}^{j}\right)}\right) \\
& \leq c\left\|\mathbf{e}_{\mathbf{u}}^{n}\right\|_{\mathbf{A}\left(\widetilde{\mathbf{x}}^{n}\right)}^{2}+c\left(\sum_{j=n-q}^{n-1}\left\|\partial^{\tau} \mathbf{e}_{\mathbf{x}}^{j}\right\|_{\mathbf{K}\left(\widetilde{\mathbf{x}}^{j}\right)}^{2}+\sum_{j=n-q-1}^{n-1}\left\|\mathbf{e}_{\mathbf{x}}^{j}\right\|_{\mathbf{K}\left(\widetilde{\mathbf{x}}^{j}\right)}^{2}\right),
\end{aligned}
$$

where the term $D_{\widetilde{\Gamma}_{h}^{\xi, \theta}} V_{\widetilde{\Gamma}_{h}^{\xi, \theta}}$ is bounded using (10.10) and the $W^{1, \infty}$-boundedness of the exact solution.

The last term in (10.20) is bounded, like the corresponding term in the proof of Proposition 7.1, by

$$
\begin{aligned}
& \sum_{j=0}^{q-1} \sigma_{j}\left(\mathbf{e}_{\mathbf{u}}^{n-j-1}\right)^{T}\left(\mathbf{A}\left(\widetilde{\mathbf{x}}^{n-1}\right)-\mathbf{A}\left(\widetilde{\mathbf{x}}_{*}^{n-1}\right)\right) \partial^{\tau} \mathbf{u}_{*}^{n} \\
& \leq c \sum_{j=0}^{q-1}\left\|\mathbf{e}_{\mathbf{u}}^{n-j-1}\right\|_{\mathbf{A}\left(\widetilde{\mathbf{x}}^{n-1}\right)}\left\|\mathbf{e}_{\widetilde{\mathbf{x}}}^{n-1}\right\|_{\mathbf{K}\left(\widetilde{\mathbf{x}}^{n-1}\right)} \\
& \leq c \sum_{j=n-q}^{n-1}\left\|\mathbf{e}_{\mathbf{u}}^{j}\right\|_{\mathbf{A}\left(\widetilde{\mathbf{x}}^{j}\right)}^{2}+c \sum_{j=n-q}^{n-1}\left\|\mathbf{e}_{\mathbf{x}}^{j}\right\|_{\mathbf{K}\left(\widetilde{\mathbf{x}}^{j}\right)}^{2} .
\end{aligned}
$$

In summary, we have shown that

$$
R_{\mathbf{A}}^{1, n}=-\sum_{j=0}^{q-1} \sigma_{j} \partial^{\tau}\left(\left(\mathbf{e}_{\mathbf{u}}^{n-j}\right)^{T}\left(\mathbf{A}\left(\widetilde{\mathbf{x}}^{n}\right)-\mathbf{A}\left(\widetilde{\mathbf{x}}_{*}^{n}\right)\right) \mathbf{u}_{*}^{n}\right)+r_{\mathbf{A}}^{1, n},
$$


where

$$
r_{\mathbf{A}}^{1, n} \leq c \sum_{j=n-q}^{n}\left\|\mathbf{e}_{\mathbf{u}}^{j}\right\|_{\mathbf{A}\left(\widetilde{\mathbf{x}}^{j}\right)}^{2}+c\left(\sum_{j=n-q}^{n-1}\left\|\partial^{\tau} \mathbf{e}_{\mathbf{x}}^{j}\right\|_{\mathbf{K}\left(\widetilde{\mathbf{x}}^{j}\right)}^{2}+\sum_{j=n-q-1}^{n-1}\left\|\mathbf{e}_{\mathbf{x}}^{j}\right\|_{\mathbf{K}\left(\widetilde{\mathbf{x}}^{j}\right)}^{2}\right) .
$$

In the same way we obtain

$$
R_{\mathbf{A}}^{2, n}=\eta \sum_{j=0}^{q-1} \sigma_{j} \partial^{\tau}\left(\left(\mathbf{e}_{\mathbf{u}}^{n-j}\right)^{T}\left(\mathbf{A}\left(\widetilde{\mathbf{x}}^{n-1}\right)-\mathbf{A}\left(\widetilde{\mathbf{x}}_{*}^{n-1}\right)\right) \mathbf{u}_{*}^{n-1}\right)+r_{\mathbf{A}}^{2, n}
$$

with

$$
r_{\mathbf{A}}^{2, n} \leq \eta c \sum_{j=n-q}^{n}\left\|\mathbf{e}_{\mathbf{u}}^{j}\right\|_{\mathbf{A}\left(\widetilde{\mathbf{x}}^{j}\right)}^{2}+\eta c\left(\sum_{j=n-q-1}^{n-2}\left\|\partial^{\tau} \mathbf{e}_{\mathbf{x}}^{j}\right\|_{\mathbf{K}\left(\widetilde{\mathbf{x}}^{j}\right)}^{2}+\sum_{j=n-q-2}^{n-2}\left\|\mathbf{e}_{\mathbf{x}}^{j}\right\|_{\mathbf{K}\left(\widetilde{\mathbf{x}}^{j}\right)}^{2}\right) .
$$

(v) The terms with the nonlinearity are bounded, with a small $\rho>0$, by

$$
\begin{aligned}
& \left(\dot{\mathbf{e}}_{\mathbf{u}}^{n}\right)^{T}\left(\mathbf{f}\left(\widetilde{\mathbf{x}}^{n}, \widetilde{\mathbf{u}}^{n}\right)-\mathbf{f}\left(\widetilde{\mathbf{x}}_{*}^{n}, \widetilde{\mathbf{u}}_{*}^{n}\right)\right)-\eta\left(\dot{\mathbf{e}}_{\mathbf{u}}^{n}\right)^{T}\left(\mathbf{f}\left(\widetilde{\mathbf{x}}^{n-1}, \widetilde{\mathbf{u}}^{n-1}\right)-\mathbf{f}\left(\widetilde{\mathbf{x}}_{*}^{n-1}, \widetilde{\mathbf{u}}_{*}^{n-1}\right)\right) \\
& \leq c\left\|\dot{\mathbf{e}}_{\mathbf{u}}^{n}\right\|_{\mathbf{M}\left(\widetilde{\mathbf{x}}^{n}\right)}\left(\left\|\mathbf{e}_{\widetilde{\mathbf{u}}}^{n}\right\|_{\mathbf{K}\left(\widetilde{\mathbf{x}}^{n}\right)}+\left\|\mathbf{e}_{\widetilde{\mathbf{x}}}^{n}\right\|_{\mathbf{K}\left(\widetilde{\mathbf{x}}^{n}\right)}\right) \\
& \quad+c \eta\left\|\dot{\mathbf{e}}_{\mathbf{u}}^{n}\right\|_{\mathbf{M}\left(\widetilde{\mathbf{x}}^{n-1}\right)}\left(\left\|\mathbf{e}_{\widetilde{\mathbf{u}}}^{n-1}\right\|_{\mathbf{K}\left(\widetilde{\mathbf{x}}^{n-1}\right)}+\left\|\mathbf{e}_{\widetilde{\mathbf{x}}}^{n-1}\right\|_{\mathbf{K}\left(\widetilde{\mathbf{x}}^{n-1}\right)}\right) \\
& \leq \rho\left\|\dot{\mathbf{e}}_{\mathbf{u}}^{n}\right\|_{\mathbf{M}\left(\widetilde{\mathbf{x}}^{n}\right)}^{2}+c\left\|\mathbf{e}_{\widetilde{\mathbf{u}}}^{n}\right\|_{\mathbf{K}\left(\widetilde{\mathbf{x}}^{n}\right)}^{2}+c\left\|_{\widetilde{\mathbf{u}}}^{n-1}\right\|_{\mathbf{K}\left(\widetilde{\mathbf{x}}^{n-1}\right)}^{2} \\
& \quad+c\left\|\mathbf{e}_{\widetilde{\mathbf{x}}}^{n}\right\|_{\mathbf{K}\left(\widetilde{\mathbf{x}}^{n}\right)}^{2}+c\left\|\mathbf{e}_{\widetilde{\mathbf{x}}}^{n-1}\right\|_{\mathbf{K}\left(\widetilde{\mathbf{x}}^{n-1}\right)}^{2} .
\end{aligned}
$$

(vi) The terms with the defects are bounded, with a small $\rho>0$, by

$$
\begin{aligned}
& -\left(\dot{\mathbf{e}}_{\mathbf{u}}^{n}\right)^{T} \mathbf{M}\left(\widetilde{\mathbf{x}}_{*}^{n}\right) \mathbf{d}_{\mathbf{u}}^{n}+\eta\left(\dot{\mathbf{e}}_{\mathbf{u}}^{n}\right)^{T} \mathbf{M}\left(\widetilde{\mathbf{x}}_{*}^{n-1}\right) \mathbf{d}_{\mathbf{u}}^{n-1} \\
& \leq c\left\|\dot{\mathbf{e}}_{\mathbf{u}}^{n}\right\|_{\mathbf{M}\left(\widetilde{\mathbf{x}}^{n}\right)}\left\|\mathbf{d}_{\mathbf{u}}^{n}\right\|_{\mathbf{M}\left(\widetilde{\mathbf{x}}_{*}^{n}\right)}+c \eta\left\|\dot{\mathbf{e}}_{\mathbf{u}}^{n}\right\|_{\mathbf{M}\left(\widetilde{\mathbf{x}}^{n-1}\right)}\left\|\mathbf{d}_{\mathbf{u}}^{n-1}\right\|_{\mathbf{M}\left(\widetilde{\mathbf{x}}_{*}^{n-1}\right)} \\
& \leq \rho\left\|\dot{\mathbf{e}}_{\mathbf{u}}^{n}\right\|_{\mathbf{M}\left(\widetilde{\mathbf{x}}^{n}\right)}^{2}+c\left\|\mathbf{d}_{\mathbf{u}}^{n}\right\|_{\mathbf{M}\left(\widetilde{\mathbf{x}}_{*}^{n}\right)}^{2}+c\left\|\mathbf{d}_{\mathbf{u}}^{n-1}\right\|_{\mathbf{M}\left(\widetilde{\mathbf{x}}_{*}^{n-1}\right)}^{2},
\end{aligned}
$$

where we used the norm equivalences (7.7) between $\widetilde{\mathbf{x}}^{n}$ and $\widetilde{\mathbf{x}}_{*}^{n}$ (for $n-1$ as well), and $\widetilde{\mathbf{x}}_{*}^{n}$ and $\widetilde{\mathbf{x}}_{*}^{n-1}$, (for the former, using the bounds (10.8)).

Inserting the estimates from (i)-(vi) into (10.9) altogether yields the formidable inequality, for $n \geq q+1$ with $n \tau \leq t^{*}$,

$$
\begin{aligned}
& \left((1-c \tau)-\frac{1}{2} \eta(1+c \tau)-c \rho\right)\left\|\dot{\mathbf{e}}_{\mathbf{u}}^{n}\right\|_{\mathbf{M}\left(\widetilde{\mathbf{x}}^{n}\right)}^{2}-\frac{1}{2} \eta\left\|\dot{\mathbf{e}}_{\mathbf{u}}^{n-1}\right\|_{\mathbf{M}\left(\widetilde{\mathbf{x}}^{n-1}\right)}^{2} \\
& \quad+\frac{1}{\tau} \sum_{i, j=1}^{q} g_{i j}\left(\mathbf{e}_{\mathbf{u}}^{n-q+i}\right)^{T} \mathbf{A}\left(\widetilde{\mathbf{x}}^{n}\right) \mathbf{e}_{\mathbf{u}}^{n-q+j}-\frac{1}{\tau} \sum_{i, j=1}^{q} g_{i j}\left(\mathbf{e}_{\mathbf{u}}^{n-q+i-1}\right)^{T} \mathbf{A}\left(\widetilde{\mathbf{x}}^{n}\right) \mathbf{e}_{\mathbf{u}}^{n-q+j-1} \\
& \leq-\sum_{j=0}^{q-1} \sigma_{j} \partial^{\tau}\left(\left(\mathbf{e}_{\mathbf{u}}^{n-j}\right)^{T}\left(\mathbf{A}\left(\widetilde{\mathbf{x}}^{n}\right)-\mathbf{A}\left(\widetilde{\mathbf{x}}_{*}^{n}\right)\right) \mathbf{u}_{*}^{n}\right) \\
& \quad+\eta \sum_{j=0}^{q-1} \sigma_{j} \partial^{\tau}\left(\left(\mathbf{e}_{\mathbf{u}}^{n-j}\right)^{T}\left(\mathbf{A}\left(\widetilde{\mathbf{x}}^{n-1}\right)-\mathbf{A}\left(\widetilde{\mathbf{x}}_{*}^{n-1}\right)\right) \mathbf{u}_{*}^{n-1}\right)+c \varepsilon_{n}
\end{aligned}
$$


with

$$
\begin{aligned}
\varepsilon_{n}= & \sum_{j=0}^{q}\left\|\mathbf{e}_{\mathbf{u}}^{n-j}\right\|_{\mathbf{A}\left(\widetilde{\mathbf{x}}^{n-j}\right)}^{2}+\sum_{j=0}^{q}\left\|\mathbf{e}_{\mathbf{x}}^{n-1-j}\right\|_{\mathbf{K}\left(\widetilde{\mathbf{x}}^{n-1-j}\right)}^{2}+\sum_{j=0}^{q-1}\left\|\partial^{\tau} \mathbf{e}_{\mathbf{x}}^{n-1-j}\right\|_{\mathbf{K}\left(\widetilde{\mathbf{x}}^{n-1-j}\right)}^{2} \\
& +\left\|\mathbf{d}_{\mathbf{u}}^{n}\right\|_{\mathbf{M}\left(\widetilde{\mathbf{x}}_{*}^{n}\right)}^{2}+\left\|\mathbf{d}_{\mathbf{u}}^{n-1}\right\|_{\mathbf{M}\left(\widetilde{\mathbf{x}}_{*}^{n-1}\right)}^{2} .
\end{aligned}
$$

We now sum up the inequality (10.29) from $n=q+1$ to $\bar{n}$ with $\bar{n} \tau \leq t^{*}$ and multiply with the step size $\tau$. We estimate the arising terms separately.

(a) If both $\tau$ and $\rho$ are so small that $(1-c \tau)-\eta(1+c \tau)-c \rho \geq(1-\eta) / 2$ (recall that $\eta<1$ ), then

$$
\begin{aligned}
& \tau \sum_{n=q+1}^{\bar{n}}\left(\left((1-c \tau)-\frac{1}{2} \eta(1+c \tau)-c \rho\right)\left\|\dot{\mathbf{e}}_{\mathbf{u}}^{n}\right\|_{\mathbf{M}\left(\widetilde{\mathbf{x}}^{n}\right)}^{2}-\frac{1}{2} \eta\left\|\dot{\mathbf{e}}_{\mathbf{u}}^{n-1}\right\|_{\mathbf{M}\left(\widetilde{\mathbf{x}}^{n-1}\right)}^{2}\right) \\
& \geq \frac{1}{2}(1-\eta) \tau \sum_{n=q+1}^{\bar{n}}\left\|\dot{\mathbf{e}}_{\mathbf{u}}^{n}\right\|_{\mathbf{M}\left(\widetilde{\mathbf{x}}^{n}\right)}^{2}-\frac{1}{2} \eta \tau\left\|\dot{\mathbf{e}}_{\mathbf{u}}^{q}\right\|_{\mathbf{M}\left(\widetilde{\mathbf{x}}^{q}\right)}^{2} .
\end{aligned}
$$

(b) For the second line in (10.29) we have, with $\gamma>0$ denoting the smallest eigenvalue of the positive definite matrix $\left(g_{i j}\right)$, and using (10.8) with the bound (7.8),

$$
\begin{aligned}
& \tau \sum_{n=q+1}^{\bar{n}}\left(\frac{1}{\tau} \sum_{i, j=1}^{q} g_{i j}\left(\mathbf{e}_{\mathbf{u}}^{n-q+i}\right)^{T} \mathbf{A}\left(\widetilde{\mathbf{x}}^{n}\right) \mathbf{e}_{\mathbf{u}}^{n-q+j}\right. \\
& \left.-\frac{1}{\tau} \sum_{i, j=1}^{q} g_{i j}\left(\mathbf{e}_{\mathbf{u}}^{n-q+i-1}\right)^{T} \mathbf{A}\left(\widetilde{\mathbf{x}}^{n}\right) \mathbf{e}_{\mathbf{u}}^{n-q+j-1}\right) \\
& =\sum_{i, j=1}^{q} g_{i j}\left(\mathbf{e}_{\mathbf{u}}^{\bar{n}-q+i}\right)^{T} \mathbf{A}\left(\widetilde{\mathbf{x}}^{\bar{n}}\right) \mathbf{e}_{\mathbf{u}}^{\bar{n}-q+j}-\sum_{i, j=1}^{q} g_{i j}\left(\mathbf{e}_{\mathbf{u}}^{i}\right)^{T} \mathbf{A}\left(\widetilde{\mathbf{x}}^{q+1}\right) \mathbf{e}_{\mathbf{u}}^{j} \\
& -\sum_{n=q+1}^{\bar{n}-1} \sum_{i, j=1}^{q} g_{i j}\left(\mathbf{e}_{\mathbf{u}}^{n-q+i}\right)^{T}\left(\mathbf{A}\left(\widetilde{\mathbf{x}}^{n+1}\right)-\mathbf{A}\left(\widetilde{\mathbf{x}}^{n}\right)\right) \mathbf{e}_{\mathbf{u}}^{n-q+j} \\
& \geq \gamma \sum_{j=0}^{q-1}\left\|\mathbf{e}_{\mathbf{u}}^{\bar{n}-j}\right\|_{\mathbf{A}\left(\widetilde{\mathbf{x}}^{\bar{n}}\right)}^{2}-c \sum_{i=1}^{q}\left\|\mathbf{e}_{\mathbf{u}}^{i}\right\|_{\mathbf{A}\left(\widetilde{\mathbf{x}}^{q+1}\right)}^{2}-c \tau \sum_{n=q+1}^{\bar{n}-1}\left\|\mathbf{e}_{\mathbf{u}}^{n}\right\|_{\mathbf{A}\left(\widetilde{\mathbf{x}}^{n}\right)}^{2} \\
& \geq \frac{1}{2} \gamma \sum_{j=0}^{q-1}\left\|\mathbf{e}_{\mathbf{u}}^{\bar{n}-j}\right\|_{\mathbf{A}\left(\widetilde{\mathbf{x}}^{\bar{n}-j}\right)}^{2}-c \sum_{i=1}^{q}\left\|\mathbf{e}_{\mathbf{u}}^{i}\right\|_{\mathbf{A}\left(\widetilde{\mathbf{x}}^{i}\right)}^{2}-c \tau \sum_{n=q+1}^{\bar{n}-1}\left\|\mathbf{e}_{\mathbf{u}}^{n}\right\|_{\mathbf{A}\left(\widetilde{\mathbf{x}}^{n}\right)}^{2} .
\end{aligned}
$$


(c) For the first term on the right-hand side of (10.29) we have, after exchanging the sums, a telescoping sum

$$
\begin{aligned}
& -\tau \sum_{n=q+1}^{\bar{n}} \sum_{j=0}^{q-1} \sigma_{j} \partial^{\tau}\left(\left(\mathbf{e}_{\mathbf{u}}^{n-j}\right)^{T}\left(\mathbf{A}\left(\widetilde{\mathbf{x}}^{n}\right)-\mathbf{A}\left(\widetilde{\mathbf{x}}_{*}^{n}\right)\right) \mathbf{u}_{*}^{n}\right) \\
& =-\sum_{j=0}^{q-1} \sigma_{j}\left(\left(\mathbf{e}_{\mathbf{u}}^{\bar{n}-j}\right)^{T}\left(\mathbf{A}\left(\widetilde{\mathbf{x}}^{\bar{n}}\right)-\mathbf{A}\left(\widetilde{\mathbf{x}}_{*}^{\bar{n}}\right)\right) \mathbf{u}_{*}^{\bar{n}}-\left(\mathbf{e}_{\mathbf{u}}^{q-j}\right)^{T}\left(\mathbf{A}\left(\widetilde{\mathbf{x}}^{q}\right)-\mathbf{A}\left(\widetilde{\mathbf{x}}_{*}^{q}\right)\right) \mathbf{u}_{*}^{q}\right) \\
& \leq c \sum_{j=0}^{q-1}\left(\left\|\mathbf{e}_{\mathbf{u}}^{\bar{n}-j}\right\|_{\mathbf{A}\left(\widetilde{\mathbf{x}}^{\bar{n}}\right)}\left\|\mathbf{e}_{\widetilde{\mathbf{x}}}^{\bar{n}}\right\|_{\mathbf{A}\left(\widetilde{\mathbf{x}}^{\bar{n}}\right)}+\left\|\mathbf{e}_{\mathbf{u}}^{q-j}\right\|_{\mathbf{A}\left(\widetilde{\mathbf{x}}^{q}\right)}\left\|\mathbf{e}_{\widetilde{\mathbf{x}}}^{q}\right\|_{\mathbf{A}\left(\widetilde{\mathbf{x}}^{q}\right)}\right) \\
& \leq c \sum_{j=0}^{q-1}\left\|\mathbf{e}_{\mathbf{u}}^{\bar{n}-j}\right\|_{\mathbf{A}\left(\widetilde{\mathbf{x}}^{\bar{n}}\right)} \sum_{j=0}^{q-1}\left\|\mathbf{e}_{\mathbf{x}}^{\bar{n}-1-j}\right\|_{\mathbf{A}\left(\widetilde{\mathbf{x}}^{\bar{n}-1-j}\right)}+c \sum_{j=0}^{q-1}\left\|\mathbf{e}_{\mathbf{u}}^{q-j}\right\|_{\mathbf{A}\left(\widetilde{\mathbf{x}}^{q}\right)}\left\|\mathbf{e}_{\widetilde{\mathbf{x}}^{q}}^{q}\right\|_{\mathbf{A}\left(\widetilde{\mathbf{x}}^{q}\right)} \\
& \leq \frac{1}{4} \gamma \sum_{j=0}^{q-1}\left\|\mathbf{e}_{\mathbf{u}}^{\bar{n}-j}\right\|_{\mathbf{A}\left(\widetilde{\mathbf{x}}^{\bar{n}-j}\right)}^{2}+c \sum_{j=0}^{q-1}\left\|\mathbf{e}_{\mathbf{x}}^{\bar{n}-1-j}\right\|_{\mathbf{A}\left(\widetilde{\mathbf{x}}^{\bar{n}-1-j}\right)}^{2} \\
& \quad+c \sum_{i=0}^{q-1}\left(\left\|\mathbf{e}_{\mathbf{u}}^{i}\right\|_{\mathbf{A}\left(\mathbf{x}^{i}\right)}^{2}+\left\|\mathbf{e}_{\mathbf{x}}^{i}\right\|_{\mathbf{A}\left(\mathbf{x}^{i}\right)}^{2}\right)+c\left\|_{\mathbf{u}}^{q}\right\|_{\mathbf{A}\left(\widetilde{\mathbf{x}}^{q}\right)}^{2},
\end{aligned}
$$

where for the second to last estimate we used that $\mathbf{e}_{\widetilde{\mathbf{x}}}^{n}=\sum_{j=0}^{q-1} \gamma_{j} \mathbf{e}_{\mathbf{x}}^{n-1-j}$ and the norm equivalence (10.11).

A bound of the same type holds for the second term on the right-hand side.

(d) To bound $\mathbf{e}_{\mathbf{u}}^{q}$, we test (10.3b) for $n=q$ with $\dot{\mathbf{e}}_{\mathbf{u}}^{q}$, multiply with $\tau$, and use that $\delta_{0}>0$ and the techniques presented above in (iii)-(vi) to conclude that

$$
\tau\left\|\dot{\mathbf{e}}_{\mathbf{u}}^{q}\right\|_{\mathbf{M}\left(\widetilde{\mathbf{x}}^{q}\right)}^{2}+\delta_{0}\left\|\mathbf{e}_{\mathbf{u}}^{q}\right\|_{\mathbf{A}\left(\widetilde{\mathbf{x}}^{q}\right)}^{2} \leq c \sum_{i=0}^{q-1}\left(\left\|\mathbf{e}_{\mathbf{u}}^{i}\right\|_{\mathbf{A}\left(\mathbf{x}^{i}\right)}^{2}+\left\|\mathbf{e}_{\mathbf{x}}^{i}\right\|_{\mathbf{K}\left(\mathbf{x}^{i}\right)}^{2}\right)+c \tau\left\|\mathbf{d}_{\mathbf{u}}^{q}\right\|_{\mathbf{M}\left(\widetilde{\mathbf{x}}_{*}^{q}\right)}{ }^{2} .
$$

Combining the estimates of (a)-(d), we thus obtain

$$
\begin{aligned}
& \frac{1}{2}(1-\eta) \tau \sum_{n=q+1}^{\bar{n}}\left\|\dot{\mathbf{e}}_{\mathbf{u}}^{n}\right\|_{\mathbf{M}\left(\widetilde{\mathbf{x}}^{n}\right)}^{2}+\frac{1}{4} \gamma \sum_{j=0}^{q-1}\left\|\mathbf{e}_{\mathbf{u}}^{\bar{n}-j}\right\|_{\mathbf{A}\left(\widetilde{\mathbf{x}}^{\bar{n}}-j\right)}^{2} \\
& \leq c \sum_{j=0}^{q-1}\left\|\mathbf{e}_{\mathbf{x}}^{\bar{n}-1-j}\right\|_{\mathbf{A}\left(\widetilde{\mathbf{x}}^{\bar{n}-1-j}\right)}^{2}+c \tau \sum_{n=q+1}^{\bar{n}} \varepsilon_{n} \\
& \quad+c \sum_{i=0}^{q-1}\left(\left\|\mathbf{e}_{\mathbf{u}}^{i}\right\|_{\mathbf{A}\left(\mathbf{x}^{i}\right)}^{2}+\left\|\mathbf{e}_{\mathbf{x}}^{i}\right\|_{\mathbf{A}\left(\mathbf{x}^{i}\right)}^{2}\right)+c \tau\left\|\mathbf{d}_{\mathbf{u}}^{q}\right\|_{\mathbf{M}\left(\widetilde{\mathbf{x}}_{*}^{q}\right)}{ }^{2} .
\end{aligned}
$$

The term $\tau \sum_{n=q+1}^{\bar{n}} \varepsilon_{n}$, which was defined in (10.30), contains a sum of squared norms of $\partial^{\tau} \mathbf{e}_{\mathbf{x}}^{n}=\left(\mathbf{e}_{\mathbf{x}}^{n}-\mathbf{e}_{\mathbf{x}}^{n-1}\right) / \tau$, for which we show

$$
\tau \sum_{n=q}^{\bar{n}}\left\|\partial^{\tau} \mathbf{e}_{\mathbf{x}}^{n}\right\|_{\mathbf{K}\left(\widetilde{\mathbf{x}}^{n}\right)}^{2} \leq c \tau \sum_{n=q}^{\bar{n}}\left\|\dot{\mathbf{e}}_{\mathbf{x}}^{n}\right\|_{\mathbf{K}\left(\widetilde{\mathbf{x}}^{n}\right)}^{2}+\frac{c}{\tau} \sum_{i=0}^{q-1}\left\|\mathbf{e}_{\mathbf{x}}^{i}\right\|_{\mathbf{K}\left(\mathbf{x}^{i}\right)}^{2} .
$$


To prove this inequality, we start from the equation (cf. (10.19))

$$
\dot{\mathbf{e}}_{\mathbf{x}}^{n}=\frac{1}{\tau} \sum_{j=0}^{q} \delta_{j} \mathbf{e}_{\mathbf{x}}^{n-j}=\sum_{j=0}^{q-1} \sigma_{j} \frac{1}{\tau}\left(\mathbf{e}_{\mathbf{x}}^{n-j}-\mathbf{e}_{\mathbf{x}}^{n-j-1}\right)=\sum_{j=0}^{q-1} \sigma_{j} \partial^{\tau} \mathbf{e}_{\mathbf{x}}^{n-j},
$$

which we rewrite, for $n \geq q$, as

$$
\dot{\mathbf{e}}_{\mathbf{x}}^{n}=\sum_{j=q}^{n} \sigma_{n-j} \partial^{\tau} \mathbf{e}_{\mathbf{x}}^{j}+\mathbf{s}^{n},
$$

with $\sigma_{j}=0$ for $j \geq q$ and $\mathbf{s}^{n}=\sum_{i=1}^{q-1} \sigma_{n-i} \partial^{\tau} \mathbf{e}_{\mathbf{x}}^{i}$, for which we note that $\mathbf{s}^{n}=0$ for $n \geq 2 q-1$. We then obtain, as in (10.14) with (10.13),

$$
\partial^{\tau} \mathbf{e}_{\mathbf{x}}^{n}=\sum_{j=q}^{n} \chi_{n-j}\left(\dot{\mathbf{e}}_{\mathbf{x}}^{j}-\mathbf{s}^{j}\right) .
$$

Since the convolution with the absolutely summable sequence $\left(\chi_{j}\right)$ is a bounded operation in $\ell^{2}$ and we have the norm equivalence (10.11) (with $m=\bar{n}$ ), we obtain

$$
\tau \sum_{n=q}^{\bar{n}}\left\|\partial^{\tau} \mathbf{e}_{\mathbf{x}}^{n}\right\|_{\mathbf{K}\left(\widetilde{\mathbf{x}}^{n}\right)}^{2} \leq c \tau \sum_{n=q}^{\bar{n}}\left\|\dot{\mathbf{e}}_{\mathbf{x}}^{n}\right\|_{\mathbf{K}\left(\widetilde{\mathbf{x}}^{n}\right)}^{2}+c \tau \sum_{n=q}^{2 q-2}\left\|\mathbf{s}^{n}\right\|_{\mathbf{K}\left(\mathbf{x}^{i}\right)}^{2},
$$

where the last term is further bounded by

$$
\tau \sum_{n=q}^{2 q-2}\left\|\mathbf{s}^{n}\right\|_{\mathbf{K}\left(\mathbf{x}^{i}\right)}^{2} \leq c \tau \sum_{i=1}^{q-1}\left\|\partial^{\tau} \mathbf{e}_{\mathbf{x}}^{i}\right\|_{\mathbf{K}\left(\mathbf{x}^{i}\right)}^{2} \leq \frac{c}{\tau} \sum_{i=0}^{q-1}\left\|\mathbf{e}_{\mathbf{x}}^{i}\right\|_{\mathbf{K}\left(\mathbf{x}^{i}\right)}^{2} .
$$

This yields (10.32).

For the right-hand side of (10.32) we note that by (10.3c),

$$
\tau \sum_{n=q}^{\bar{n}}\left\|\dot{\mathbf{e}}_{\mathbf{x}}^{n}\right\|_{\mathbf{K}\left(\widetilde{\mathbf{x}}^{n}\right)}^{2} \leq 2 \tau \sum_{n=q}^{\bar{n}}\left\|\mathbf{e}_{\mathbf{v}}^{n}\right\|_{\mathbf{K}\left(\widetilde{\mathbf{x}}^{n}\right)}^{2}+2 \tau \sum_{n=q}^{\bar{n}}\left\|\mathbf{d}_{\mathbf{x}}^{n}\right\|_{\mathbf{K}\left(\widetilde{\mathbf{x}}_{*}^{n}\right)}{ }^{2} .
$$

Next we show that for $\bar{n} \geq q$ with $\bar{n} \tau \leq T$,

$$
\left\|\mathbf{e}_{\mathbf{u}}^{\bar{n}}\right\|_{\mathbf{M}\left(\mathbf{x}_{*}^{\bar{n}}\right)}^{2} \leq c \tau \sum_{n=q}^{\bar{n}}\left\|\dot{\mathbf{e}}_{\mathbf{u}}^{n}\right\|_{\mathbf{M}\left(\mathbf{x}_{*}^{n}\right)}^{2}+c \sum_{i=0}^{q-1}\left\|\mathbf{e}_{\mathbf{u}}^{i}\right\|_{\mathbf{M}\left(\mathbf{x}_{*}^{i}\right)}^{2} .
$$

The proof is similar to that of (10.32). We rewrite

$$
\dot{\mathbf{e}}_{\mathbf{u}}^{n}=\frac{1}{\tau} \sum_{j=q}^{n} \delta_{n-j} \mathbf{e}_{\mathbf{u}}^{j}+\mathbf{s}^{n} \quad \text { with } \quad \mathbf{s}^{n}=\frac{1}{\tau} \sum_{i=0}^{q-1} \delta_{n-i} \mathbf{e}_{\mathbf{u}}^{i}
$$


and $\delta_{j}=0$ for $j>q$, and we note that $\mathbf{s}^{n}=0$ for $n \geq 2 q$. With the coefficients of the power series

$$
\omega(\zeta)=\sum_{n=0}^{\infty} \omega_{n} \zeta^{n}=\frac{1}{\delta(\zeta)}, \quad \text { for } \quad \delta(\zeta)=\sum_{j=0}^{q} \delta_{j} \zeta^{j}
$$

the same argument as in (10.14) gives us, for $n \geq q$,

$$
\mathbf{e}_{\mathbf{u}}^{n}=\tau \sum_{j=q}^{n} \omega_{n-j}\left(\dot{\mathbf{e}}_{\mathbf{u}}^{j}-\mathbf{s}^{j}\right)
$$

By the zero-stability of the BDF method of order $q \leq 6$ (which states that all zeros of $\delta(\zeta)$ are outside the unit circle with the exception of the simple zero at $\zeta=1)$, the coefficients $\omega_{n}$ are bounded: $\left|\omega_{n}\right| \leq c$ for all $n$. We take the $\mathbf{M}\left(\widetilde{\mathbf{x}}^{n}\right)$ norm on both sides and recall that by (10.11) all these norms are uniformly equivalent for $0 \leq n \tau \leq t^{*}$. With the Cauchy-Schwarz inequality we then obtain (10.34).

Recalling the definition (10.30) of $\varepsilon_{n}$ and combining the bounds (10.31)(10.34), we finally obtain (writing here $n$ instead of $\bar{n}$ ) for $n \geq q$ with $n \tau \leq t^{*}$

$$
\begin{aligned}
\left\|\mathbf{e}_{\mathbf{u}}^{n}\right\|_{\mathbf{K}\left(\widetilde{\mathbf{x}}^{n}\right)}^{2} \leq & c \sum_{i=0}^{q-1}\left\|\mathbf{e}_{\mathbf{x}}^{n-1-j}\right\|_{\mathbf{K}\left(\widetilde{\mathbf{x}}^{n-1-j}\right)}^{2} \\
& +c \tau \sum_{j=q}^{n}\left(\left\|\mathbf{e}_{\mathbf{x}}^{j}\right\|_{\mathbf{K}\left(\widetilde{\mathbf{x}}^{j}\right)}^{2}+\left\|\mathbf{e}_{\mathbf{v}}^{j}\right\|_{\mathbf{K}\left(\widetilde{\mathbf{x}}^{j}\right)}^{2}+\left\|\mathbf{e}_{\mathbf{u}}^{j}\right\|_{\mathbf{K}\left(\widetilde{\mathbf{x}}^{j}\right)}^{2}\right) \\
& +c \sum_{i=0}^{q-1}\left(\left\|\mathbf{e}_{\mathbf{u}}^{i}\right\|_{\mathbf{K}\left(\mathbf{x}^{i}\right)}^{2}+\left\|\mathbf{e}_{\mathbf{x}}^{i}\right\|_{\mathbf{K}\left(\mathbf{x}^{i}\right)}^{2}\right) \\
& +c \tau \sum_{j=q}^{n}\left(\left\|\mathbf{d}_{\mathbf{x}}^{j}\right\|_{\mathbf{K}\left(\widetilde{\mathbf{x}}_{*}^{j}\right)}{ }^{2}+\left\|\mathbf{d}_{\mathbf{u}}^{j}\right\|_{\mathbf{M}\left(\widetilde{\mathbf{x}}_{*}^{j}\right)}{ }^{2}\right)
\end{aligned}
$$

From (10.3c) we obtain by the same argument as in the proof of (10.34) a bound for $\mathbf{e}_{\mathbf{x}}^{n}$,

$$
\left\|\mathbf{e}_{\mathbf{x}}^{n}\right\|_{\mathbf{K}\left(\widetilde{\mathbf{x}}^{n}\right)}^{2} \leq c \tau \sum_{j=q}^{n}\left\|\mathbf{e}_{\mathbf{v}}^{j}\right\|_{\mathbf{K}\left(\widetilde{\mathbf{x}}^{j}\right)}^{2}+c \sum_{i=0}^{q-1}\left\|\mathbf{e}_{\mathbf{x}}^{i}\right\|_{\mathbf{K}\left(\mathbf{x}^{i}\right)}^{2}+c \tau \sum_{j=q}^{n}\left\|\mathbf{d}_{\mathbf{x}}^{j}\right\|_{\mathbf{K}\left(\widetilde{\mathbf{x}}_{*}^{j}\right)}{ }^{2} .
$$

(B) Estimates for the velocity equation: We test (10.3a) with $\mathbf{e}_{\mathbf{v}}^{n}$, which yields

$$
\left\|\mathbf{e}_{\mathbf{v}}^{n}\right\|_{\mathbf{K}\left(\widetilde{\mathbf{x}}^{n}\right)}^{2}=\left(\mathbf{e}_{\mathbf{v}}^{n}\right)^{T} \mathbf{K}\left(\widetilde{\mathbf{x}}_{*}^{n}\right) \mathbf{e}_{\mathbf{v}}^{n}=\left(\mathbf{e}_{\mathbf{v}}^{n}\right)^{T} \mathbf{r}_{\mathbf{v}}^{n}
$$

where the right-hand side is estimated in the same way as in part (B) of the proof of Proposition 7.1 to obtain a bound like (7.34) for the discrete error in $v$ :

$$
\left\|\mathbf{e}_{\mathbf{v}}^{n}\right\|_{\mathbf{K}\left(\widetilde{\mathbf{x}}^{n}\right)}^{2} \leq c\left\|\mathbf{e}_{\widetilde{\mathbf{x}}}^{n}\right\|_{\mathbf{K}\left(\widetilde{\mathbf{x}}^{n}\right)}^{2}+c\left\|\mathbf{e}_{\widetilde{\mathbf{u}}}^{n}\right\|_{\mathbf{K}\left(\widetilde{\mathbf{x}}^{n}\right)}^{2}+c\left\|\mathbf{d}_{\mathbf{v}}^{n}\right\|_{\star, \widetilde{\mathbf{x}}_{*}^{n}}^{2},
$$


where $\mathbf{e}_{\widetilde{\mathbf{x}}}^{n}=\widetilde{\mathbf{x}}^{n}-\widetilde{\mathbf{x}}_{*}^{n}=\sum_{i=0}^{q-1} \gamma_{i} \mathbf{e}_{\mathbf{x}}^{n-1-i}$ and $\mathbf{e}_{\widetilde{\mathbf{u}}}^{n}=\widetilde{\mathbf{u}}^{n}-\widetilde{\mathbf{u}}_{*}^{n}=\sum_{i=0}^{q-1} \gamma_{i} \mathbf{e}_{\mathbf{u}}^{n-1-i}$. This yields

$$
\left\|\mathbf{e}_{\mathbf{v}}^{n}\right\|_{\mathbf{K}\left(\widetilde{\mathbf{x}}^{n}\right)}^{2} \leq c \sum_{i=0}^{q-1}\left(\left\|\mathbf{e}_{\mathbf{x}}^{n-1-i}\right\|_{\mathbf{K}\left(\widetilde{\mathbf{x}}^{n-1-i}\right)}^{2}+\left\|\mathbf{e}_{\mathbf{u}}^{n-1-i}\right\|_{\mathbf{K}\left(\widetilde{\mathbf{x}}^{n-1-i}\right)}^{2}\right)+c\left\|\mathbf{d}_{\mathbf{v}}^{n}\right\|_{\star, \widetilde{\mathbf{x}}_{*}^{n}}^{2} .
$$

(C) Combination: Combining the bounds (10.35)-(10.37) and using a discrete Gronwall inequality finally yields the stability estimate (10.7) in the norms that correspond to $\widetilde{\mathbf{x}}^{n}$ instead of $\mathbf{x}_{*}^{n}$. However, these norms are equivalent uniformly in $h, \tau$ and $n$ with $n \tau \leq t^{*}$ by (7.7), since the required smallness condition in the $W^{1, \infty}$ norm is satisfied because of the obtained error bound for $e_{x}$ in the $H^{1}$-norm and an inverse inequality, noting that

$$
\widetilde{\mathbf{x}}^{n}-\mathbf{x}_{*}^{n}=\sum_{j=0}^{q-1} \gamma_{j} \mathbf{e}_{\mathbf{x}}^{n-1-j}+\left(\widetilde{\mathbf{x}}_{*}^{n}-\mathbf{x}_{*}^{n}\right),
$$

where the last term is bounded by $c \tau^{q}$ in the $L^{\infty}$ norm.

Finally, similarly as before, we also obtain $t^{*}=T$, since our error estimates show that the required bounds (10.8) remain satisfied from one step to the next provided that $\tau$ and $h$ are sufficiently small and are related by $\tau \leq C_{0} h$.

\section{Defect bounds for the full discretization}

The consistency errors defined by (10.1) are bounded as follows.

Lemma 11.1 Let the surface $X$ evolving under mean curvature flow be sufficiently regular on the time interval $[0, T]$. Then, there exist $h_{0}>0$ and $\tau_{0}>0$ such that for all $h \leq h_{0}, \tau \leq \tau_{0}$ and $n \geq$ with $n \tau \leq T$, the defects $d_{x}^{n} \in S_{h}\left(\Gamma_{h}\left[\mathbf{x}^{*}\right]\right)^{3}, d_{v}^{n} \in S_{h}\left(\Gamma_{h}\left[\mathbf{x}^{*}\right]\right)^{3}$ and $d_{u}^{n} \in S_{h}\left(\Gamma_{h}\left[\mathbf{x}^{*}\right]\right)^{4}$ of the kth-degree finite elements and the q-step backward difference formula, as defined by their nodal vectors $\mathbf{d}_{\mathbf{x}}^{n}, \mathbf{d}_{\mathbf{v}}^{n}$ and $\mathbf{d}_{\mathbf{u}}^{n}$ in (10.1), are bounded as

$$
\begin{aligned}
\left\|\mathbf{d}_{\mathbf{x}}^{n}\right\|_{\mathbf{K}\left(\mathbf{x}_{*}^{n}\right)} & =\left\|d_{x}^{n}\right\|_{H^{1}\left(\Gamma_{h}\left[\mathbf{x}^{*}\left(t_{n}\right)\right]\right]} \leq c \tau^{q}, \\
\left\|\mathbf{d}_{\mathbf{v}}^{n}\right\|_{\star, \mathbf{x}_{*}^{n}} & =\left\|d_{v}^{n}\right\|_{H_{h}^{-1}\left(\Gamma_{h}\left[\mathbf{x}^{*}\left(t_{n}\right)\right]\right)} \leq c\left(h^{k}+\tau^{q}\right), \\
\left\|\mathbf{d}_{\mathbf{u}}^{n}\right\|_{\mathbf{M}\left(\mathbf{x}_{*}^{n}\right)} & =\left\|d_{u}^{n}\right\|_{L^{2}\left(\Gamma_{h}\left[\mathbf{x}^{*}\left(t_{n}\right)\right]\right]} \leq c\left(h^{k}+\tau^{q}\right) .
\end{aligned}
$$

The constant $c$ is independent of $h, \tau$ and $n$ with $n \tau \leq T$.

Proof Since we have

$$
\mathbf{d}_{\mathbf{x}}^{n}=\frac{1}{\tau} \sum_{j=0}^{q} \delta_{j} \mathbf{x}^{*}\left(t_{n-j}\right)-\dot{\mathbf{x}}^{*}\left(t_{n}\right),
$$

the bound $\left\|\mathbf{d}_{\mathbf{x}}^{n}\right\|_{\mathbf{K}\left(\mathbf{x}_{*}^{n}\right)} \leq c \tau^{p}$ just follows by Taylor expansion. 
Comparing the defects $\mathbf{d}_{\mathbf{v}}\left(t_{n}\right)$ of the semi-discretization, see (7.14a), and $\mathbf{d}_{\mathbf{v}}^{n}$ of the full discretization, see (10.1), we find that they are related by

$\mathbf{M}\left(\widetilde{\mathbf{x}}_{*}^{n}\right) \mathbf{d}_{\mathbf{v}}^{n}=\mathbf{M}\left(\mathbf{x}_{*}^{n}\right) \mathbf{d}_{\mathbf{v}}\left(t_{n}\right)+\left(\mathbf{K}\left(\widetilde{\mathbf{x}}_{*}^{n}\right)-\mathbf{K}\left(\mathbf{x}_{*}^{n}\right)\right) \mathbf{v}_{*}^{n}-\left(\mathbf{g}\left(\widetilde{\mathbf{x}}_{*}^{n}, \widetilde{\mathbf{u}}_{*}^{n}\right)-\mathbf{g}\left(\mathbf{x}_{*}^{n}, \mathbf{u}_{*}^{n}\right)\right)$.

Using Lemma 8.1 to bound $\mathbf{d}_{\mathbf{v}}\left(t_{n}\right)$ and the same arguments as in the proof of Lemma 6.2 in [34] to bound the remaining terms, and $\left\|\widetilde{\mathbf{x}}_{*}^{n}-\mathbf{x}_{*}^{n}\right\|_{\mathbf{K}\left(\mathbf{x}_{*}^{n}\right)} \leq c \tau^{p}$ (see [34, Lemma 6.2]), we obtain the stated bound for $\mathbf{d}_{\mathbf{v}}^{n}$.

Similarly, the defects $\mathbf{d}_{\mathbf{u}}\left(t_{n}\right)$ of the semi-discretization, see $(7.14 \mathrm{~b})$, and $\mathbf{d}_{\mathbf{u}}^{n}$ of the full discretization, see (10.1), are related by

$$
\begin{aligned}
\mathbf{M}\left(\widetilde{\mathbf{x}}_{*}^{n}\right) \mathbf{d}_{\mathbf{u}}^{n}= & \mathbf{M}\left(\mathbf{x}_{*}^{n}\right) \mathbf{d}_{\mathbf{u}}\left(t_{n}\right) \\
& +\mathbf{M}\left(\widetilde{\mathbf{x}}_{*}^{n}\right)\left(\dot{\mathbf{u}}_{*}^{n}-\dot{\mathbf{u}}_{*}\left(t_{n}\right)\right)+\left(\mathbf{M}\left(\widetilde{\mathbf{x}}_{*}^{n}\right)-\mathbf{M}\left(\mathbf{x}_{*}^{n}\right)\right) \dot{\mathbf{u}}_{*}\left(t_{n}\right) \\
& +\left(\mathbf{A}\left(\widetilde{\mathbf{x}}_{*}^{n}\right)-\mathbf{A}\left(\mathbf{x}_{*}^{n}\right)\right) \mathbf{u}_{*}^{n}-\left(\mathbf{f}\left(\widetilde{\mathbf{x}}_{*}^{n}, \widetilde{\mathbf{u}}_{*}^{n}\right)-\mathbf{f}\left(\mathbf{x}_{*}^{n}, \mathbf{u}_{*}^{n}\right)\right) .
\end{aligned}
$$

The term $\mathbf{d}_{\mathbf{u}}\left(t_{n}\right)$ is estimated in Lemma 8.1, while the difference of the discrete and continuous time derivatives is estimated via a Peano kernel representation, see [27, Section 3.2.6], and $\left\|\widetilde{\mathbf{x}}_{*}^{n}-\mathbf{x}_{*}^{n}\right\|_{\mathbf{K}\left(\mathbf{x}^{n}\right)} \leq c \tau^{p}$, from [34, Lemma 6.2]. The further terms are estimated like the analogous terms in the equation for $\mathbf{d}_{\mathbf{v}}^{n}$ above. This yields the stated bounds.

\section{Proof of Theorem 6.1}

With the stability estimate of Proposition 10.1 and the defect bounds of Lemma 11.1 at hand, the proof is now essentially the same as in Section 9. The errors are decomposed using finite element interpolations of $X$ and $v$ and the Ritz map (7.13) for $u$ and using the composed lift $L$ from Section 3.4:

$$
\begin{aligned}
\left(X_{h}^{n}\right)^{L}-X\left(\cdot, t_{n}\right) & =\left(\widehat{X}_{h}^{n}-X_{h}^{*}\left(\cdot, t_{n}\right)\right)^{l}+\left(\left(X_{h}^{*}\left(\cdot, t_{n}\right)\right)^{l}-X\left(\cdot, t_{n}\right)\right), \\
\left(v_{h}^{n}\right)^{L}-v\left(\cdot, t_{n}\right) & =\left(\widehat{v}_{h}^{n}-v_{h}^{*}\left(\cdot, t_{n}\right)\right)^{l}+\left(\left(v_{h}^{*}\left(\cdot, t_{n}\right)\right)^{l}-v\left(\cdot, t_{n}\right)\right), \\
\left(u_{h}^{n}\right)^{L}-u\left(\cdot, t_{n}\right) & =\left(\widehat{u}_{h}^{n}-u_{h}^{*}\left(\cdot, t_{n}\right)\right)^{l}+\left(\left(u_{h}^{*}\left(\cdot, t_{n}\right)\right)^{l}-u\left(\cdot, t_{n}\right)\right) .
\end{aligned}
$$

The last terms in these formulas can be bounded in the $H^{1}(\Gamma)$ norm by $C h^{k}$, using the interpolation and Ritz map error bounds of [32].

To bound the first terms on the right-hand sides, we use the stability estimate of Proposition 10.1 together with the defect bounds of Lemma 11.1 to obtain, for $t_{n}=n \tau \leq T$ and under the required step-size restriction,

$$
\left\|\mathbf{e}_{\mathbf{x}}^{n}\right\|_{\mathbf{K}\left(\mathbf{x}^{*}\left(t_{n}\right)\right)}+\left\|\mathbf{e}_{\mathbf{v}}^{n}\right\|_{\mathbf{K}\left(\mathbf{x}^{*}\left(t_{n}\right)\right)}+\left\|\mathbf{e}_{\mathbf{u}}^{n}\right\|_{\mathbf{K}\left(\mathbf{x}^{*}\left(t_{n}\right)\right)} \leq C\left(h^{k}+\tau^{q}\right) .
$$

By the equivalence of norms shown in [18, Lemma 3] and by (7.3) we have

$$
\begin{aligned}
& \left\|\left(\widehat{u}_{h}^{n}-u_{h}^{*}\left(t_{n}\right)\right)^{l}\right\|_{H^{1}\left(\Gamma\left[X\left(t_{n}\right)\right]\right)} \leq c\left\|\widehat{u}_{h}^{n}-u_{h}^{*}\left(t_{n}\right)\right\|_{H^{1}\left(\Gamma\left[X_{h}^{*}\left(t_{n}\right)\right]\right)} \\
& =c\left\|\widehat{u}_{h}^{n}-u_{h}^{*}\left(t_{n}\right)\right\|_{H^{1}\left(\Gamma_{h}\left[\mathbf{x}^{*}\left(t_{n}\right)\right]\right)}=c\left\|\mathbf{e}_{\mathbf{u}}^{n}\right\|_{\mathbf{K}\left(\mathbf{x}^{*}\left(t_{n}\right)\right)},
\end{aligned}
$$

and similarly for $\widehat{v}_{h}^{n}-v_{h}^{*}\left(t_{n}\right)$ and $\widehat{X}_{h}^{n}-X_{h}^{*}\left(t_{n}\right)$. This proves the result. 

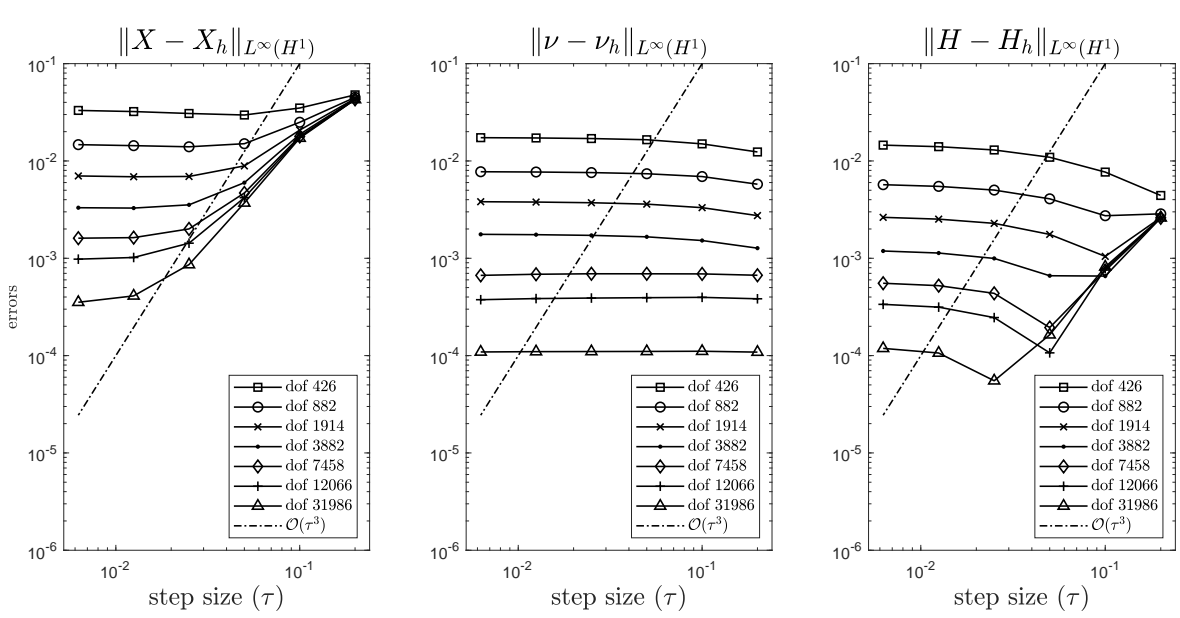

Fig. 13.1 Temporal convergence of the BDF3 / quadratic ESFEM discretisation for MCF for a sphere

\section{Numerical experiments}

We performed the following numerical experiments for mean curvature flow:

- for the sphere, where the exact evolution is known; see, e.g. [30];

- for a dumbbell-shaped surface, where the evolution is known to develop a pinch singularity in finite time; see, e.g. [19]. Here we compare Dziuk's algorithm with the algorithm proposed in this paper and with a variant where the computed normal vector is rescaled to unit norm.

The initial meshes for both examples were generated using DistMesh [39] without taking advantage of the symmetry of the surface, as opposed to [19, Figure 2] and [26, Figure 14].

\subsection{Mean curvature flow for the sphere}

The radius $R(t)$ of a two-dimensional sphere $\Gamma(t)$ evolving according to mean curvature flow satisfies the differential equation, cf. [37]:

$$
\begin{aligned}
R^{\prime}(t) & =-\frac{2}{R(t)}, \quad \text { for } \quad t \in\left[0, T_{\max }\right] \\
R(0) & =R_{0},
\end{aligned}
$$

with solution $R(t)=\sqrt{R_{0}^{2}-4 t}$, which is zero at time $T_{\max }=R_{0}^{2} / 4$. The mean curvature of $\Gamma(t)$ is $H(x, t)=2 / R(t)$, while the normal vector at $x \in \Gamma(t)$ is $\nu(x)=x /|x|$. The surface shrinks to a point at time $T_{\max }$.

Using the algorithm in (5.1) (combining the 3-step BDF method and quadratic evolving surface finite elements) we computed approximations to mean curvature flow for the sphere of radius $R_{0}=2$, over the time interval 

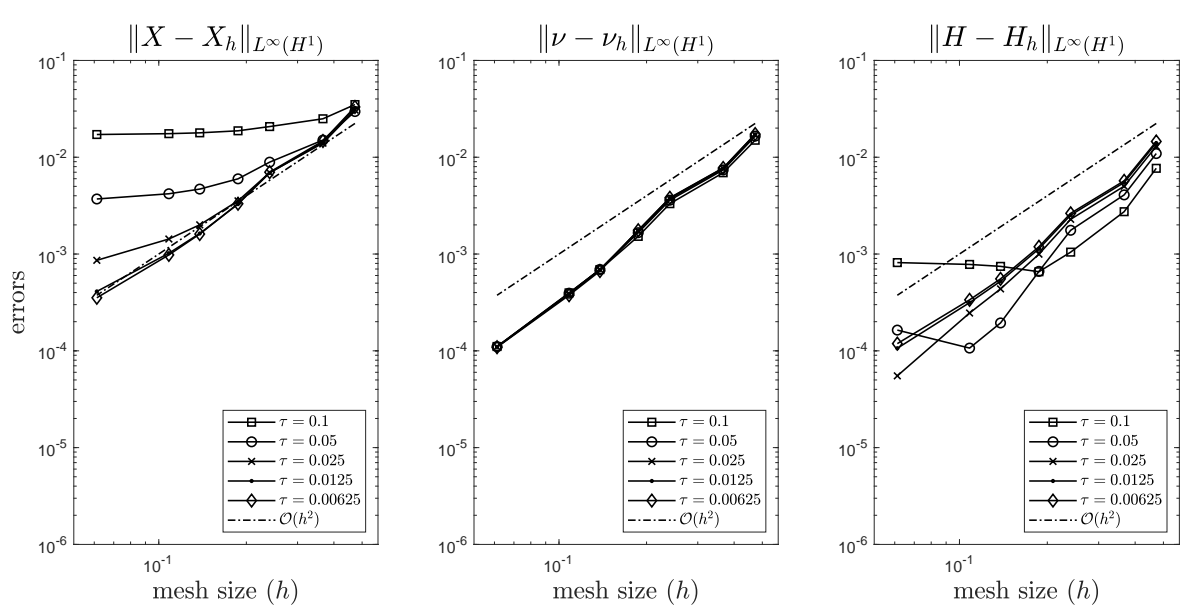

Fig. 13.2 Spatial convergence of the BDF3 / quadratic ESFEM discretisation for MCF for a sphere

$\left[0, T_{\max }\right]=[0,0.6]$. For our computations we used a sequence of time step sizes $\tau_{k}=\tau_{k-1} / 2$ with $\tau_{0}=0.2$, and a sequence of initial meshes of mesh widths $h_{k} \approx 2^{-1 / 2} h_{k-1}$ with $h_{0} \approx 0.5$.

In Figure 13.1 and 13.2 we report the errors between the numerical and exact solution (both for the surface error and the error of the dynamic variables $\nu$ and $H$ ) until time $T=0.6$. The logarithmic plots show the $L^{\infty}\left(H^{1}\right)$ norm errors against the time step size $\tau$ in Figure 13.1, and against the mesh width $h$ in Figure 13.2. The lines marked with different symbols correspond to different mesh refinements and to different time step sizes in Figure 13.1 and 13.2, respectively.

In Figure 13.1 we can observe two regions: a region where the temporal discretisation error dominates, matching to the $O\left(\tau^{3}\right)$ order of convergence of our theoretical results, and a region, with small time step size, where the spatial discretization error dominates (the error curves flatten out). For Figure 13.2, the same description applies, but in the presented cases only the first region can be observed.

The convergence in time and in space as shown by Figures 13.1 and 13.2, respectively, is in agreement with the theoretical convergence results (note the reference lines).

13.2 Singular mean curvature flow

We consider the mean curvature flow starting from a dumbbell shaped surface $\Gamma^{0}$, given by the distance function

$$
d(x)=x_{1}^{2}+x_{2}^{2}+G\left(x_{3}^{2}\right)-0.04,
$$


with $G(s)=2 s(s-199 / 200)$. The evolution of such a surface is known to be singular, as it has often been reported in the literature before; see [42, Figure 3], [25, Figure 3.2], or [19, Figure 2], [26, Section 7] for numerical experiments.

In Figure 13.3 we compare the algorithm of Dziuk [19] (see (3.6)) and Algorithm (5.1) (with the two-step BDF method; starting values at $t_{1}$ are obtained by a linearly implicit backward Euler step) on a mesh with 10522 nodes and time step size $\tau \approx 3 \cdot 10^{-3}$. The figures show snapshots of the surface evolution at different times (from top to bottom). The left column shows plots from Dziuk's algorithm, the middle column from (5.1), while on the right we present the results of a normalized version of (5.1), where the normal vector $\nu_{h}$ computed from the discretized evolution equation is rescaled to unit norm at each node. Figure 13.5 shows the evolution and blow-up of the computed mean curvature.

We find that all three methods work reasonably well as long as the mean curvature flow stays away from the singularity, say for times $t \leq 0.07$ with the singularity near 0.08 . With all three methods, however, the distribution of the moving nodes on the surface appears most dense near the two widest horizontal circles of the dumbbell, which is not a desired behaviour. The methods behave differently shortly before the singularity (last row of pictures in Figure 13.3): Dziuk's method develops an instability, the method (5.1) remains stable but is past the pinch singularity too early, whereas the method (5.1) with normalized computed normal vectors behaves very well also close to the pinch singularity; see Figure 13.4. In the unnormalized method, the computed normal vector grows fast in norm shortly before the singularity, see Figure 13.6, which speeds up the evolution according to $v=-H \nu$. This effect is delayed when a finer time and space grid are used.

\section{Conclusion and further comments}

In this paper we have given the first proof of convergence of an evolving finite element method for mean curvature flow of a closed surface. Under sufficient regularity assumptions on the evolving surface we have proved optimal-order convergence. Unlike Dziuk's method, the numerical method proposed here relies on the discretization of evolution equations for geometric quantities, which are then used in the velocity law.

In the convergence analysis we have clearly separated the issues of consistency and stability. The geometry enters only in the consistency error bounds, but not in the stability analysis. The latter is done in the matrix-vector formulation of the method based on the technique of energy estimates - here by testing the error equation with the (discretized) time derivative of the error vector - and on results from [33] that relate the mass and stiffness matrices for different finite element surfaces. Together with inverse estimates, this allows us to control the $W^{1, \infty}$ norm of the discrete surface, which is essential in proving stability estimates that, together with consistency estimates, 

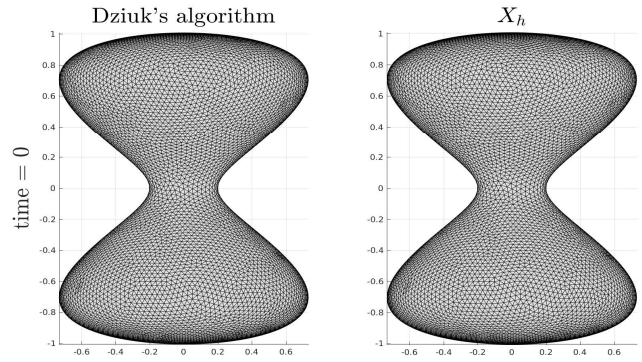

$X_{h}$ normalized
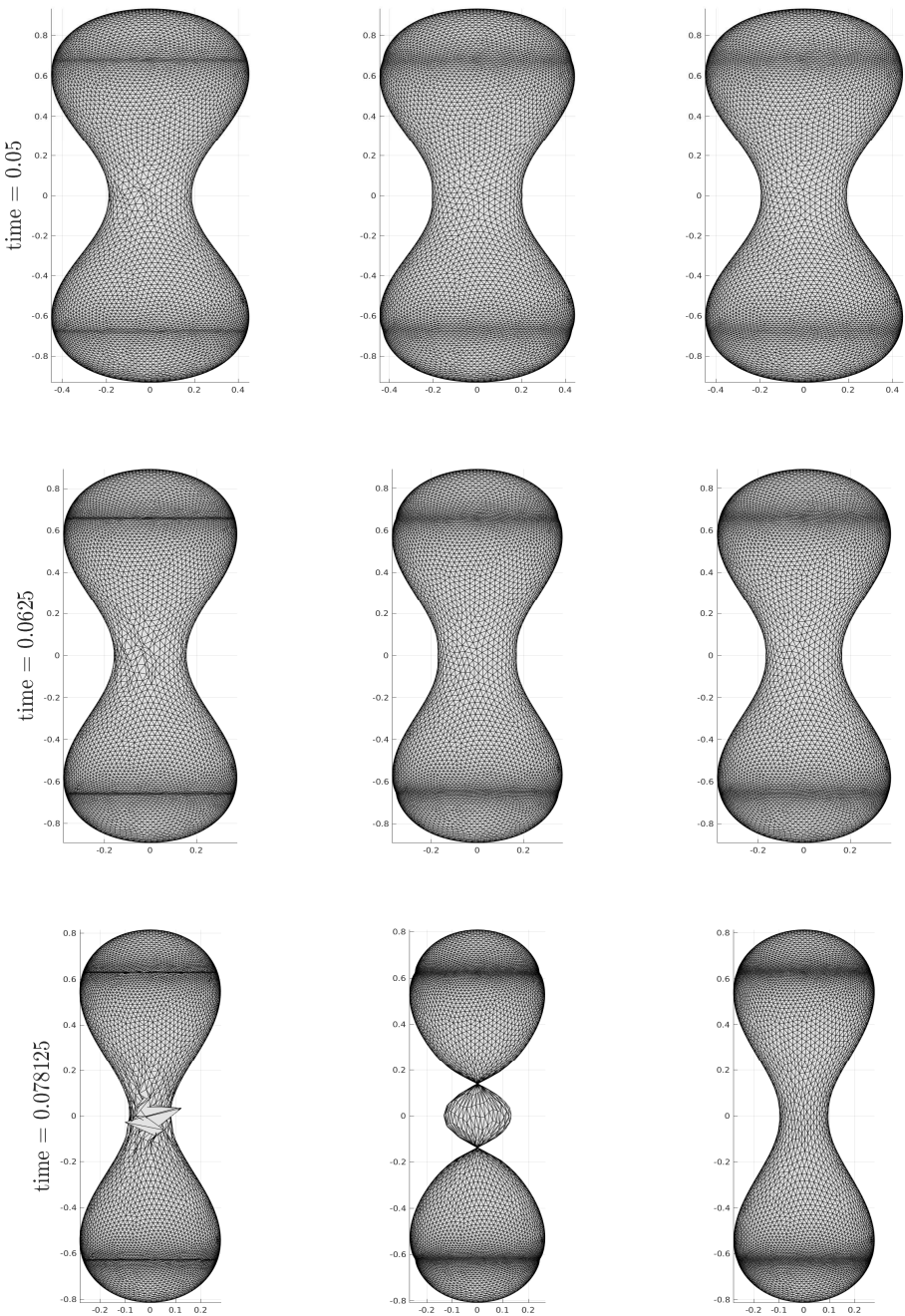

Fig. 13.3 Comparison of Dziuk's algorithm, of Algorithm (5.1), and of its version with normalized approximate normal vector $\nu_{h}$ on a flow developing a pinch singularity. 

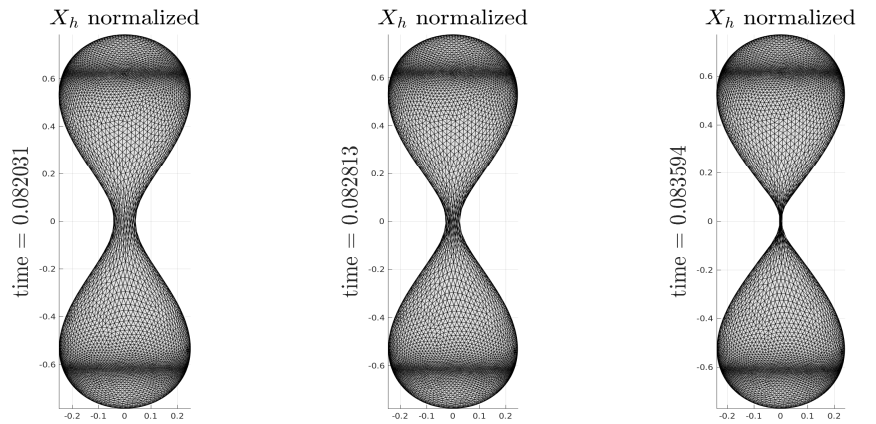

Fig. 13.4 The version of (5.1) with normalized $\nu_{h}$ close to the pinch singularity.
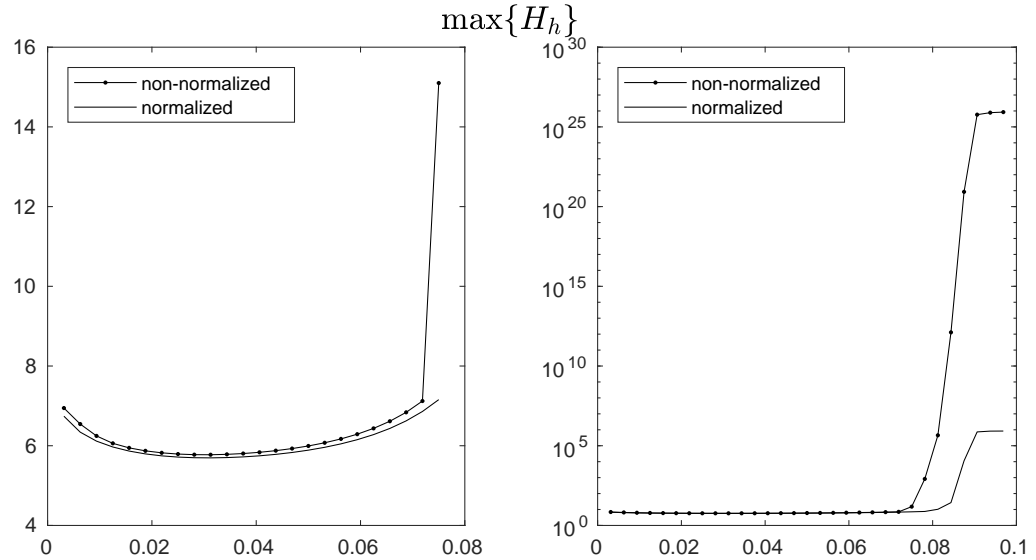

Fig. 13.5 Maximum of mean curvature and blow-up plotted against time.
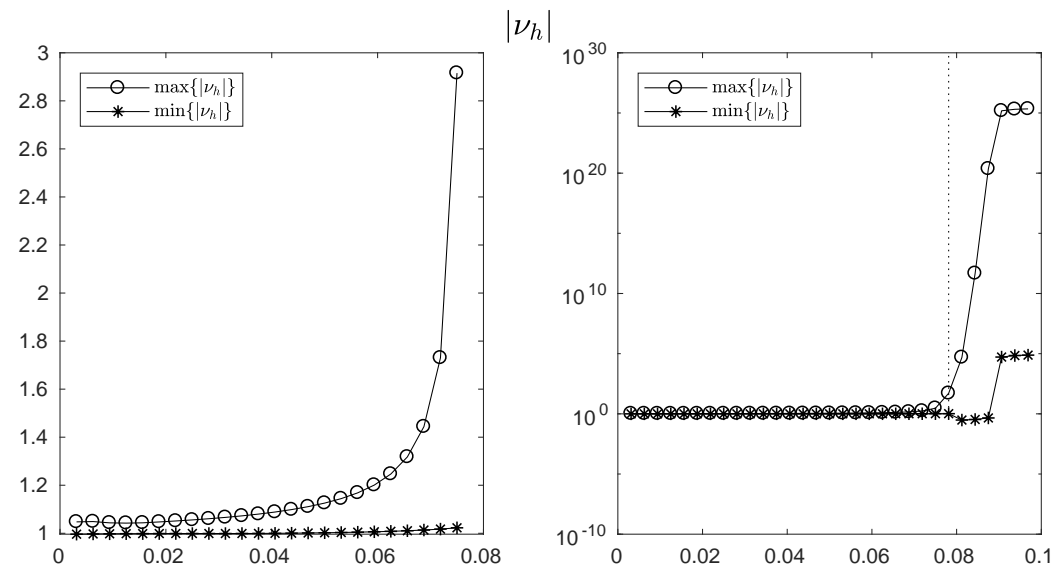

Fig. 13.6 Maximum and minimum length of normal vector and blow-up plotted against time. 
yield convergence. However, our stability analysis does not work for Dziuk's method, as is explained in Remark 7.1.

The numerical method studied here is based on the discretization of the evolution equations for the normal vector and the mean curvature, for which we then obtain optimal-order error bounds in the $H^{1}$ norm. Instead, one could also consider the evolution equations for other geometric quantities, such as the second fundamental form. When this two-form is expressed in local coordinates on each element, then a discontinuous Galerkin discretization appears as the method of choice to account for different local coordinates on different finite elements.

We expect that the discretization of evolution equations of geometric quantities will turn out useful also for the numerical treatment of other geometric evolution equations. For example, the extension of our numerical approach and its analysis appear fairly direct for mean curvature flow coupled to diffusion on the surface and also for inverse mean curvature flow, for which the corresponding evolution equations are given in [31].

On the practical side, given that we now have a convergent algorithm that provides optimal-order approximations also to the normal vector and to the mean curvature, is this the method that we would recommend for practical computations? Not quite. There are at least two aspects that need to be addressed to increase the robustness of the method beyond very fine meshes, in particular in near-singular situations:

- Mesh quality: Like Dziuk's method, also the method presented here often does not yield a satisfactory distribution of the nodes on the surface. It is to be expected that an arbitrary Lagrange - Euler (ALE) approach combined with the procedure of this paper can substantially mitigate this problem. In the literature this problem has been addressed, for example, by Elliott and Fritz [26] using the DeTurck trick, and by Barrett, Garcke and Nürnberg in a series of papers $[4,5,6,7,8]$.

- The normal vector $\nu_{h}$ obtained from the discretized evolution equation is not the same as the normal vector $\nu_{\Gamma_{h}[\mathbf{x}]}$ of the discrete surface. This poses no problem as long as they are close to each other (which asymptotically for sufficiently fine meshes they are by our convergence result), but leads to artifacts once they differ substantially. The situation can be improved by adding a stabilizing term in the discretization that works against a drift of the two normal vectors: on the right-hand side of (3.1b) one can add a stabilizing term $-\alpha \int_{\Gamma_{h}[\mathbf{x}]}\left(\nu_{h}-\nu_{\Gamma_{h}[\mathbf{x}]}\right) \cdot \varphi_{h}^{\nu}$ with a parameter $\alpha>0$.

Moreover, the normal vector computed from the discretized evolution equation is not of unit norm. While this appears easy to remedy in practice by rescaling to unit norm at every node (as is done in Section 13), the effect of such a pointwise rescaling is not easily understood theoretically. This problem is familiar also in numerical methods for harmonic map heat flow and equations for micromagnetism; see, e.g. [10,40].

We presented the numerical method for two-dimensional closed surfaces, but both the formulation and the convergence analysis of the numerical method 
can be extended to closed smooth hypersurfaces of arbitrary dimension. This is straightforward for three-dimensional hypersurfaces in $\mathbb{R}^{4}$, where the proof extends verbatim. The only change is in the use of the inverse inequality for finite element functions between the $W^{1, \infty}$ and $H^{1}$ norms, where a factor $h^{-3 / 2}$ instead of $h^{-1}$ appears. This extra factor $h^{-1 / 2}$, however, does not affect the course of the proof. In higher dimensions, the error bounds for interpolation need to be replaced by analogous error bounds for quasi-interpolation, and the inverse estimates depend on the dimension, yielding a factor $h^{-d / 2}$ in dimension $d$. As a consequence, the minimal polynomial degree $k$ required for our stability and convergence analysis becomes $k \geq\lfloor d / 2\rfloor+1$ for a $d$-dimensional hypersurface in $\mathbb{R}^{d+1}$, where $\lfloor d / 2\rfloor$ is the largest integer smaller than $d / 2$. Under the stepsize restriction $\tau \leq C_{0} h$, we then also need the temporal order $q \geq\lfloor d / 2\rfloor+1$.

\section{Acknowledgement}

We thank Frank Loose and Gerhard Wanner for helpful comments, and we also thank Jörg Nick for helpful discussions.

We thank two anonymous referees for their constructive comments on a previous version.

The work was partially supported by a grant from the Germany/Hong Kong Joint Research Scheme sponsored by the Research Grants Council of Hong Kong and the German Academic Exchange Service (G-PolyU502/16). The work of Balázs Kovács and Christian Lubich is supported by Deutsche Forschungsgemeinschaft, SFB 1173.

\section{References}

1. G. Akrivis, B. Li, and C. Lubich. Combining maximal regularity and energy estimates for time discretizations of quasilinear parabolic equations. Mathematics of Computation, 86(306):1527-1552, 2017.

2. G. Akrivis and C. Lubich. Fully implicit, linearly implicit and implicit-explicit backward difference formulae for quasi-linear parabolic equations. Numerische Mathematik, 131(4):713-735, 2015.

3. J. Barrett, K. Deckelnick, and V. Styles. Numerical analysis for a system coupling curve evolution to reaction diffusion on the curve. SIAM Journal on Numerical Analysis, 55(2):1080-1100, 2017.

4. J. Barrett, H. Garcke, and R. Nürnberg. On the variational approximation of combined second and fourth order geometric evolution equations. SIAM Journal on Scientific Computing, 29(3):1006-1041, 2007.

5. J. Barrett, H. Garcke, and R. Nürnberg. On the parametric finite element approximation of evolving hypersurfaces in $\mathbb{R}^{3}$. Journal of Computational Physics, 227(9):4281-4307, 2008.

6. J. Barrett, H. Garcke, and R. Nürnberg. Parametric approximation of Willmore flow and related geometric evolution equations. SIAM Journal on Scientific Computing, 31(1):225-253, 2008.

7. J. Barrett, H. Garcke, and R. Nürnberg. The approximation of planar curve evolutions by stable fully implicit finite element schemes that equidistribute. Numerical Methods for Partial Differential Equations, 27(1):1-30, 2011. 
8. J. Barrett, H. Garcke, and R. Nürnberg. Parametric approximation of isotropic and anisotropic elastic flow for closed and open curves. Numerische Mathematik, 120(3):489$542,2012$.

9. K. A. Brakke. The Motion of a Surface by Its Mean Curvature. Princeton University Press, 1978.

10. I. Cimrák. A survey on the numerics and computations for the Landau-Lifshitz equation of micromagnetism. Archives of Computational Methods in Engineering, 15(3):1-37, 2007.

11. G. Dahlquist. G-stability is equivalent to A-stability. BIT, 18:384-401, 1978.

12. K. Deckelnick. Error bounds for a difference scheme approximating viscosity solutions of mean curvature flow. Interfaces and Free Boundaries, 2(2):117-142, 2000.

13. K. Deckelnick and G. Dziuk. Convergence of a finite element method for non-parametric mean curvature flow. Numerische Mathematik, 72(2):197-222, 1995.

14. K. Deckelnick and G. Dziuk. On the approximation of the curve shortening flow. In Calculus of variations, applications and computations (Pont-à-Mousson, 1994), volume 326 of Pitman Res. Notes Math. Ser., pages 100-108. Longman Sci. Tech., Harlow, 1995.

15. K. Deckelnick and G. Dziuk. Error estimates for a semi-implicit fully discrete finite element scheme for the mean curvature flow of graphs. Interfaces and Free Boundaries, 2(4):341-359, 2000

16. K. Deckelnick, G. Dziuk, and C. M. Elliott. Computation of geometric partial differential equations and mean curvature flow. Acta Numerica, 14:139-232, 2005.

17. A. Demlow. Higher-order finite element methods and pointwise error estimates for elliptic problems on surfaces. SIAM Journal on Numerical Analysis, 47(2):805-807, 2009.

18. G. Dziuk. Finite elements for the Beltrami operator on arbitrary surfaces. Partial differential equations and calculus of variations, Lecture Notes in Math., 1357, Springer, Berlin, pages 142-155, 1988.

19. G. Dziuk. An algorithm for evolutionary surfaces. Numerische Mathematik, 58(1):603611,1990 .

20. G. Dziuk. Convergence of a semi-discrete scheme for the curve shortening flow. Mathematical Models and Methods in Applied Sciences, 4(04):589-606, 1994.

21. G. Dziuk and C. Elliott. Finite elements on evolving surfaces. IMA Journal of Numerical Analysis, 27(2):262-292, 2007.

22. G. Dziuk and C. Elliott. $L^{2}$-estimates for the evolving surface finite element method. Mathematics of Computation, 82(281):1-24, 2013.

23. G. Dziuk, D. Kröner, and T. Müller. Scalar conservation laws on moving hypersurfaces. Interfaces and Free Boundaries, 15(2):203-236, 2013.

24. G. Dziuk, C. Lubich, and D. Mansour. Runge-Kutta time discretization of parabolic differential equations on evolving surfaces. IMA Journal of Numerical Analysis, 32(2):394416, 2012.

25. K. Ecker. Regularity theory for mean curvature flow. Springer, 2012.

26. C. Elliott and H. Fritz. On approximations of the curve shortening flow and of the mean curvature flow based on the DeTurck trick. IMA Journal of Numerical Analysis, 37(2):543-603, 2017.

27. W. Gautschi. Numerical analysis. Birkhäuser Boston, Inc., Boston, MA, 1997. An introduction.

28. E. Hairer, S. P. Nø rsett, and G. Wanner. Solving ordinary differential equations. I, volume 8 of Springer Series in Computational Mathematics. Springer-Verlag, Berlin, second edition, 1993. Nonstiff problems.

29. E. Hairer and G. Wanner. Solving Ordinary Differential Equations II. Stiff and Differential-Algebraic Problems. Springer, Berlin, Second edition, 1996.

30. G. Huisken. Flow by mean curvature of convex surfaces into spheres. J. Differential Geometry, 20(1):237-266, 1984.

31. G. Huisken and A. Polden. Geometric evolution equations for hypersurfaces. In Calculus of variations and geometric evolution problems (Cetraro, 1996), volume 1713 of Lecture Notes in Math., pages 45-84. Springer, Berlin, 1999.

32. B. Kovács. High-order evolving surface finite element method for parabolic problems on evolving surfaces. IMA Journal of Numerical Analysis, 38(1):430-459, 2018. 
33. B. Kovács, B. Li, C. Lubich, and C. Power Guerra. Convergence of finite elements on an evolving surface driven by diffusion on the surface. Numerische Mathematik, 137(3):643-689, 2017.

34. B. Kovács and C. Lubich. Linearly implicit full discretization of surface evolution. Numerische Mathematik, 140(1):121-152, 2018

35. B. Kovács and C. Power Guerra. Error analysis for full discretizations of quasilinear parabolic problems on evolving surfaces. Numerical Methods for Partial Differential Equations, 32(4):1200-1231, 2016.

36. C. Lubich, D. Mansour, and C. Venkataraman. Backward difference time discretization of parabolic differential equations on evolving surfaces. IMA Journal of Numerical Analysis, 33(4):1365-1385, 2013.

37. C. Mantegazza. Lecture Notes on Mean Curvature Flow. Progress in Mathematics, Volume 290. Birkhäuser, Corrected Printing 2012.

38. O. Nevanlinna and F. Odeh. Multiplier techniques for linear multistep methods. $\mathrm{Nu}$ merical Functional Analysis and Optimization, 3(4):377-423, 1981.

39. P.-O. Persson and G. Strang. A simple mesh generator in MATLAB. SIAM Review, 46(2):329-345, 2004.

40. A. Prohl. Computational micromagnetism. Teubner, Stuttgart, 2001.

41. S. W. Walker. The shape of things: a practical guide to differential geometry and the shape derivative. SIAM, Philadelphia, 2015.

42. B. White. Evolution of curves and surfaces by mean curvature. In Proceedings of the International Congress of Mathematicians, Vol. I (Beijing, 2002), pages 525-538. Higher Ed. Press, Beijing, 2002. 